\title{
Stereocontrolled Synthesis and Cycloaddition of
}

\section{1,2,4-Trioxygenated-1,3-dienes}

Nicolas Pichon, ${ }^{a}$ Anne Harrison-Marchand, ${ }^{a}$ Loic Toupet ${ }^{b}$ and Jacques Maddaluno ${ }^{a, *}$

a'Laboratoire des Fonctions Azotées \& Oxygénées Complexes de l'IRCOF, UMR 6014 CNRS, Université de Rouen, 76821 Mont St Aignan,

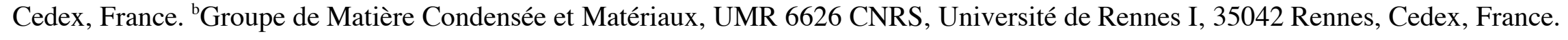

jmaddalu@crihan.fr

General experimental section: NMR analyses were conducted at room temperature at 500, 300 or $200 \mathrm{MHz}$ for ${ }^{1} \mathrm{H}$ NMR spectra and 125 , 75 or $50 \mathrm{MHz}$ for ${ }^{13} \mathrm{C}$ NMR spectra. Chemical shifts $(\delta)$ are given in ppm and the coupling constant $(J)$ in hertz. The solvent was deuteriochloroform, with a calibration at 7.26 for ${ }^{1} \mathrm{H}$ spectra and 77.36 for ${ }^{13} \mathrm{C}$ spectra. IR spectra were realized by transmission on a IRTF spectrometer. The mass spectra were obtained under electron impact conditions (EI) at $70 \mathrm{eV}$ ionizing potential; ammonia $\left(\mathrm{NH}_{3}\right)$ was used for chemical ionization (CI). The silica gel used for flash chromatography was $0.040-0.063 \mathrm{~mm}$. All reagents were of reagent grade and were used as such or distilled prior to use. 



\section{TABLE OF CONTENTS}

S1 : Title

S2-S5 : Table of contents

S6 : 1,1-Bis-(1-ethylpropyloxy)-propan-2-one $\mathbf{1 4 b}\left(300 \mathrm{MHz}, \mathrm{CDCl}_{3}\right)$

$\mathrm{S} 7$ : 1,1-Bis-(1-ethylpropyloxy)-propan-2-one $\mathbf{1 4 b}\left(75 \mathrm{MHz}, \mathrm{CDCl}_{3}\right)$

S8 : Cyclohexyl-(2,2-dimethoxy-1-methylethylidene)-amine $\mathbf{1 5 a}\left(200 \mathrm{MHz}, \mathrm{CDCl}_{3}\right)$

S9 : Cyclohexyl-(2,2-dimethoxy-1-methylethylidene)-amine $15 \mathbf{a}\left(75 \mathrm{MHz}, \mathrm{CDCl}_{3}\right)$

$\mathrm{S} 10$ : 2,2-Bis-(1-ethylpropyloxy)-1-methylethylidenecyclohexylamine $\mathbf{1 5 b}\left(200 \mathrm{MHz}, \mathrm{CDCl}_{3}\right)$

S11 : 2,2-Bis-(1-ethylpropyloxy)-1-methylethylidenecyclohexylamine $15 \mathbf{b}\left(50 \mathrm{MHz}, \mathrm{CDCl}_{3}\right)$

S12 : 1,1,4-Trimethoxybutan-2-one $18\left(300 \mathrm{MHz}, \mathrm{CDCl}_{3}\right)$

S13 : 1,1,4-Trimethoxybutan-2-one $18\left(75 \mathrm{MHz}, \mathrm{CDCl}_{3}\right)$

S14 : 4-Ethoxy-1,1-dimethoxybutan-2-one 19 (300 MHz, $\left.\mathrm{CDCl}_{3}\right)$

S15 : 4-Ethoxy-1,1-dimethoxybutan-2-one 19 (75 MHz, $\left.\mathrm{CDCl}_{3}\right)$

S16 : 4-Ethoxy-1,1-bis-(1-ethylpropyloxy)-butan-2-one 20 (300 MHz, $\mathrm{CDCl}_{3}$ )

$\mathrm{S} 17$ : 4-Ethoxy-1,1-bis-(1-ethylpropyloxy)-butan-2-one 20 (75 MHz, $\mathrm{CDCl}_{3}$ )

S18 : 1,1,4-Trimethoxy-2-triethylsilyloxybut-2-ene $\mathbf{1 2 a}\left(300 \mathrm{MHz}, \mathrm{CDCl}_{3}\right)$ 
S19 : 1,1,4-Trimethoxy-2-triethylsilyloxybut-2-ene 12a (75 MHz, $\mathrm{CDCl}_{3}$ )

S20 : 1,1,4-Trimethoxy-2-triisobutylsilyloxybut-2-ene 12b (300 MHz, $\left.\mathrm{CDCl}_{3}\right)$

S21 : 1,1,4-Trimethoxy-2-triisobutylsilyloxybut-2-ene $\mathbf{1 2 b}\left(75 \mathrm{MHz}, \mathrm{CDCl}_{3}\right)$

$\mathrm{S} 22$ : 2-(tert-Butyldiphenylsilyloxy)-1,1,4-trimethoxybut-2-ene $12 \mathrm{c}\left(300 \mathrm{MHz}, \mathrm{CDCl}_{3}\right)$

S23 : 2-(tert-Butyldiphenylsilyloxy)-1,1,4-trimethoxybut-2-ene $12 \mathrm{c}\left(75 \mathrm{MHz}, \mathrm{CDCl}_{3}\right)$

$\mathrm{S} 24$ : 4-Ethoxy-1,1-dimethoxy-2-triethylsilyloxybut-2-ene 12d (300 MHz, $\left.\mathrm{CDCl}_{3}\right)$

S25 : 4-Ethoxy-1,1-dimethoxy-2-triethylsilyloxybut-2-ene 12d $\left(75 \mathrm{MHz}, \mathrm{CDCl}_{3}\right)$

S26 : 2-(tert-Butyldimethylsilyloxy)-4-ethoxy-1,1-dimethoxybut-2-ene $\mathbf{1 2 e}\left(300 \mathrm{MHz}, \mathrm{CDCl}_{3}\right)$

S27 : 2-(tert-Butyldimethylsilyloxy)-4-ethoxy-1,1-dimethoxybut-2-ene $\mathbf{1 2 e}\left(75 \mathrm{MHz}, \mathrm{CDCl}_{3}\right)$

S28 : 4-Ethoxy-1,1-dimethoxy-2-triisopropylsilyloxybut-2-ene $12 f\left(300 \mathrm{MHz}, \mathrm{CDCl}_{3}\right)$

S29 : 4-Ethoxy-1,1-dimethoxy-2-triisopropylsilyloxybut-2-ene $12 f\left(75 \mathrm{MHz}, \mathrm{CDCl}_{3}\right)$

S30 : 2-(tert-Butyldiphenylsilyloxy)-4-ethoxy-1,1-dimethoxybut-2-ene $\mathbf{1 2 g}\left(300 \mathrm{MHz}, \mathrm{CDCl}_{3}\right)$

S31 : 2-(tert-Butyldiphenylsilyloxy)-4-ethoxy-1,1-dimethoxybut-2-ene $12 \mathrm{~g}\left(75 \mathrm{MHz}, \mathrm{CDCl}_{3}\right)$

S32 : 4-Ethoxy-1-[bis-(1-ethylpropyloxy)]-2-triethylsilyloxybut-2-ene $\mathbf{1 2 h}\left(200 \mathrm{MHz}, \mathrm{CDCl}_{3}\right)$

$\mathrm{S} 33$ : 4-Ethoxy-1-[bis-(1-ethylpropyloxy)]-2-triethylsilyloxybut-2-ene $\mathbf{1 2 h}\left(50 \mathrm{MHz}, \mathrm{CDCl}_{3}\right)$

S34 : 1,4-Dimethoxy-2-triethylsilyloxy-1,3-butadiene 13a (300 MHz, $\mathrm{CDCl}_{3}$ )

$\mathrm{S} 35$ : 1,4-Dimethoxy-2-triethylsilyloxy-1,3-butadiene 13a $\left(75 \mathrm{MHz}, \mathrm{CDCl}_{3}\right)$ 
S36 : 1,4-Dimethoxy-2-triisobutylsilyloxy-1,3-butadiene 13b (200 MHz, $\left.\mathrm{CDCl}_{3}\right)$

S37 : 1,4-Dimethoxy-2-triisobutylsilyloxy-1,3-butadiene 13b (50 MHz, $\left.\mathrm{CDCl}_{3}\right)$

S38 : 4-Ethoxy-1-methoxy-2-triethylsilyloxy-1,3-butadiene $\mathbf{1 3 d}\left(200 \mathrm{MHz}, \mathrm{CDCl}_{3}\right)$

S39 : 4-Ethoxy-1-methoxy-2-triethylsilyloxy-1,3-butadiene 13d (75 MHz, $\mathrm{CDCl}_{3}$ )

S40 : 2-tertButyldimethylsilyloxy-4-ethoxy-1-methoxy-1,3-butadiene 13e (300 $\left.\mathrm{MHz}, \mathrm{CDCl}_{3}\right)$

S41 : 2-tertButyldimethylsilyloxy-4-ethoxy-1-methoxy-1,3-butadiene 13e (75 MHz, $\mathrm{CDCl}_{3}$ )

$\mathrm{S} 42$ : 4-Ethoxy-1-methoxy-2-triisopropylsilyloxy-1,3-butadiene $13 f\left(200 \mathrm{MHz}, \mathrm{CDCl}_{3}\right)$

$\mathrm{S} 43$ : 4-Ethoxy-1-methoxy-2-triisopropylsilyloxy-1,3-butadiene $13 f\left(50 \mathrm{MHz}, \mathrm{CDCl}_{3}\right.$ )

$\mathrm{S} 44$ : 2-tertButyldiphenylsilyloxy-4-ethoxy-1-methoxy-1,3-butadiene $\mathbf{1 3 g}\left(300 \mathrm{MHz}, \mathrm{CDCl}_{3}\right.$ )

S45 : 2-tertButyldiphenylsilyloxy-4-ethoxy-1-methoxy-1,3-butadiene $13 g\left(75 \mathrm{MHz}, \mathrm{CDCl}_{3}\right.$ )

S46 : 4-Ethoxy-1-(1-ethylpropyloxy)-2-triethylsilyloxy-1,3-butadiene $13 \mathbf{h}$ (300 $\mathrm{MHz}, \mathrm{CDCl}_{3}$ )

$\mathrm{S} 47$ : 4-Ethoxy-1-(1-ethylpropyloxy)-2-triethylsilyloxy-1,3-butadiene $\mathbf{1 3 h}\left(75 \mathrm{MHz}, \mathrm{CDCl}_{3}\right)$

S48: 7-Ethoxy-4-methoxy-2-methyl-5-triethylsilyloxy-3a,4,7,7a-tetrahydroisoindole-1,3-dione 28 trans $\left(300 \mathrm{MHz}^{\mathrm{CDCl}}\right.$ )

S49 : 7-Ethoxy-4-methoxy-2-methyl-5-triethylsilyloxy-3a,4,7,7a-tetrahydroisoindole-1,3-dione 28 trans $\left(75 \mathrm{MHz}^{\mathrm{CDCl}}{ }_{3}\right)$

S50 : 7-Ethoxy-4-methoxy-2-methyl-5-triethylsilyloxy-3a,4,7,7a-tetrahydroisoindole-1,3-dione 28 cis (300 $\left.\mathrm{MHz} \mathrm{CDCl}_{3}\right)$

S51 : 7-Ethoxy-4-methoxy-2-methyl-5-triethylsilyloxy-3a,4,7,7a-tetrahydroisoindole-1,3-dione 28 cis $\left(75 \mathrm{MHz}^{\mathrm{CDCl}} \mathrm{CD}_{3}\right.$ 
S52 : 2-Ethoxy-5-methoxy-4-triethylsilyloxycyclohex-3-enecarboxylic acid phenyl ester 29 trans $\left(300 \mathrm{MHz} \mathrm{CDCl}_{3}\right)$

S53 : 2-Ethoxy-5-methoxy-4-triethylsilyloxycyclohex-3-enecarboxylic acid phenyl ester 29 trans (75 $\left.\mathrm{MHz} \mathrm{CDCl}_{3}\right)$

S54 : 2-Ethoxy-5-methoxy-4-triethylsilyloxycyclohex-3-enecarboxylic acid phenyl ester 29 cis (300 $\left.\mathrm{MHz}_{2} \mathrm{CDCl}_{3}\right)$

S55 : 2-Ethoxy-5-methoxy-4-triethylsilyloxycyclohex-3-enecarboxylic acid phenyl ester 29 cis $\left(75 \mathrm{MHz} \mathrm{CDCl}_{3}\right)$

S56 : 5-tertButyldiphenylsilyloxy-7-ethoxy-4-methoxy-2-methyl-3a,4,7,7a-tetrahydroisoindole-1,3-dione 30 trans (300 $\left.\mathrm{MHz}^{\mathrm{CDCl}}{ }_{3}\right)$

S57 : 5-tertButyldiphenylsilyloxy-7-ethoxy-4-methoxy-2-methyl-3a,4,7,7a-tetrahydroisoindole-1,3-dione 30 trans $\left(75 \mathrm{MHz}^{\mathrm{CDCl}} \mathrm{CH}_{3}\right.$

S58 : 5-tertButyldiphenylsilyloxy-7-ethoxy-4-methoxy-2-methyl-3a,4,7,7a-tetrahydroisoindole-1,3-dione 30 cis (300 MHz, CDCl $)$

S59 : 5-tertButyldiphenylsilyloxy-7-ethoxy-4-methoxy-2-methyl-3a,4,7,7a-tetrahydroisoindole-1,3-dione 30 cis (75 MHz, CDCl 3 )

S60 : 4-tert-Butyldiphenylsilyloxy-2,5-dimethoxycyclohex-3-enecarboxylic acid phenyl ester 31 trans $\left(300 \mathrm{MHz} \mathrm{CDCl}_{3}\right)$

S61 : 4-tert-Butyldiphenylsilyloxy-2,5-dimethoxycyclohex-3-enecarboxylic acid phenyl ester 31 trans $\left(75 \mathrm{MHz} \mathrm{CDCl}_{3}\right)$ 
1,1-Bis-(1-ethylpropyloxy)-propan-2-one 14b

(300 MHz, $\mathrm{CDCl}_{3}$ )
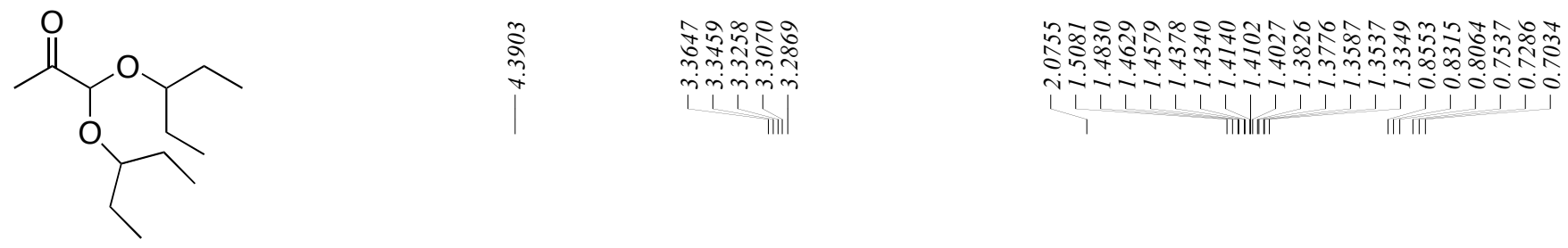

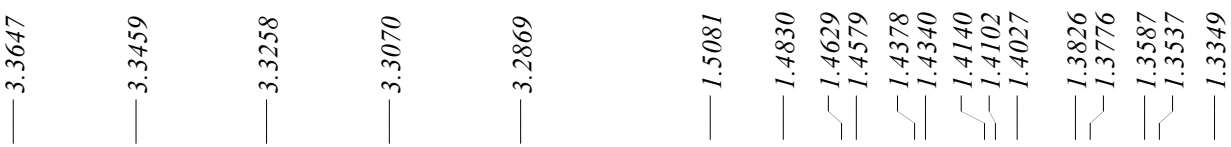
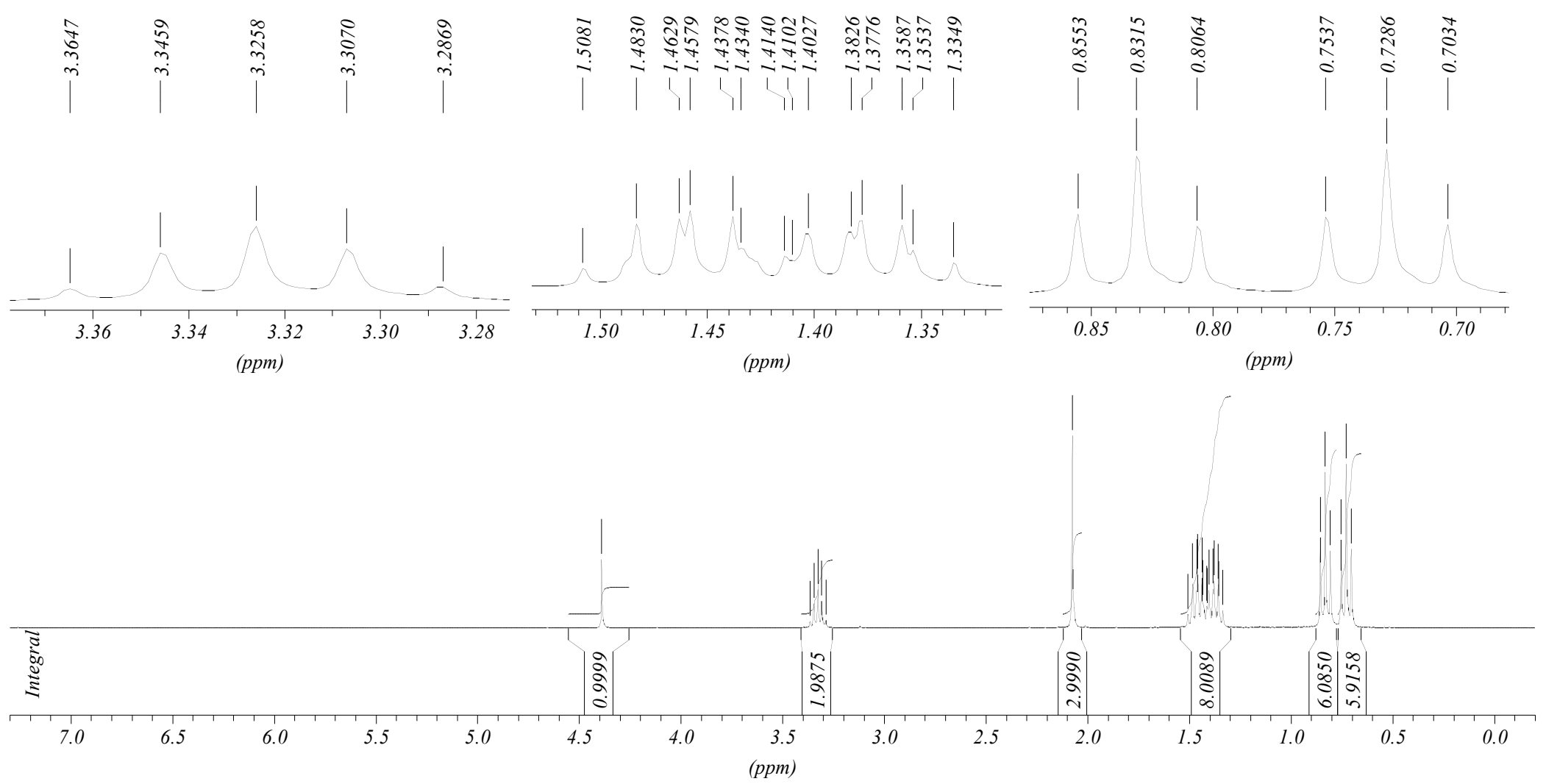
1,1-Bis-(1-ethylpropyloxy)-propan-2-one 14b

$\left(75 \mathrm{MHz}, \mathrm{CDCl}_{3}\right)$
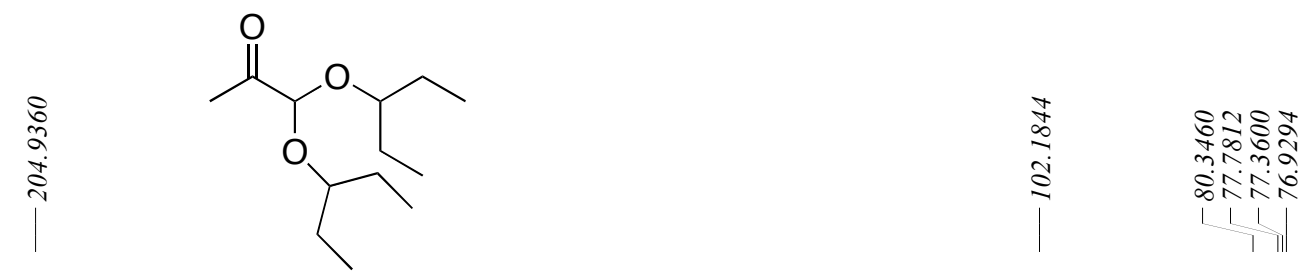

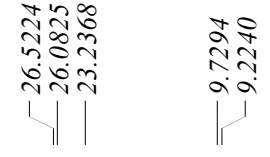

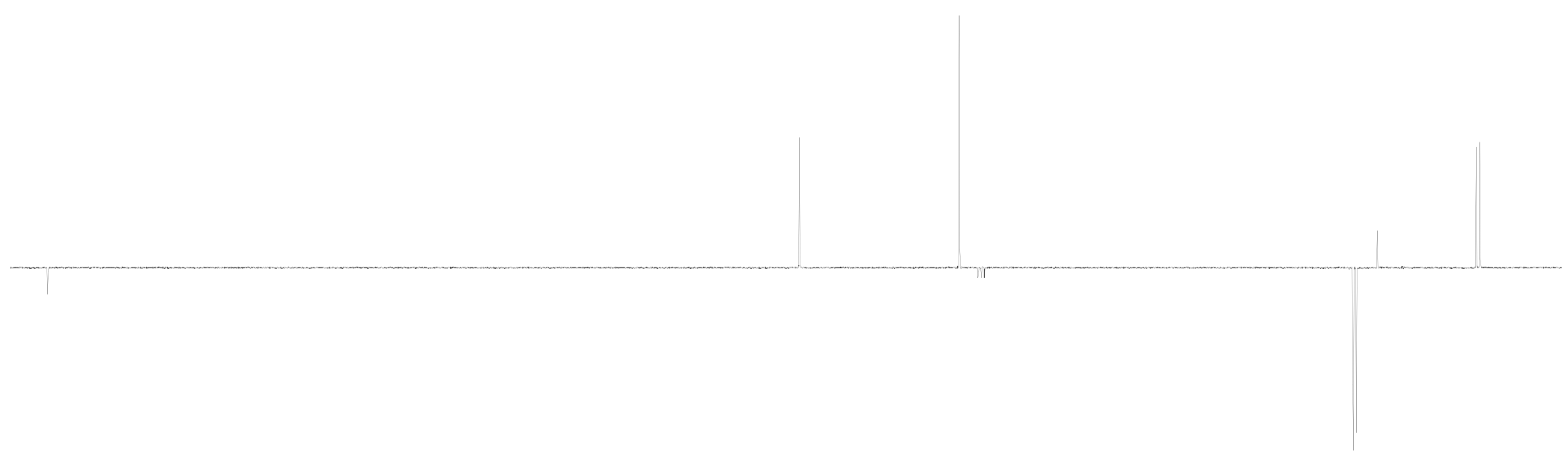




\section{Cyclohexyl-(2,2-dimethoxy-1-methylethylidene)-amine 15a}

$\left(200 \mathrm{MHz}, \mathrm{CDCl}_{3}\right)$

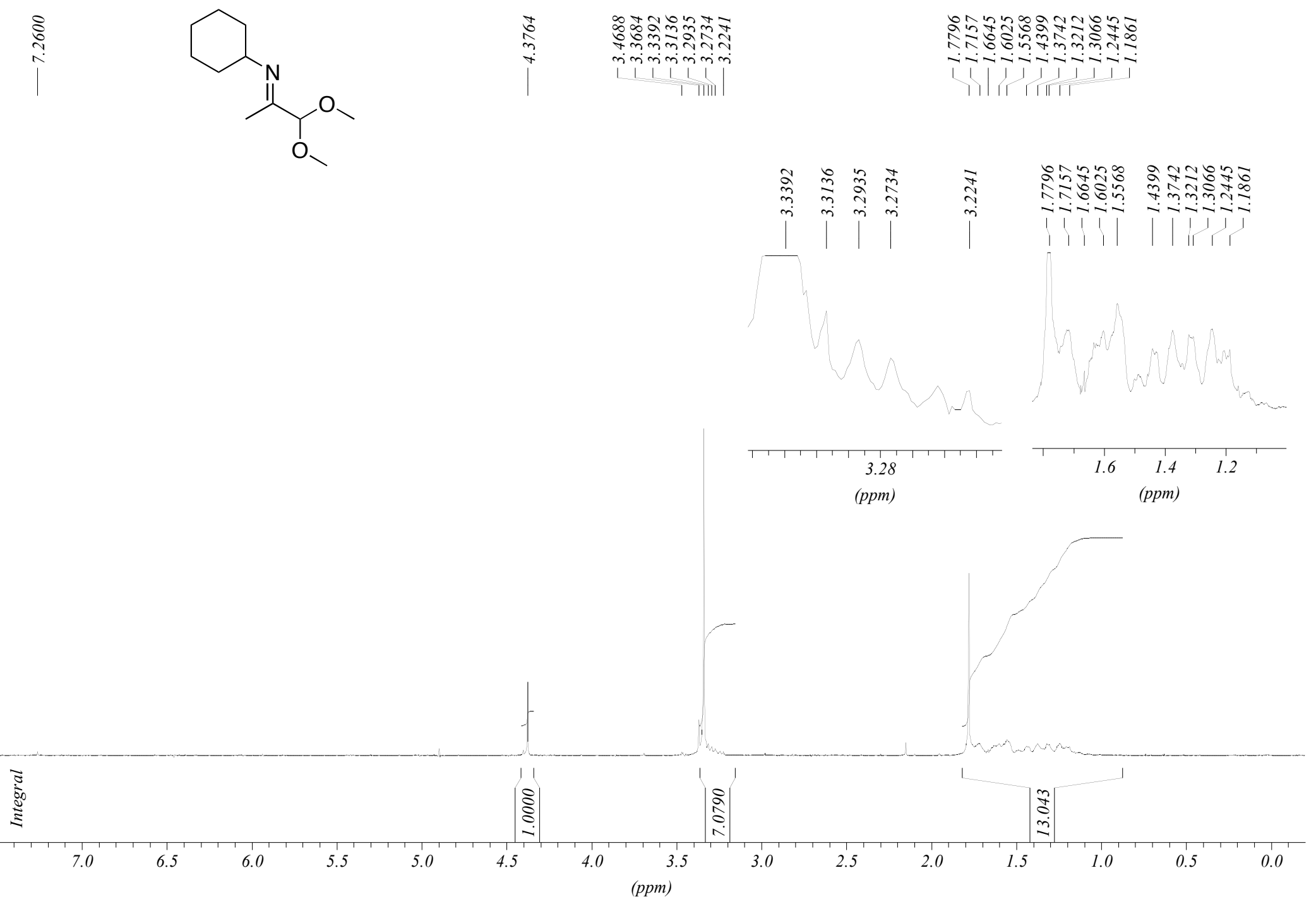


Cyclohexyl-(2,2-dimethoxy-1-methylethylidene)-amine 15a

$\left(75 \mathrm{MHz}, \mathrm{CDCl}_{3}\right)$
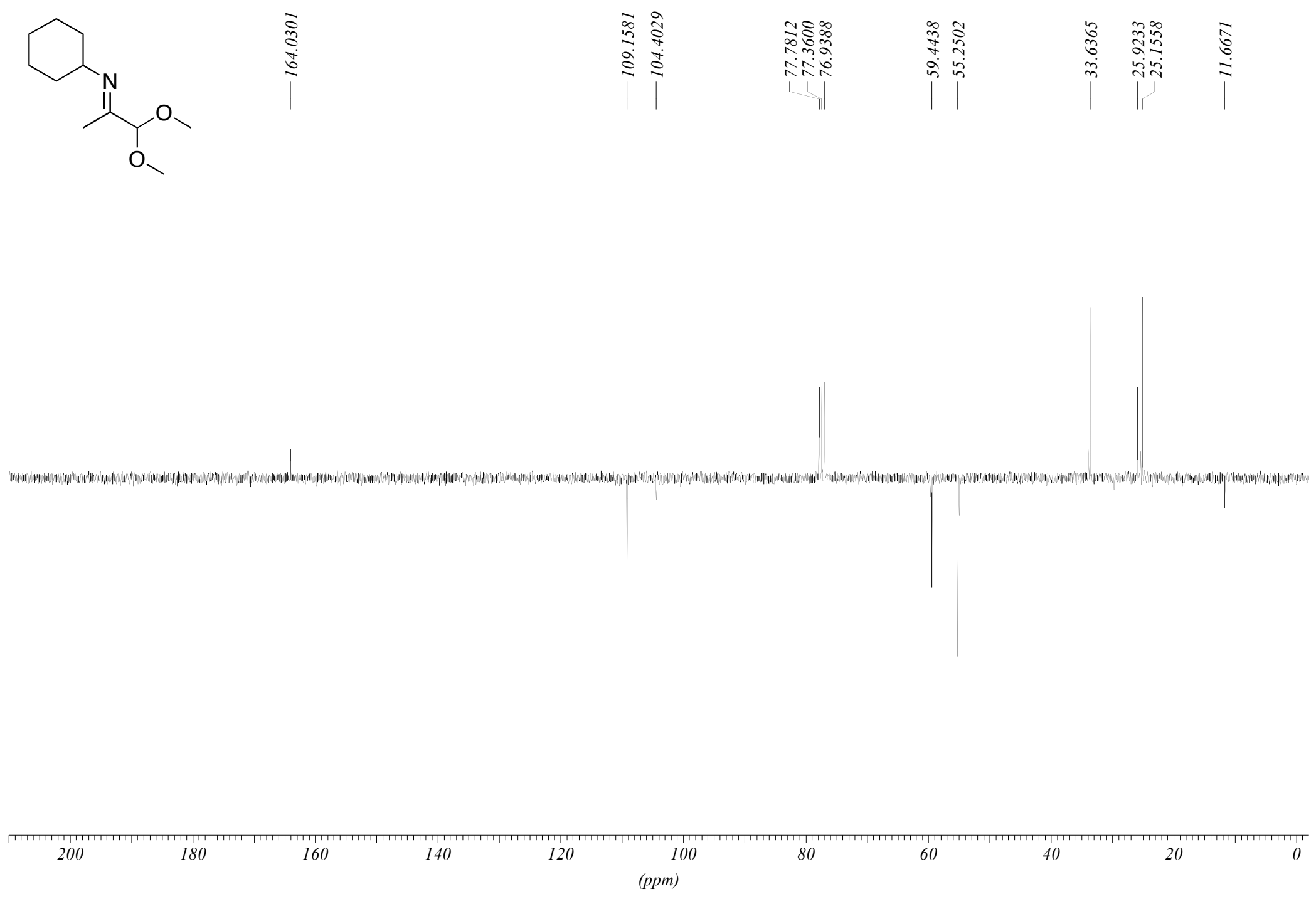
2,2-Bis-(1-ethylpropyloxy)-1-methylethylidenecyclohexylamine $\mathbf{1 5 b}$

$\left(200 \mathrm{MHz}, \mathrm{CDCl}_{3}\right)$
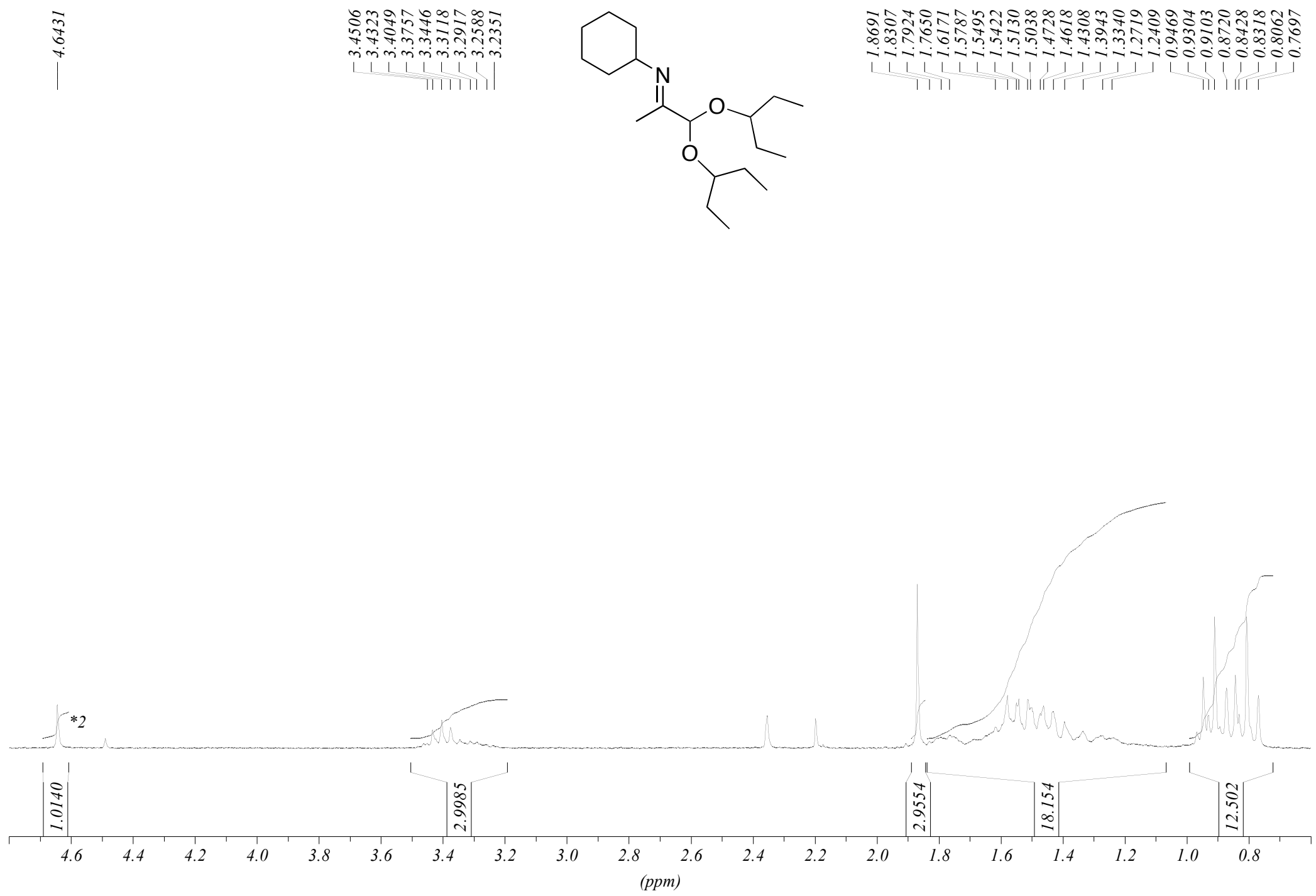
2,2-Bis-(1-ethylpropyloxy)-1-methylethylidenecyclohexylamine $\mathbf{1 5 b}$

$\left(50 \mathrm{MHz}, \mathrm{CDCl}_{3}\right)$

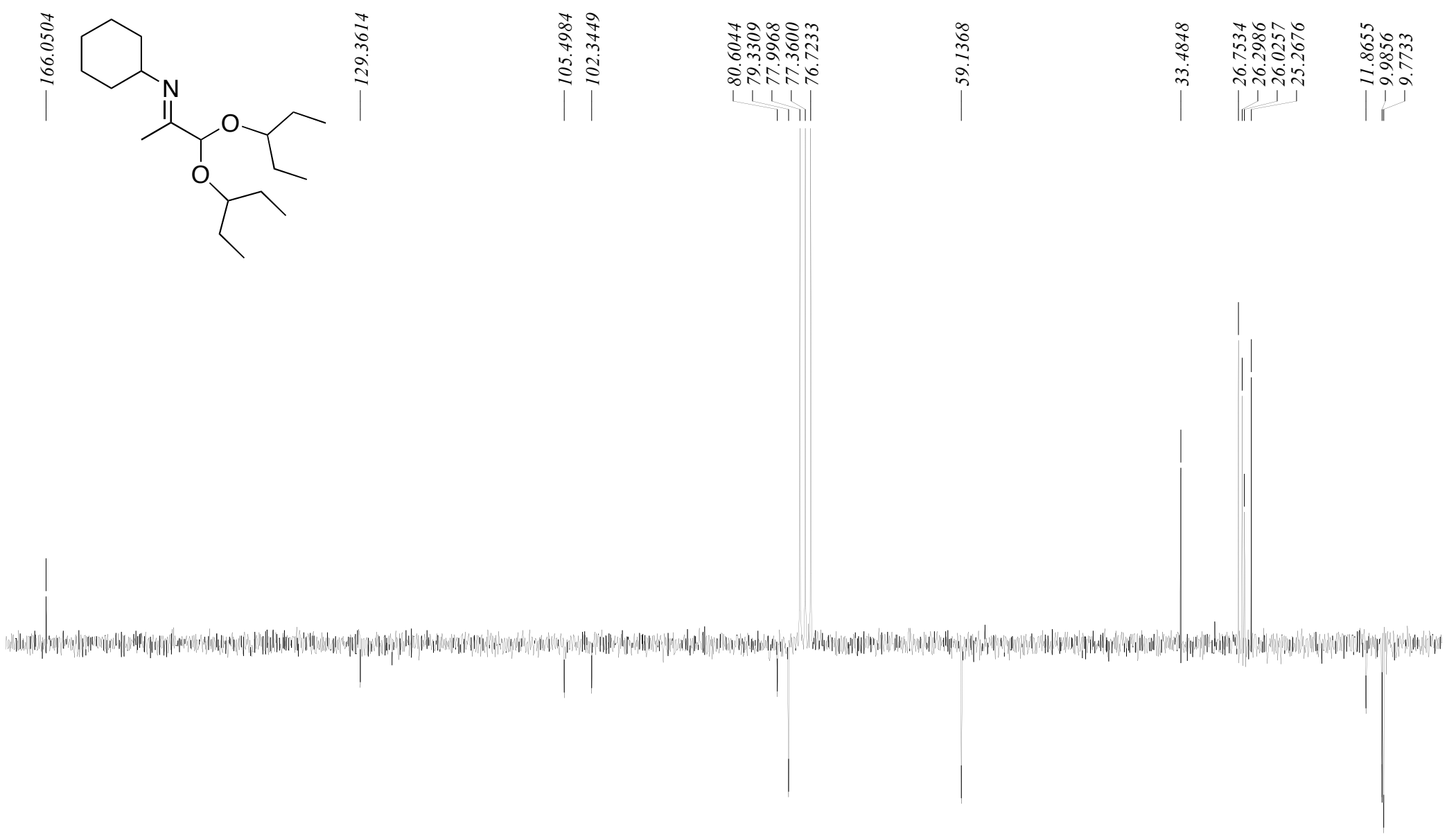

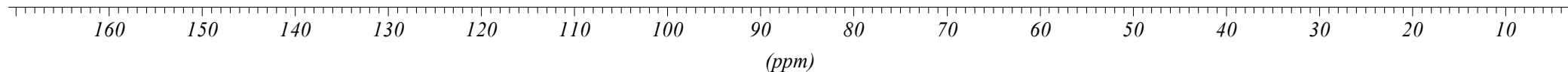


1,1,4-Trimethoxybutan-2-one $\mathbf{1 8}$

(300 MHz, $\mathrm{CDCl}_{3}$ )
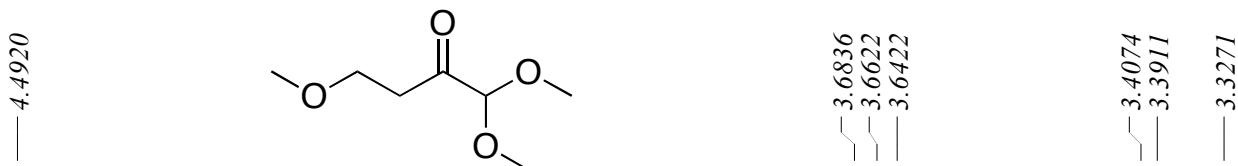

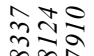

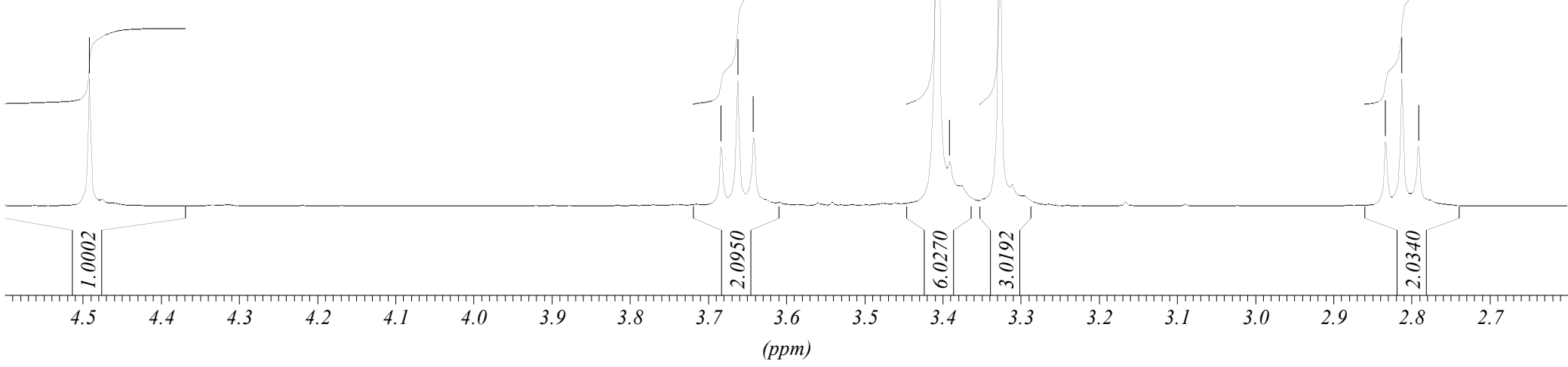


1,1,4-Trimethoxybutan-2-one $\mathbf{1 8}$

(75 MHz, $\mathrm{CDCl}_{3}$ )

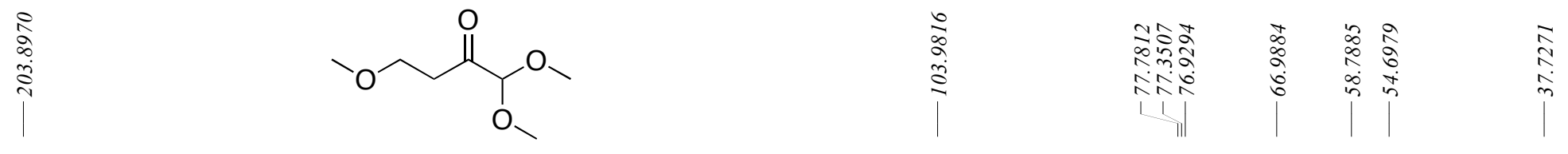

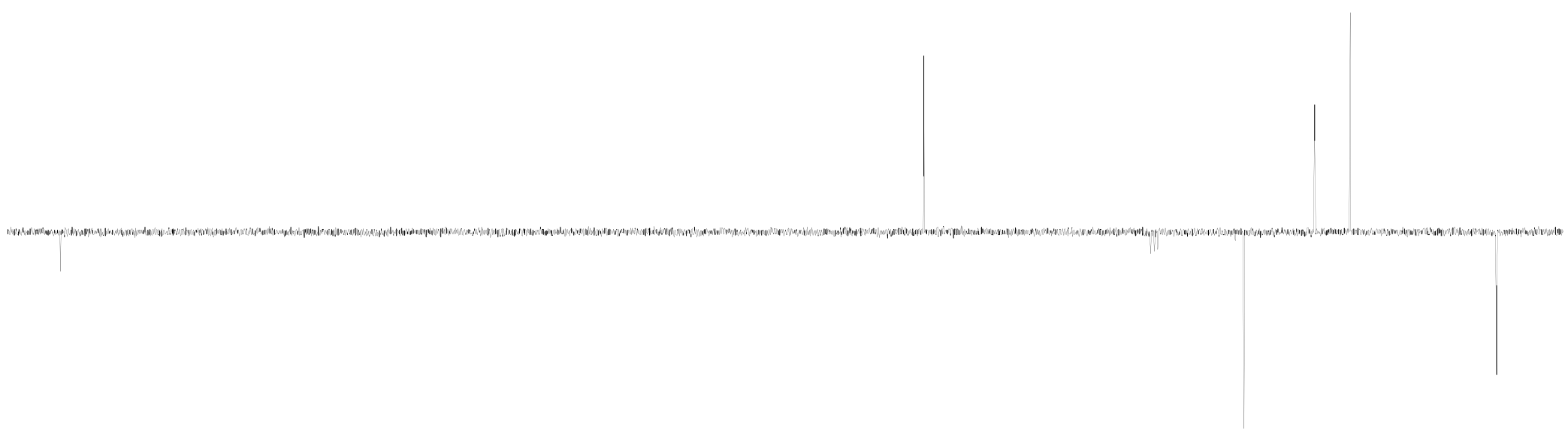

$170 \quad 160$

150

140

130

120

$110 \quad 100$

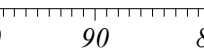

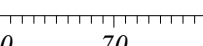


4-Ethoxy-1,1-dimethoxybutan-2-one 19 (300 MHz, $\mathrm{CDCl}_{3}$ )
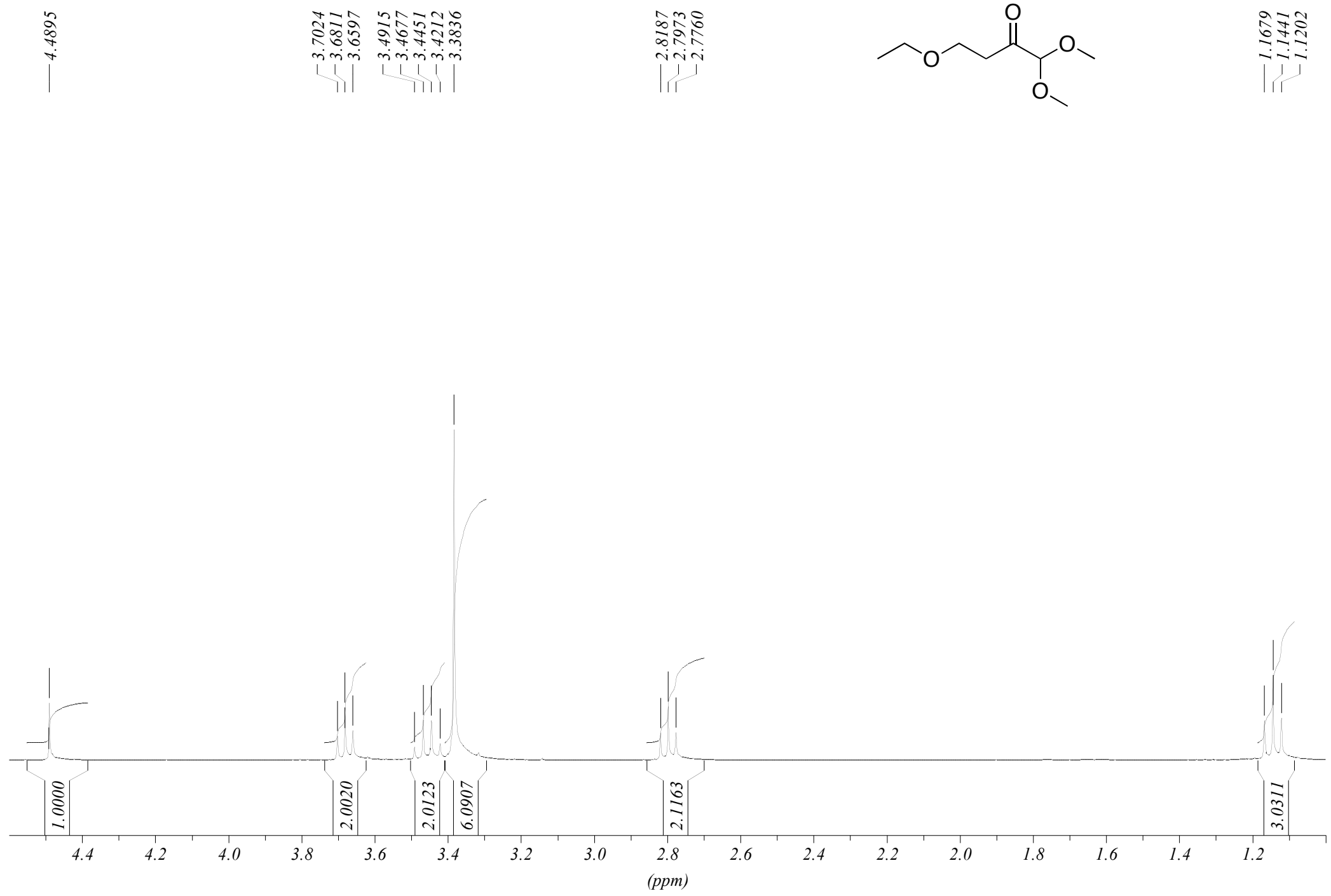
4-Ethoxy-1,1-dimethoxybutan-2-one 19

(75 MHz, $\mathrm{CDCl}_{3}$ )

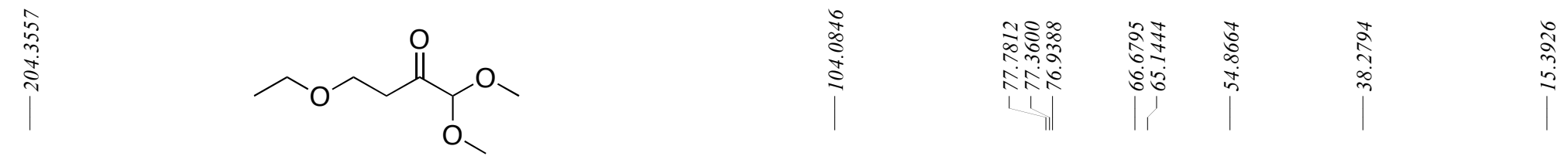

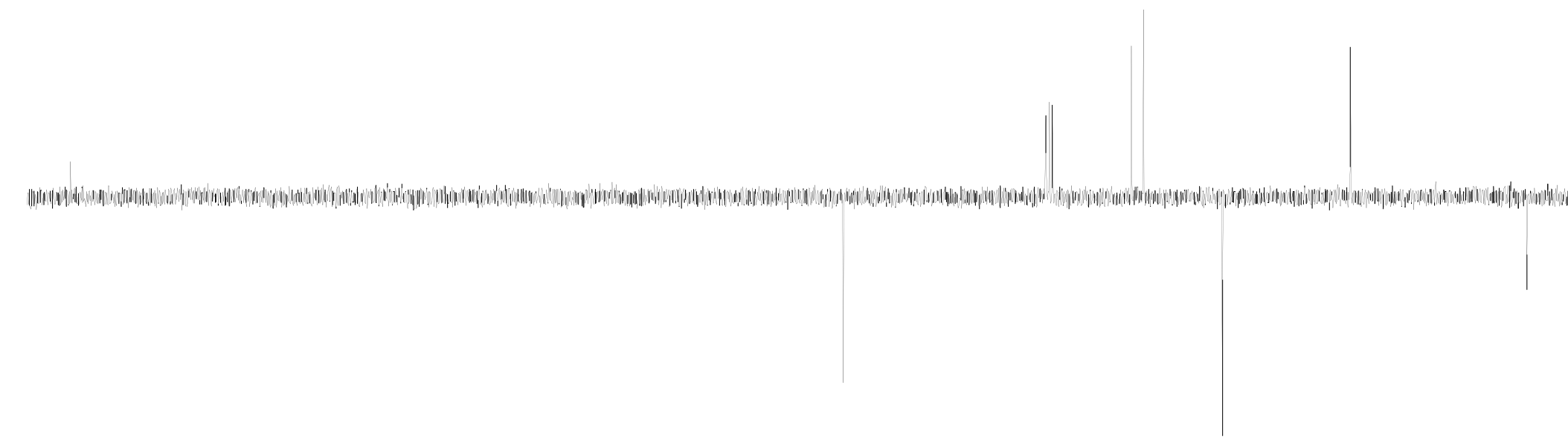

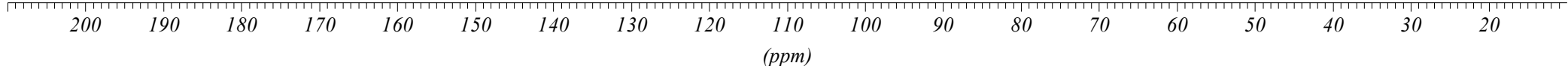


4-Ethoxy-1,1-bis-(1-ethylpropyloxy)-butan-2-one 20

(300 MHz, $\mathrm{CDCl}_{3}$ )
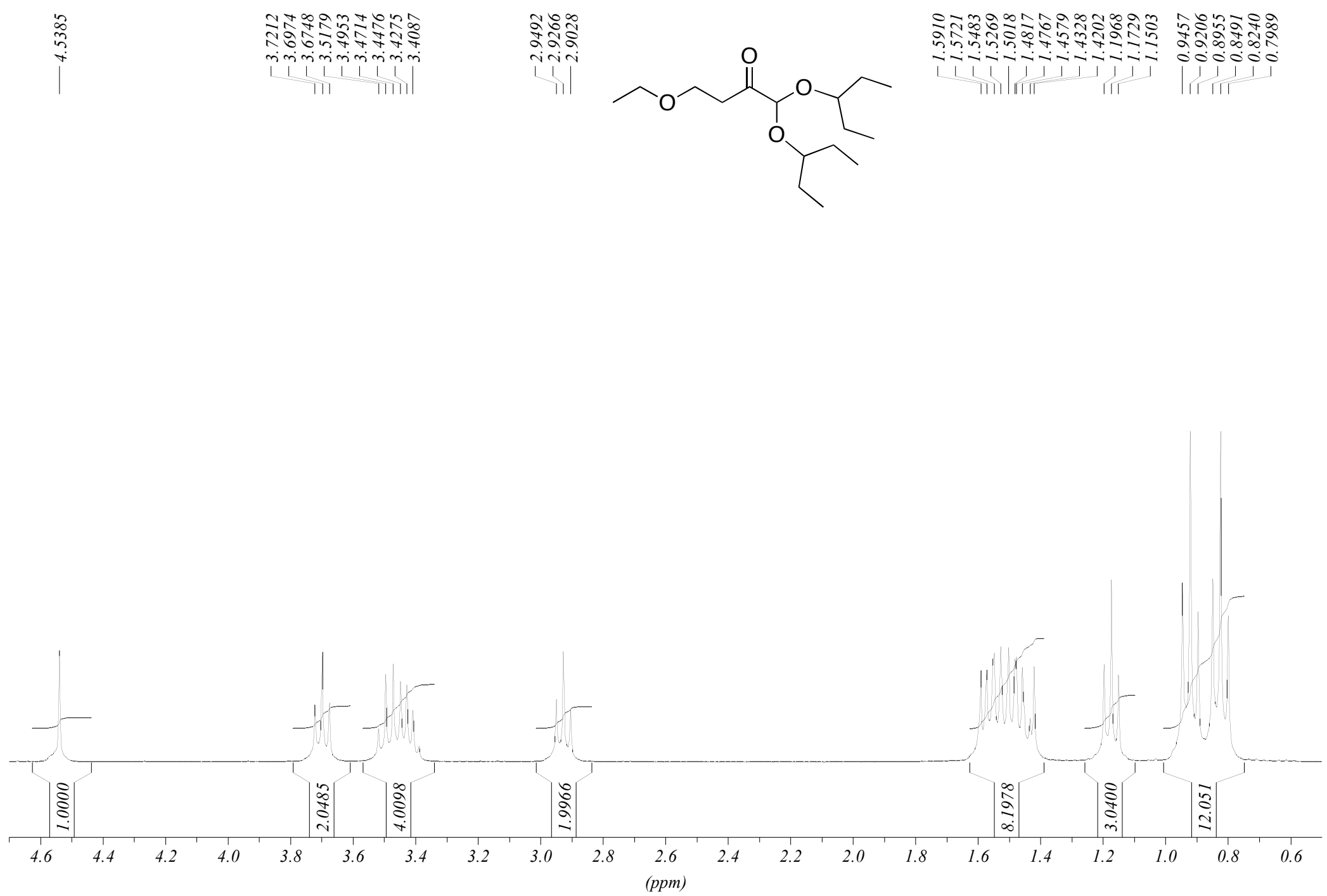
4-Ethoxy-1,1-bis-(1-ethylpropyloxy)-butan-2-one 20

(75 $\mathrm{MHz}, \mathrm{CDCl}_{3}$ )
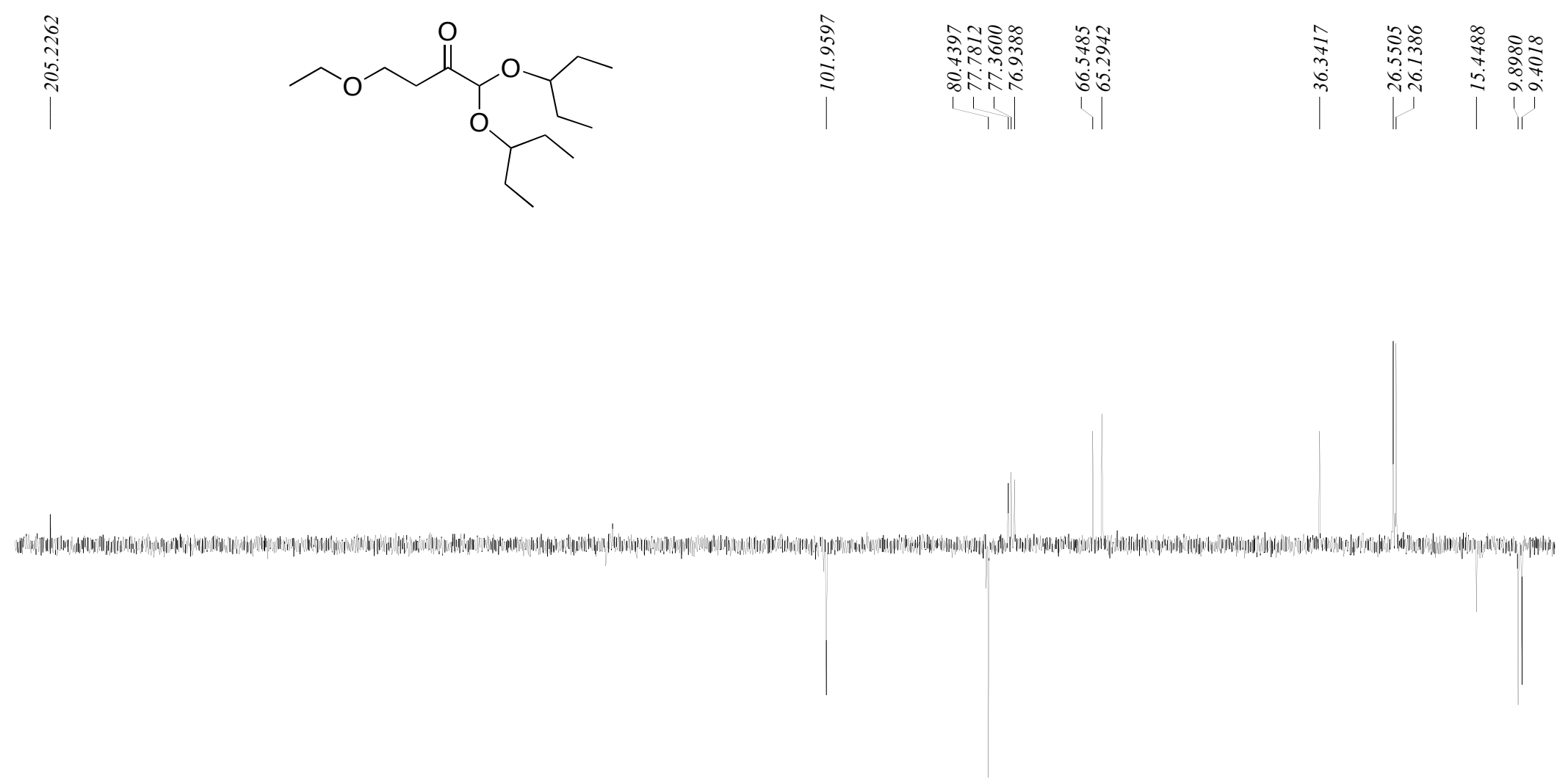

$170 \quad 160 \quad 150$

$130 \quad 120$ 
1,1,4-Trimethoxy-2-triethylsilyloxybut-2-ene 12a

(300 MHz, $\mathrm{CDCl}_{3}$ )
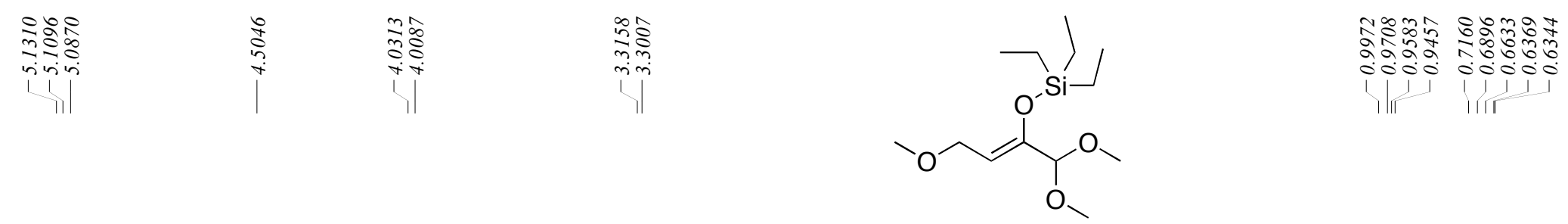

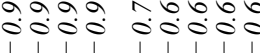
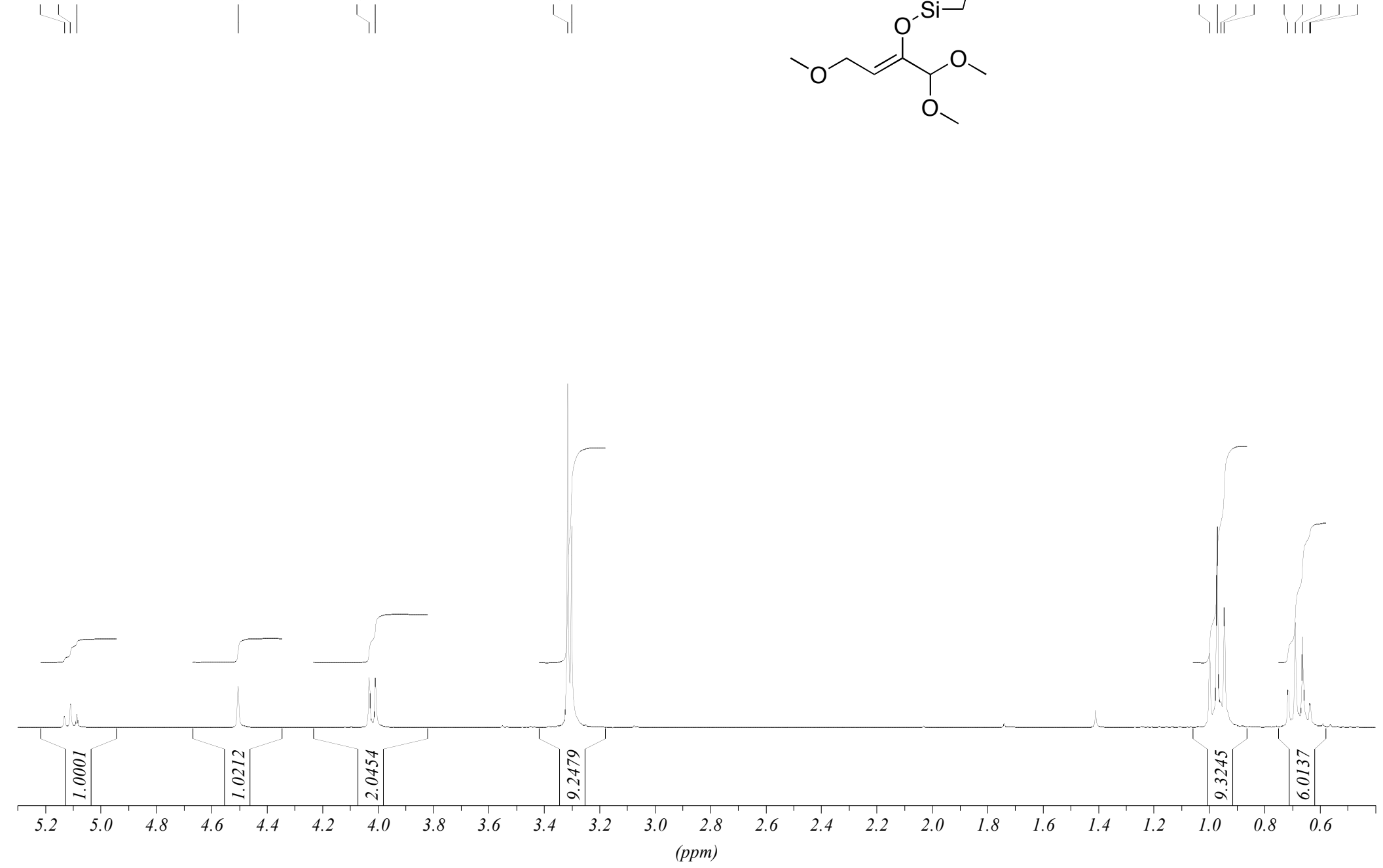
1,1,4-Trimethoxy-2-triethylsilyloxybut-2-ene 12a

(75 MHz, $\mathrm{CDCl}_{3}$ )

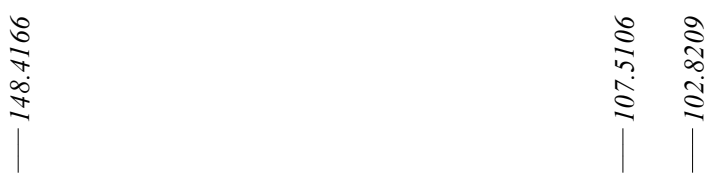

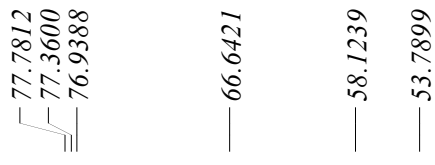
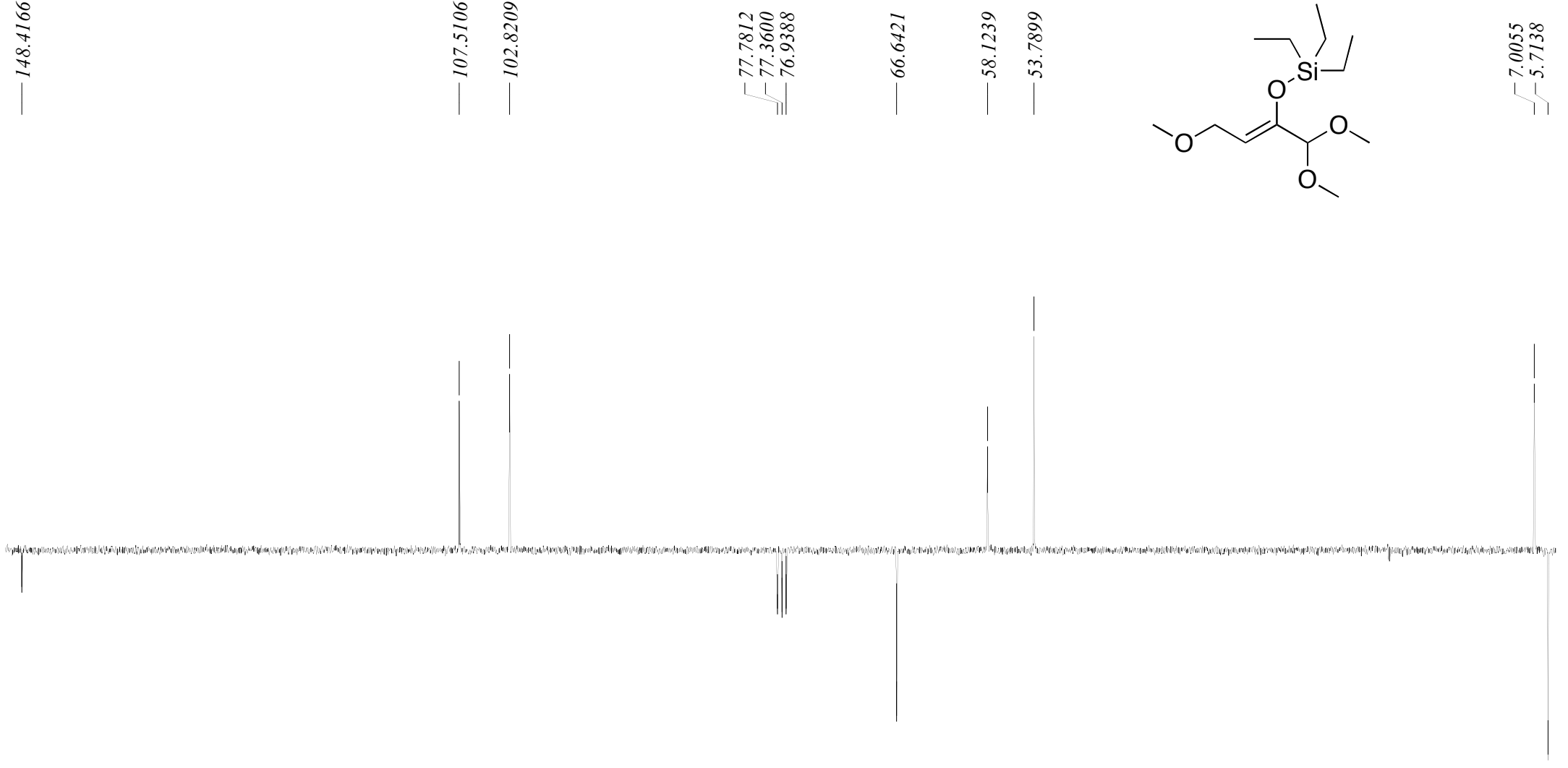

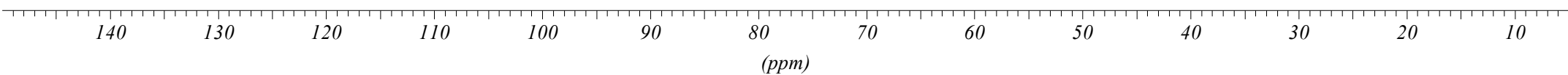


1,1,4-Trimethoxy-2-triisobutylsilyloxybut-2-ene $\mathbf{1 2 b}$

(300 MHz, $\mathrm{CDCl}_{3}$ )
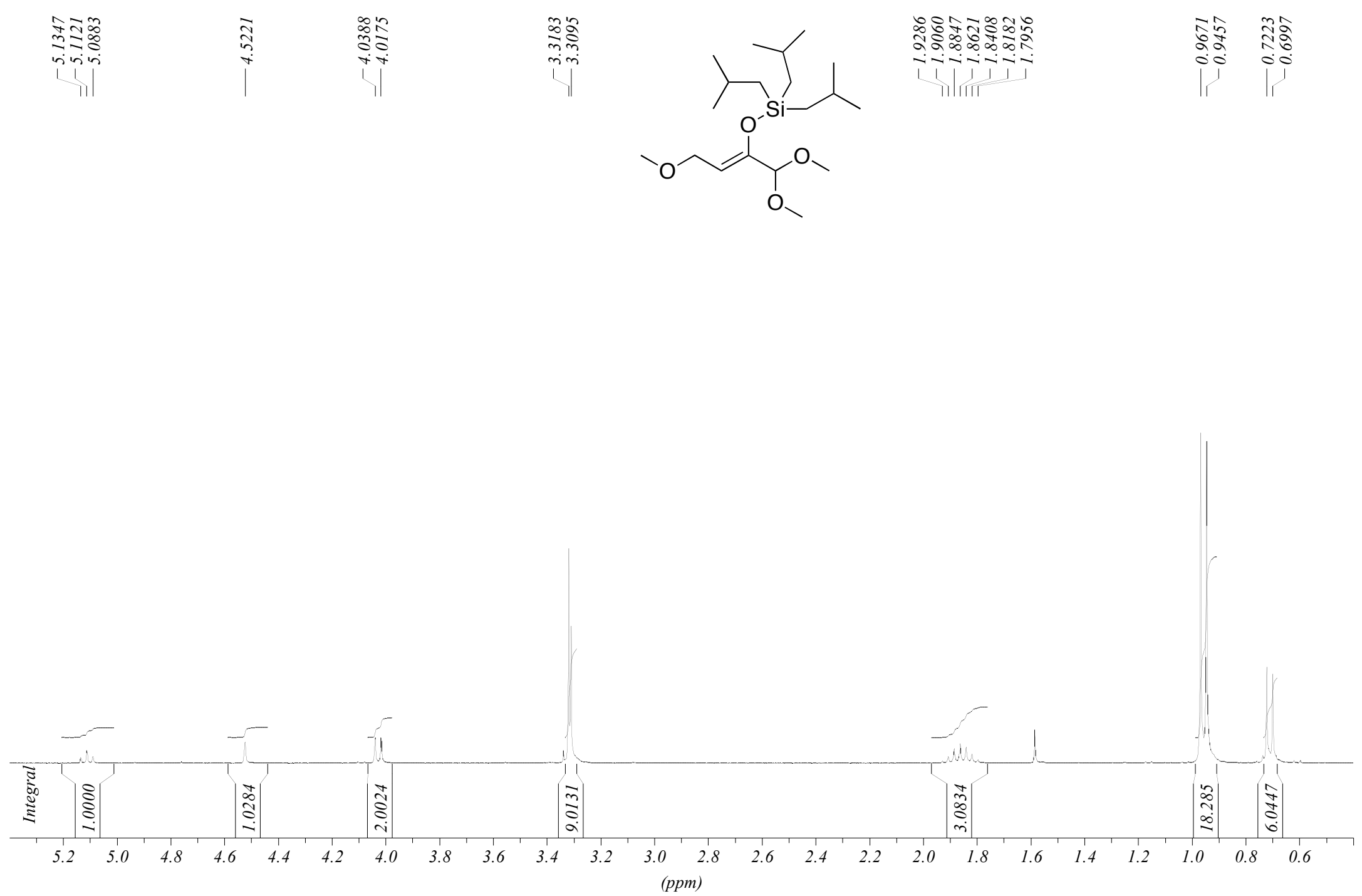
1,1,4-Trimethoxy-2-triisobutylsilyloxybut-2-ene $\mathbf{1 2 b}$

(75 MHz, $\mathrm{CDCl}_{3}$ )
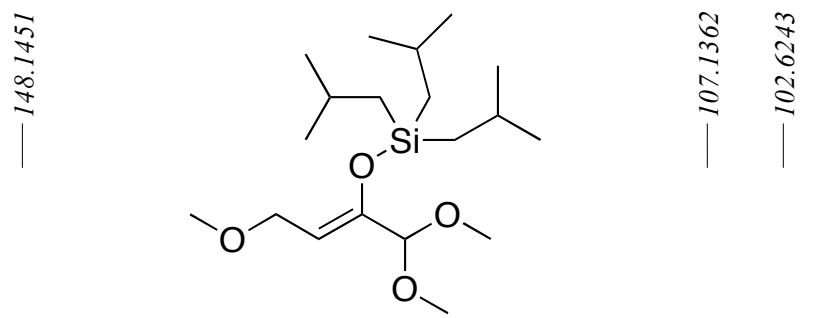

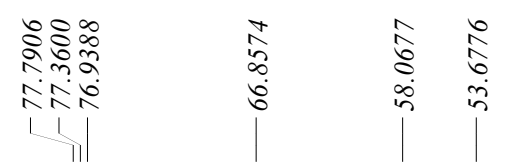
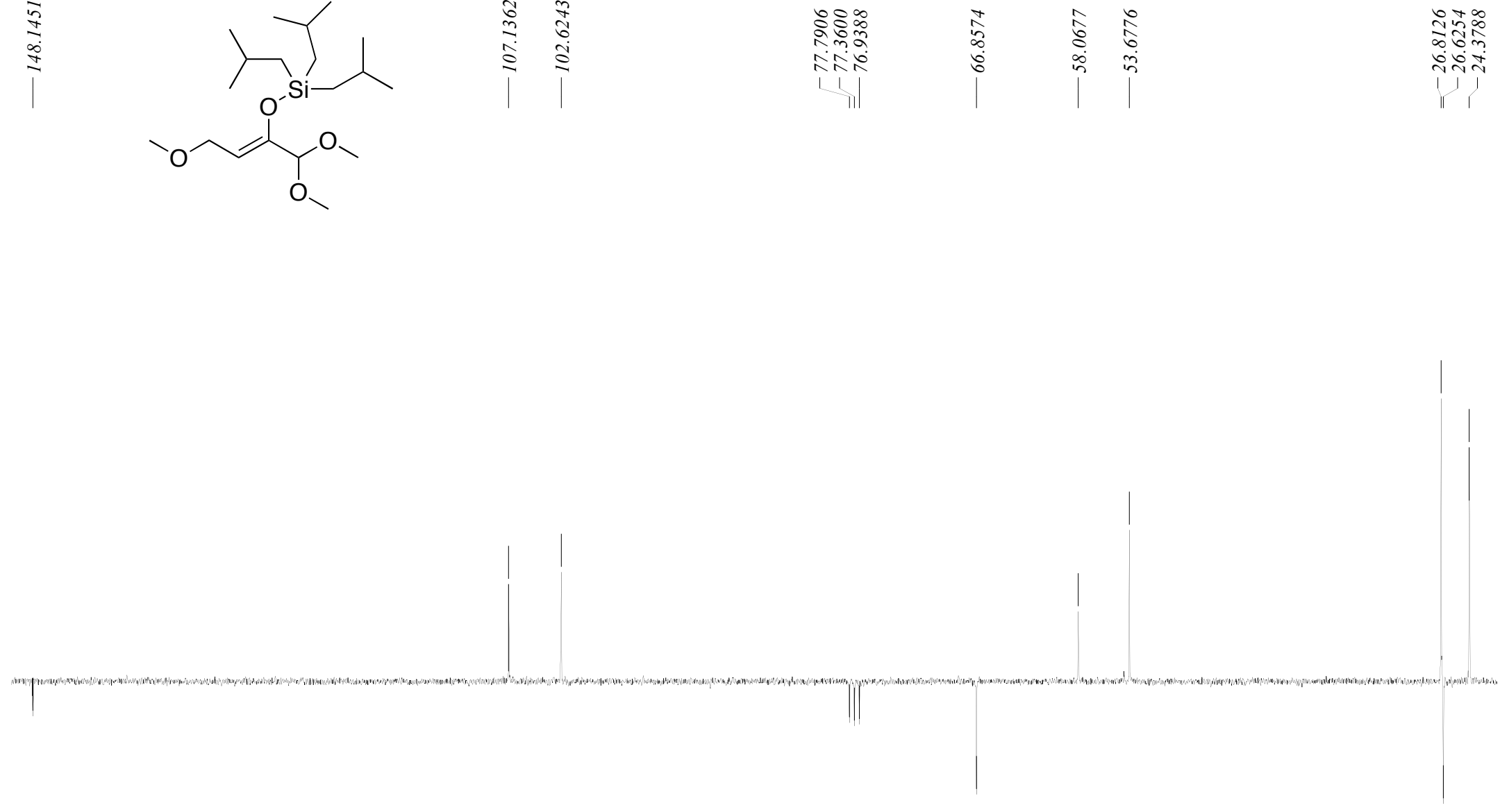

$\begin{array}{lllllllllllllll}145 & 140 & 135 & 130 & 125 & 120 & 115 & 110 & 105 & 100 & 95 & 90 & 85\end{array}$ (ppm) 
2-(tert-Butyldiphenylsilyloxy)-1,1,4-trimethoxybut-2-ene 12c $\left(300 \mathrm{MHz}, \mathrm{CDCl}_{3}\right)$
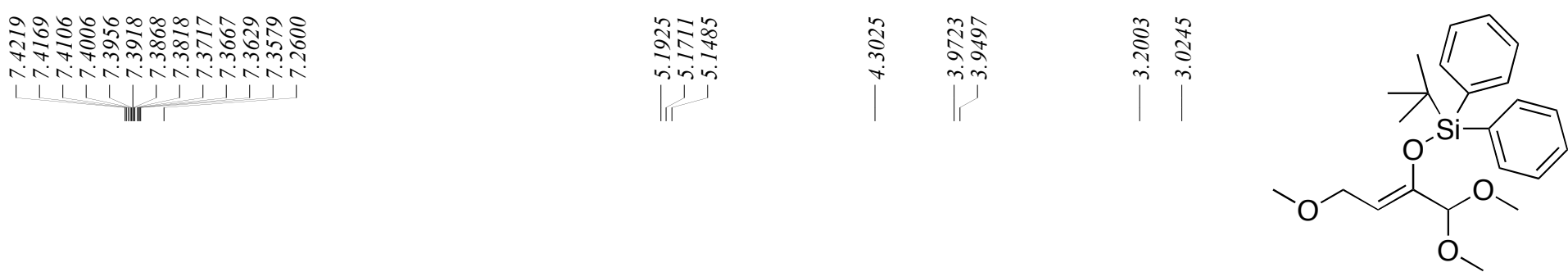

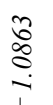

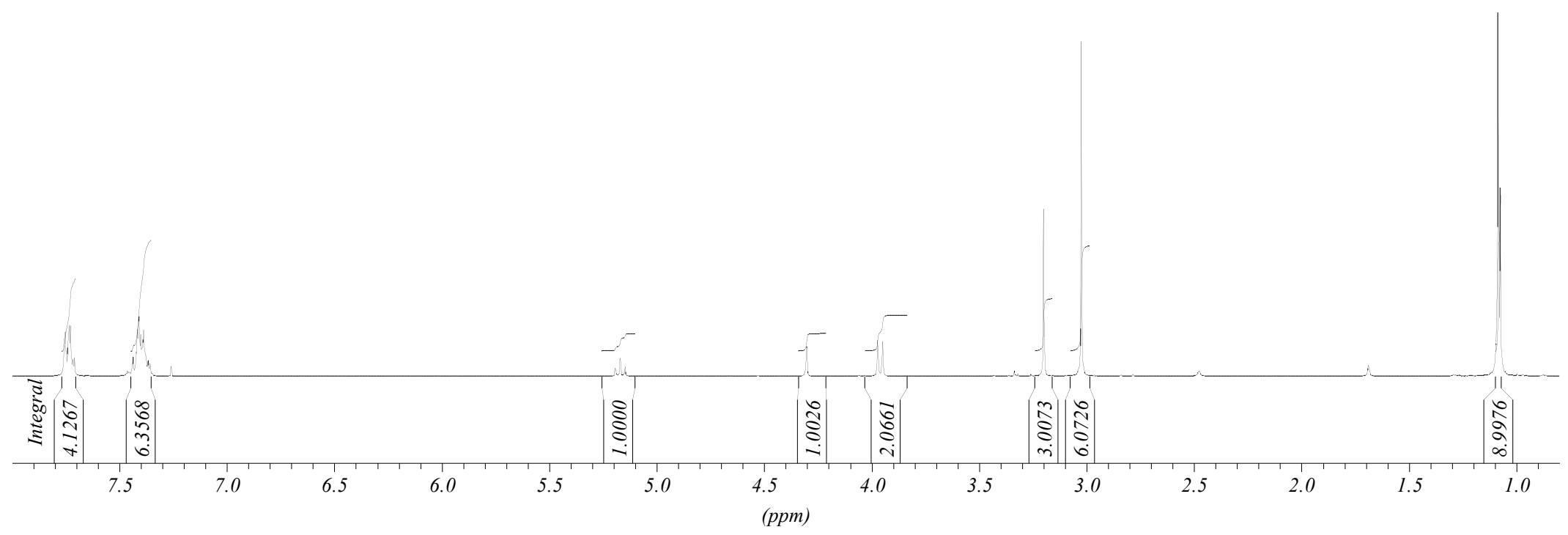


2-(tert-Butyldiphenylsilyloxy)-1,1,4-trimethoxybut-2-ene 12c $\left(75 \mathrm{MHz}, \mathrm{CDCl}_{3}\right)$

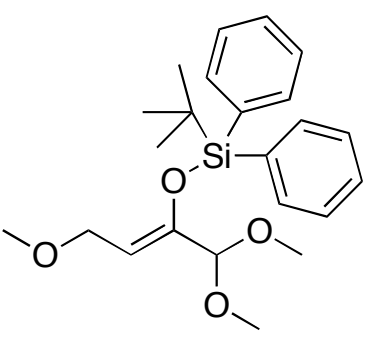

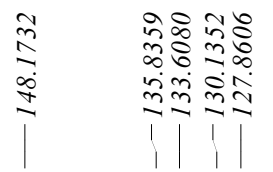

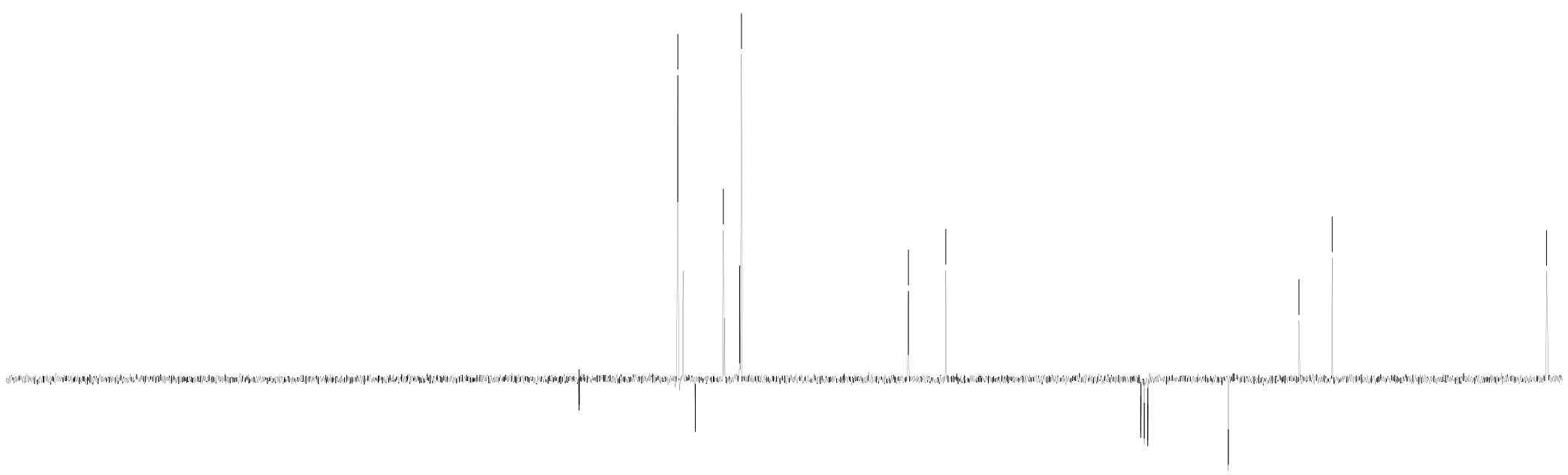

$210 \quad 200$ 
4-Ethoxy-1,1-dimethoxy-2-triethylsilyloxybut-2-ene 12d (300 MHz, $\mathrm{CDCl}_{3}$ )

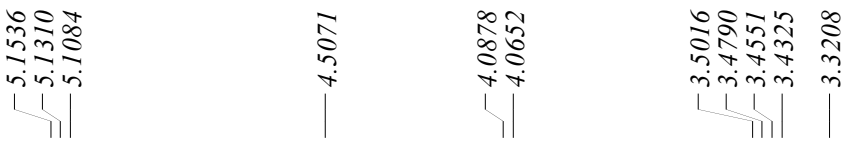
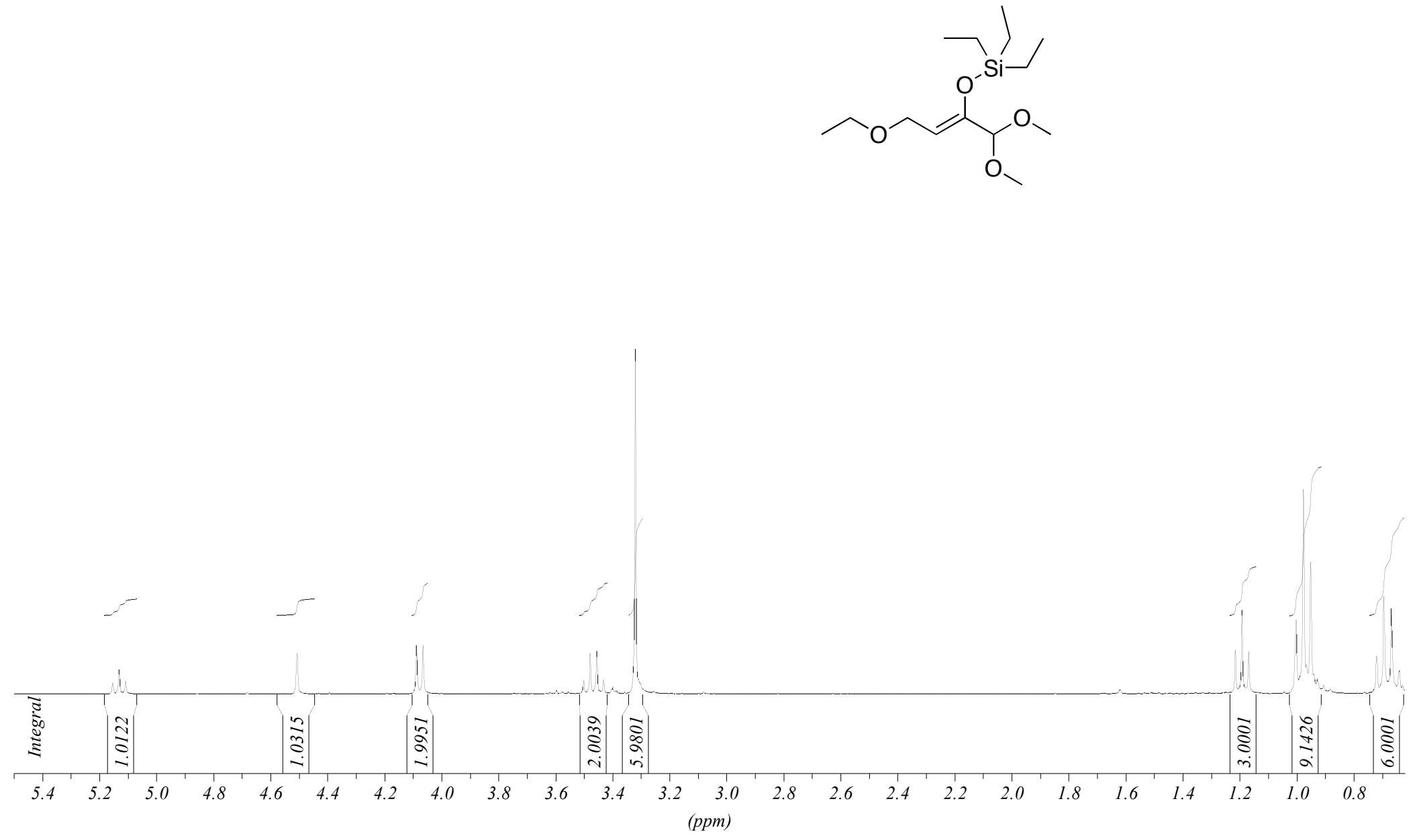
4-Ethoxy-1,1-dimethoxy-2-triethylsilyloxybut-2-ene 12d

$\left(75 \mathrm{MHz}, \mathrm{CDCl}_{3}\right)$

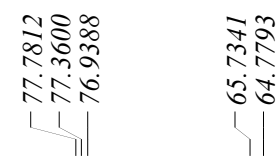

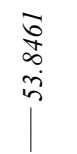

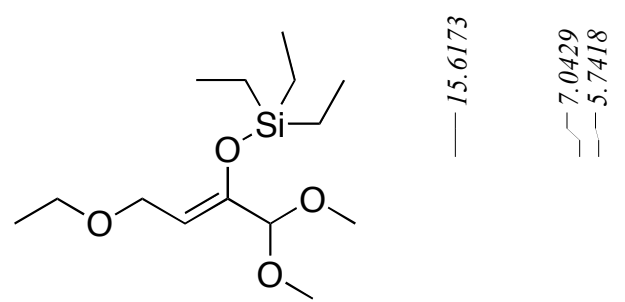

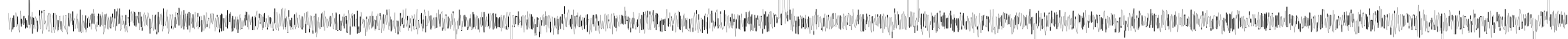


2-(tert-Butyldimethylsilyloxy)-4-ethoxy-1,1-dimethoxybut-2-ene 12e (300 MHz, $\mathrm{CDCl}_{3}$ )
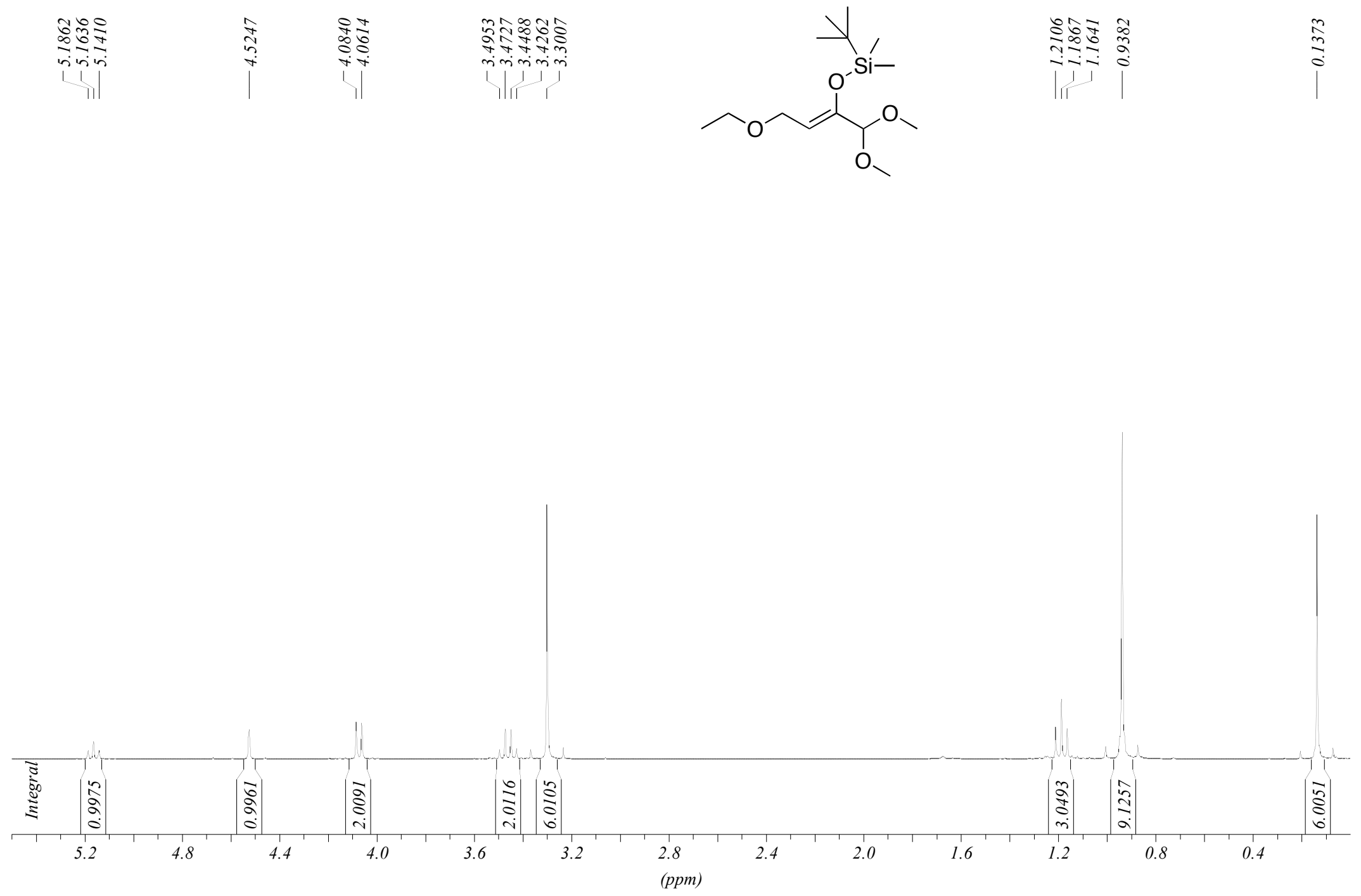
2-(tert-Butyldimethylsilyloxy)-4-ethoxy-1,1-dimethoxybut-2-ene 12e $\left(75 \mathrm{MHz}, \mathrm{CDCl}_{3}\right)$

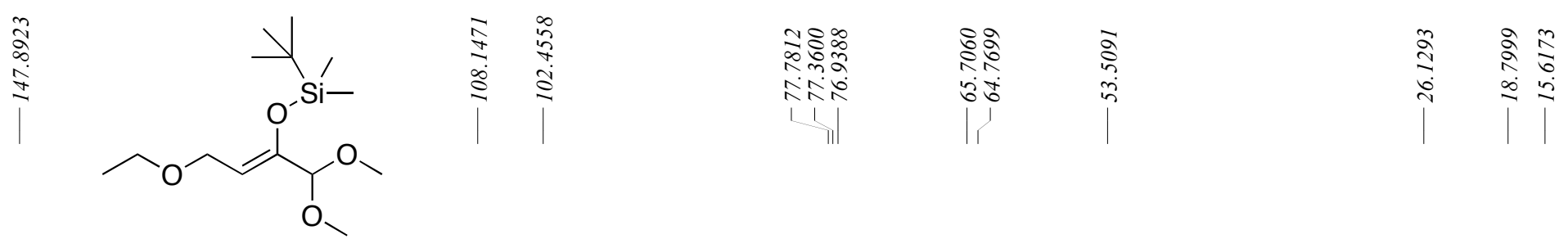

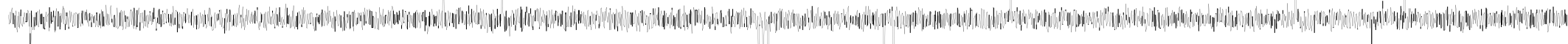

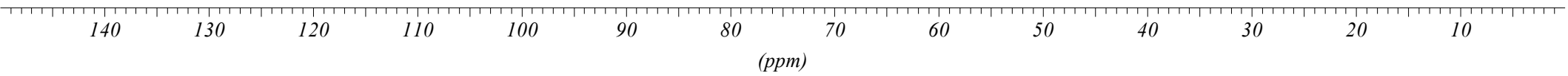


4-Ethoxy-1,1-dimethoxy-2-triisopropylsilyloxybut-2-ene $\mathbf{1 2 f}$ (300 MHz, $\mathrm{CDCl}_{3}$ )

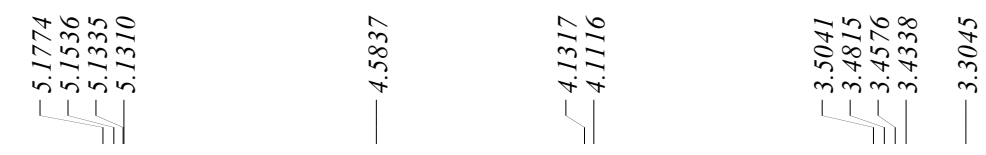

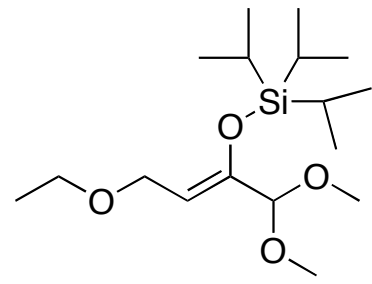

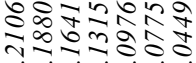

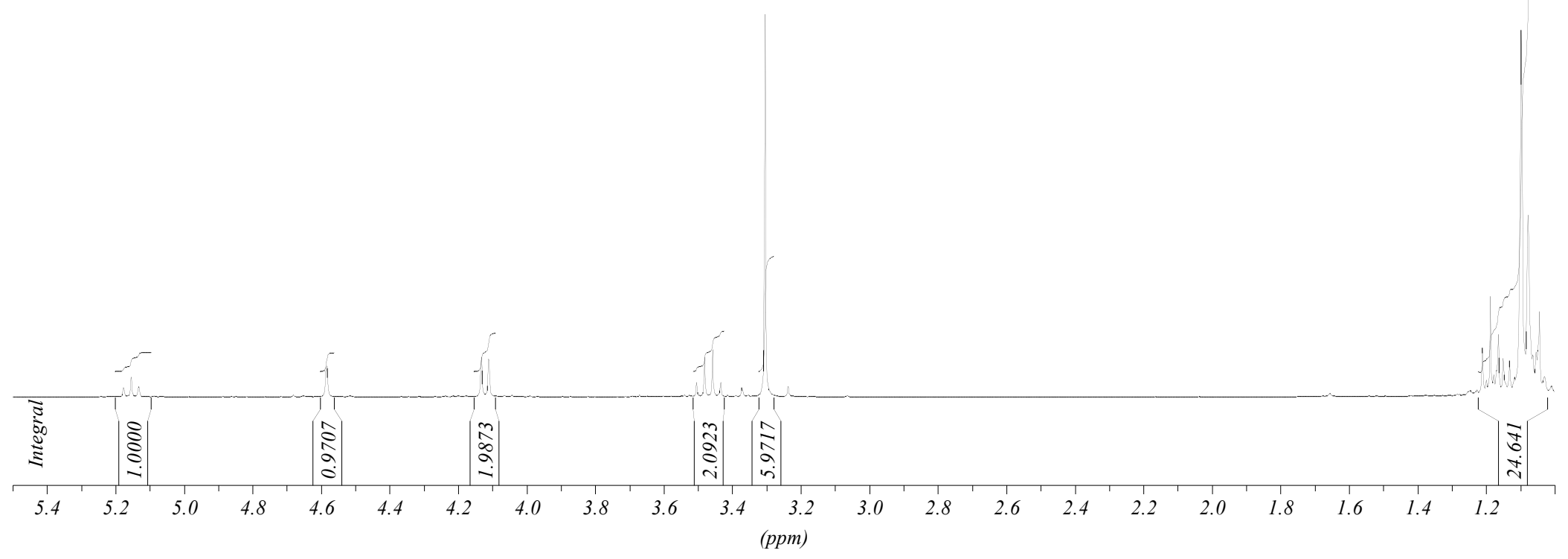


4-Ethoxy-1,1-dimethoxy-2-triisopropylsilyloxybut-2-ene $\mathbf{1 2 f}$

(75 MHz, $\mathrm{CDCl}_{3}$ )
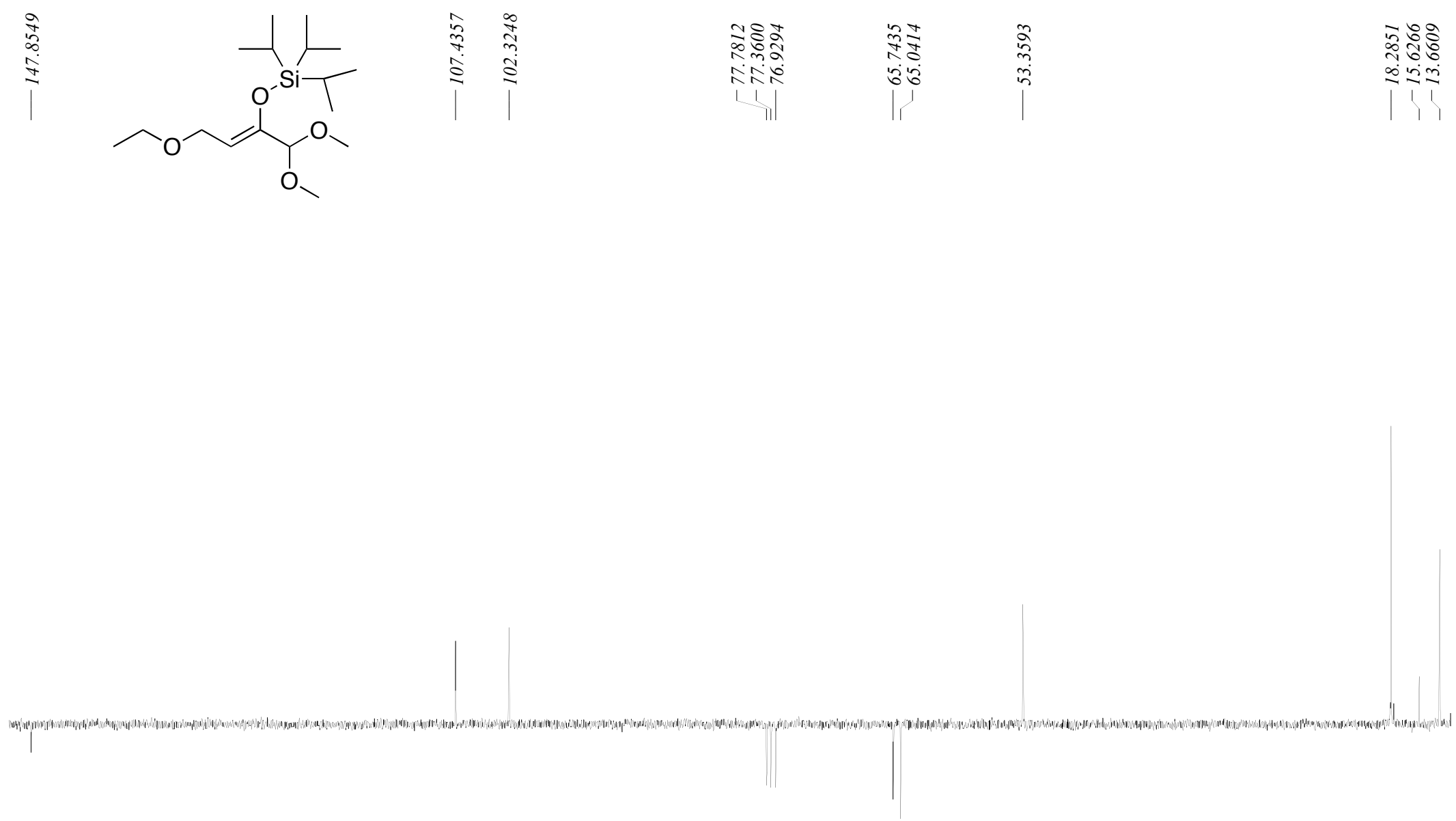

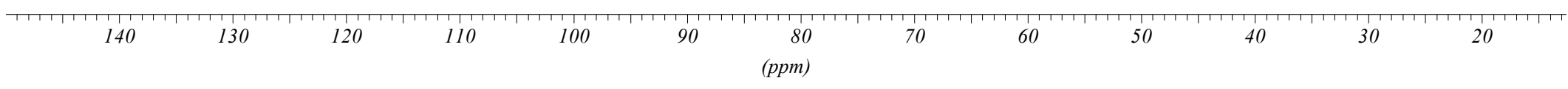


2-(tert-Butyldiphenylsilyloxy)-4-ethoxy-1,1-dimethoxybut-2-ene 12g (300 MHz, $\mathrm{CDCl}_{3}$ )
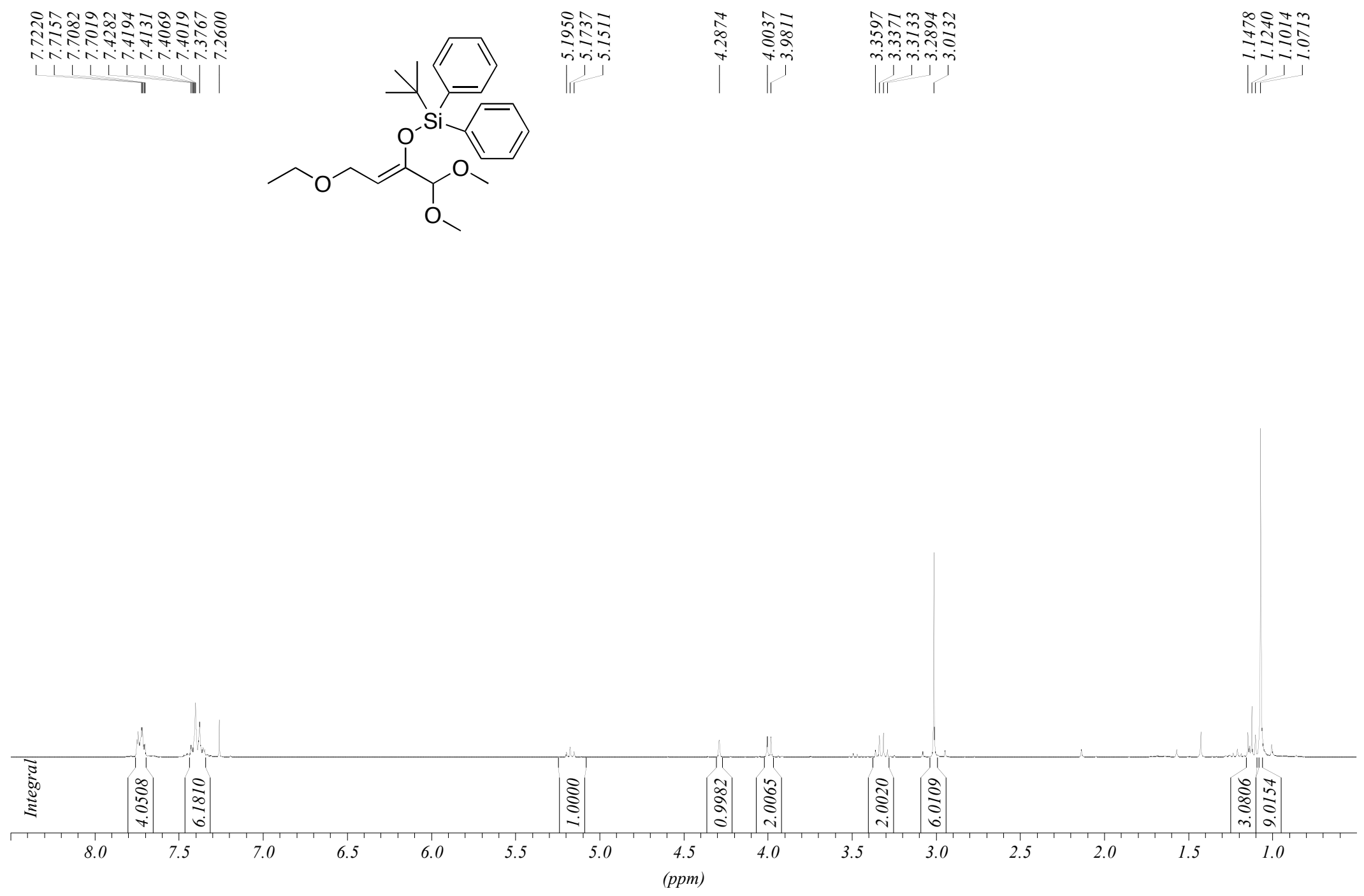
2-(tert-Butyldiphenylsilyloxy)-4-ethoxy-1,1-dimethoxybut-2-ene 12g (75 MHz, $\mathrm{CDCl}_{3}$ )
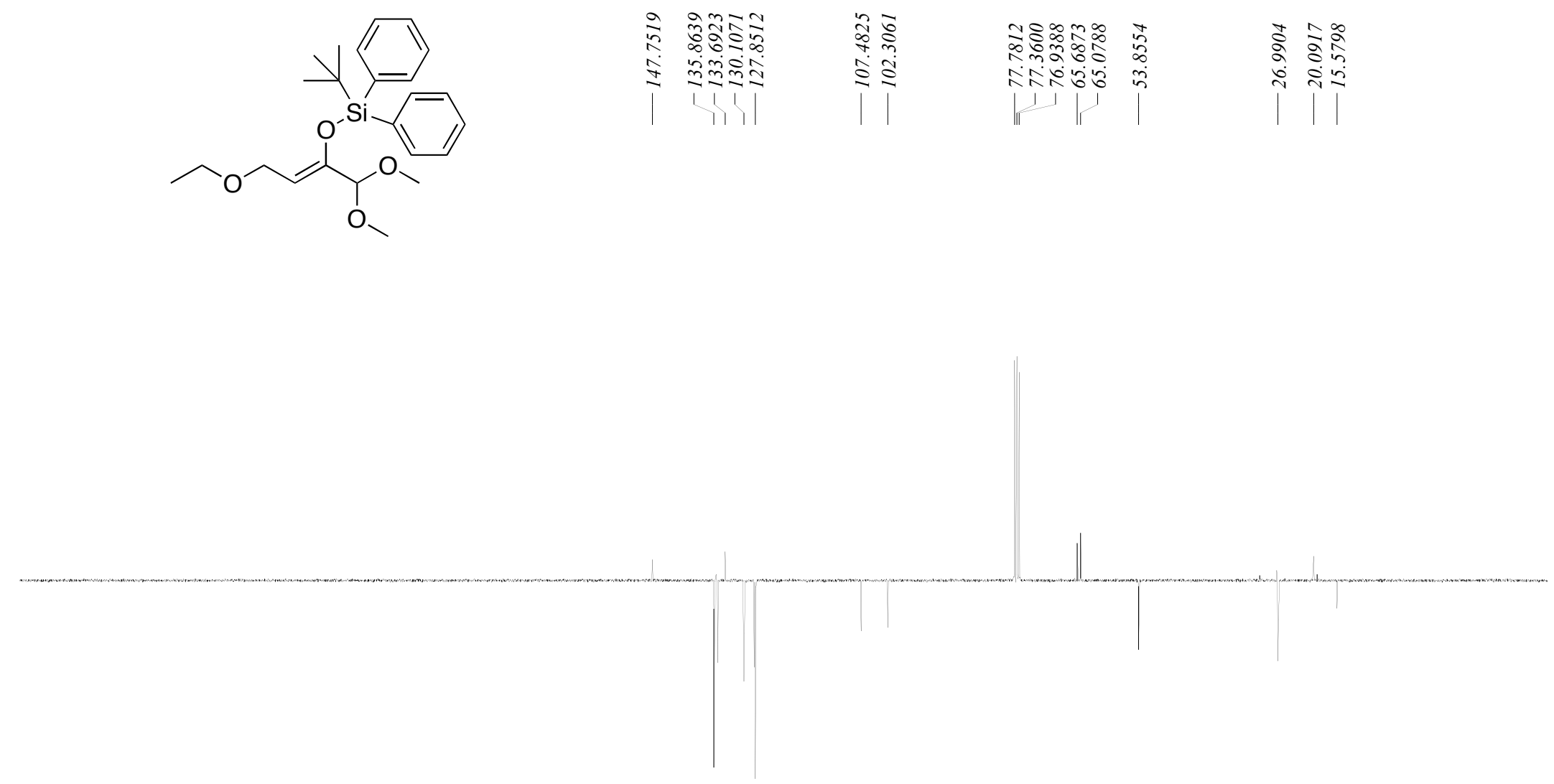

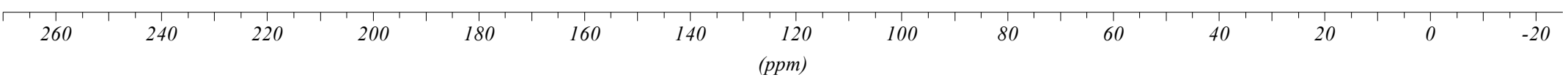


4-Ethoxy-1-[bis-(1-ethylpropyloxy)]-2-triethylsilyloxybut-2-ene $\mathbf{1 2 h}$ $\left(200 \mathrm{MHz}, \mathrm{CDCl}_{3}\right)$
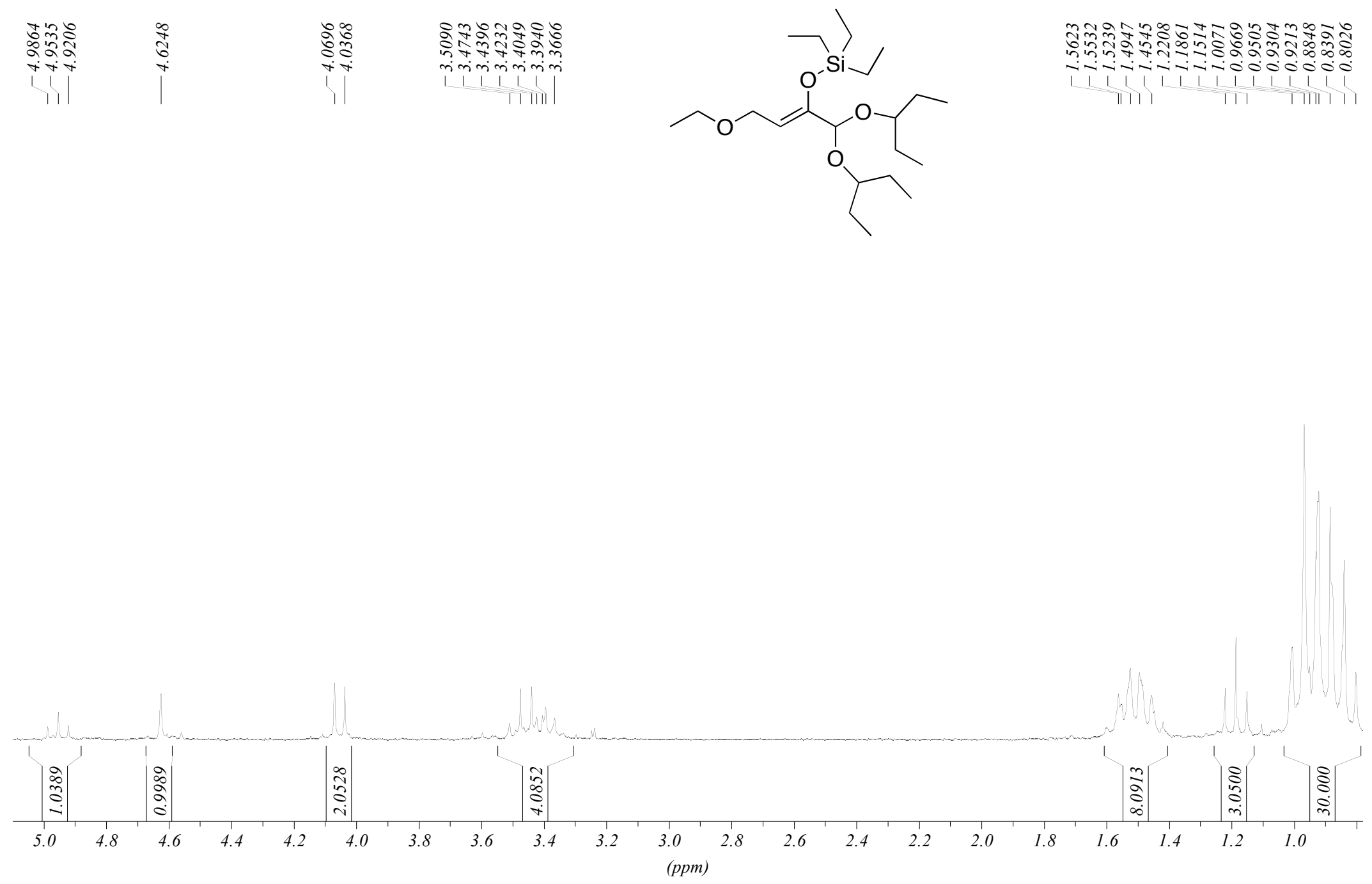
4-Ethoxy-1-[bis-(1-ethylpropyloxy)]-2-triethylsilyloxybut-2-ene $\mathbf{1 2 h}$

$\left(50 \mathrm{MHz}, \mathrm{CDCl}_{3}\right)$

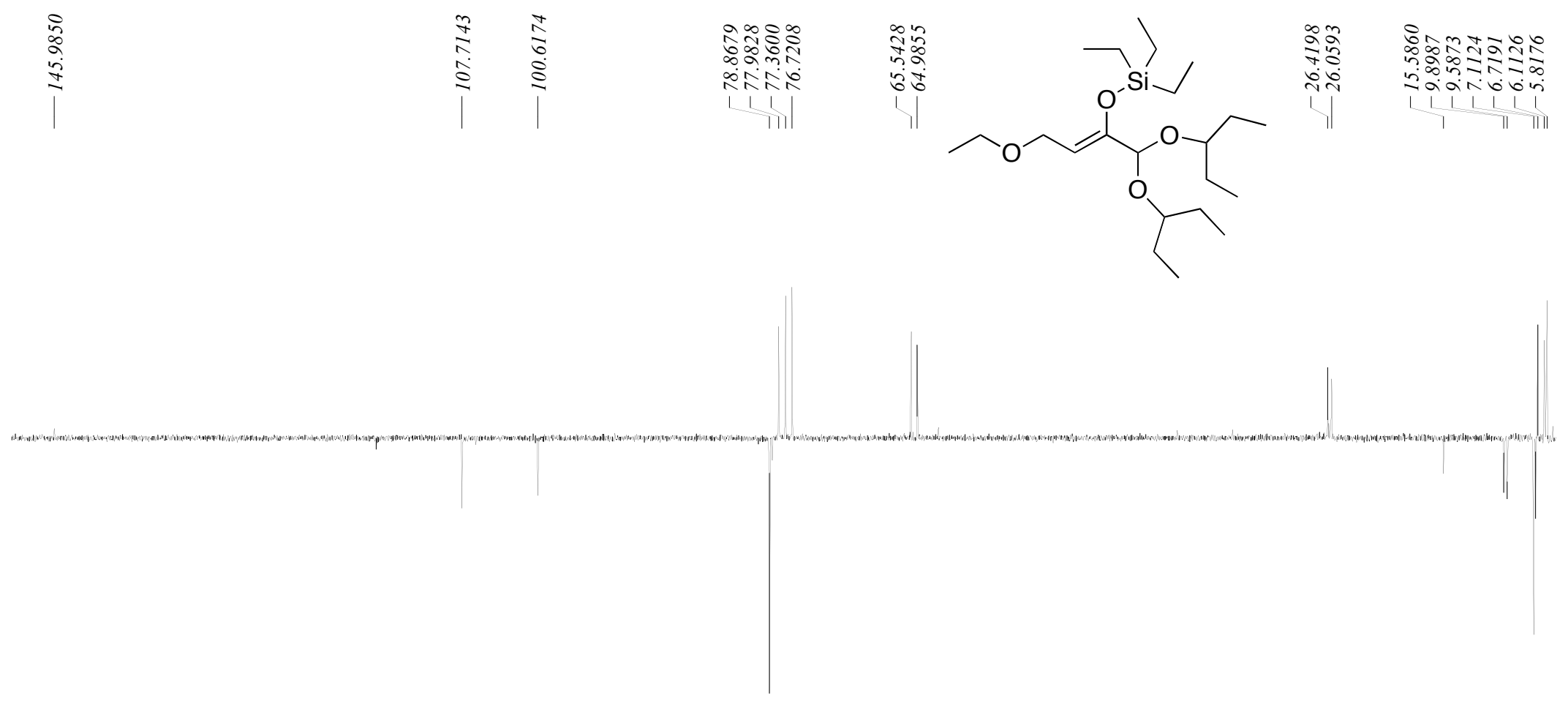

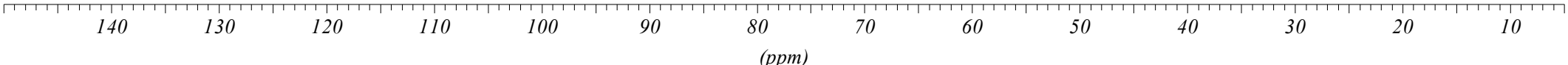


1,4-Dimethoxy-2-triethylsilyloxy-1,3-butadiene 13a

(300 MHz, $\mathrm{CDCl}_{3}$ )
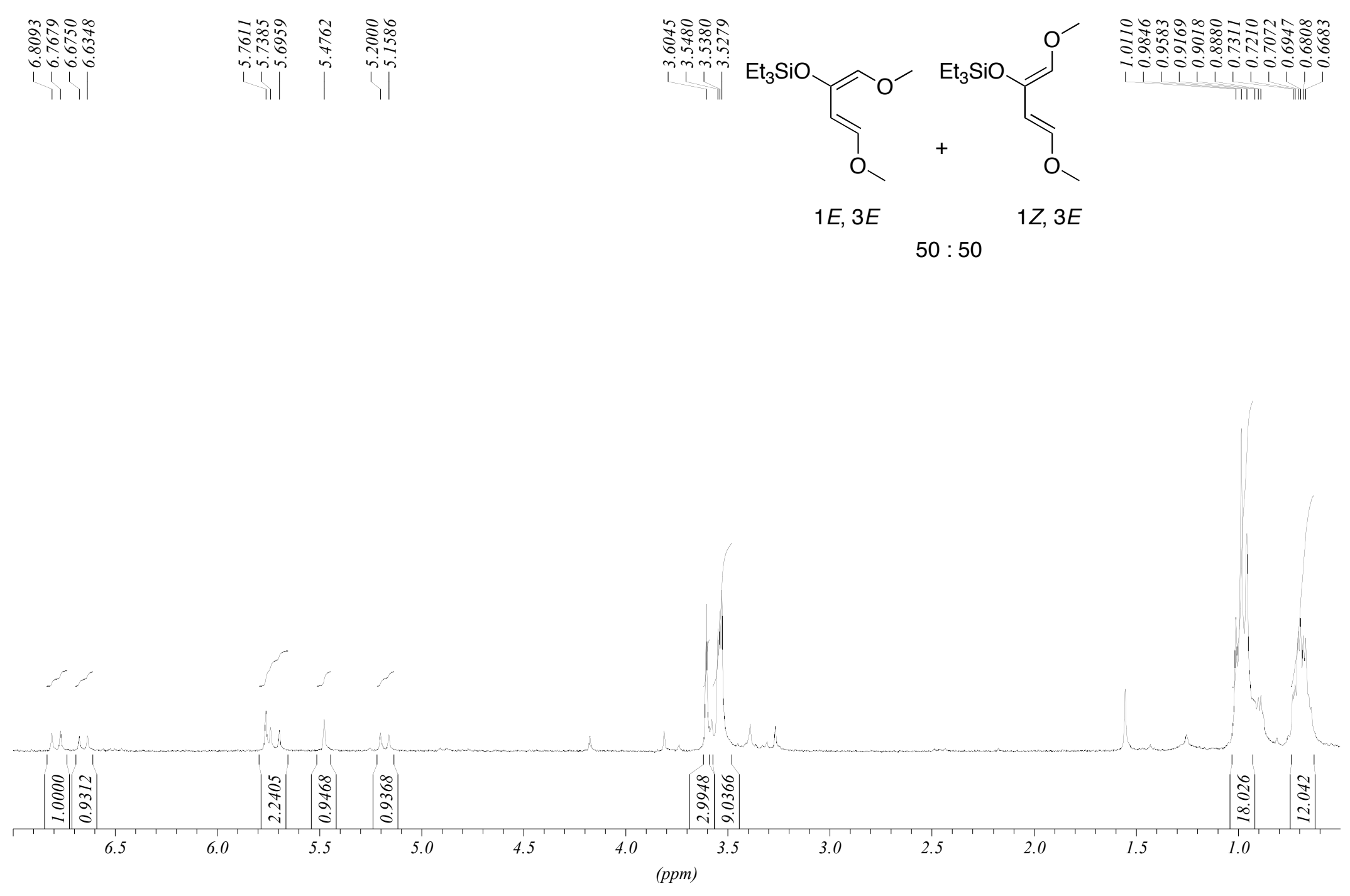
S35

1,4-Dimethoxy-2-triethylsilyloxy-1,3-butadiene 13a

(75 MHz, $\mathrm{CDCl}_{3}$ )
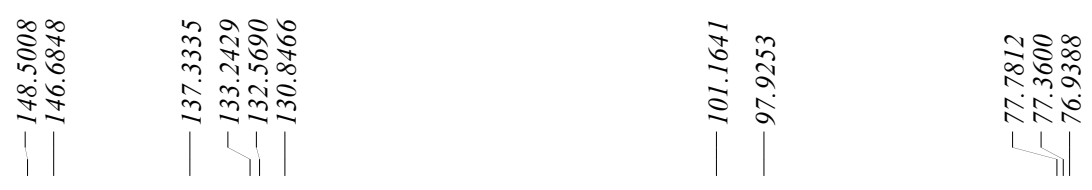

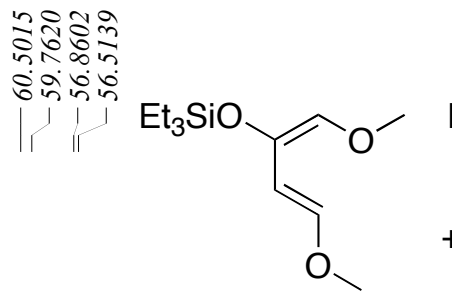

$1 E, 3 E$

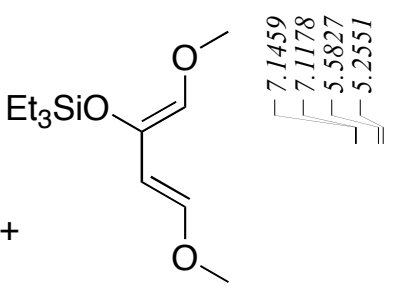

$1 Z, 3 E$

$50: 50$

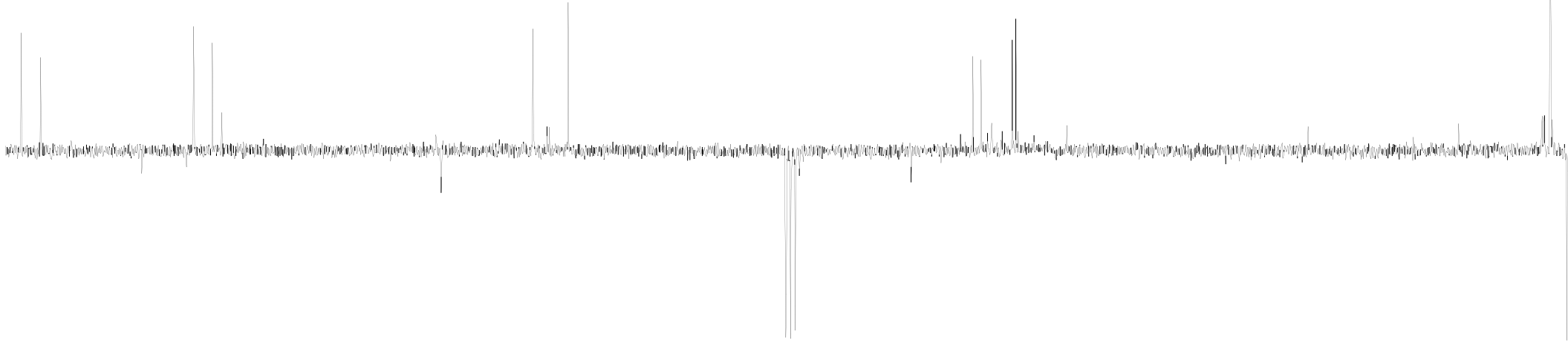

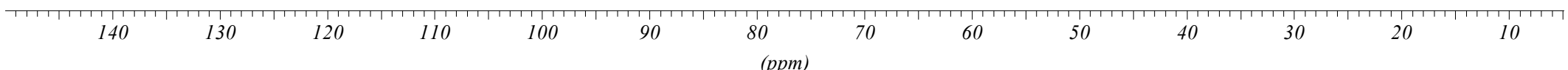


1,4-Dimethoxy-2-triisobutylsilyloxy-1,3-butadiene 13b

(200 MHz, $\mathrm{CDCl}_{3}$ )

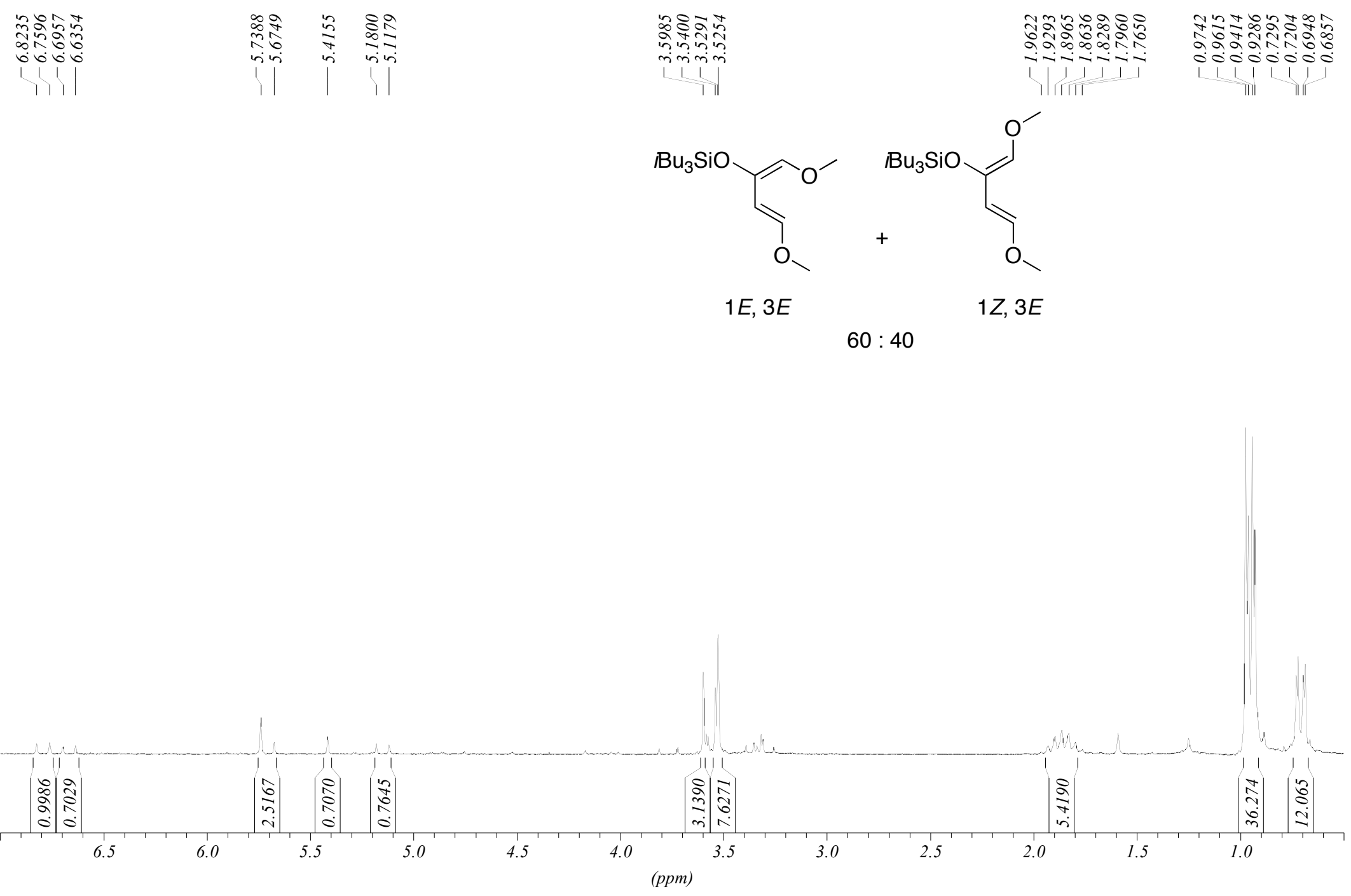


1,4-Dimethoxy-2-triisobutylsilyloxy-1,3-butadiene $\mathbf{1 3 b}$

(50 $\left.\mathrm{MHz}, \mathrm{CDCl}_{3}\right)$
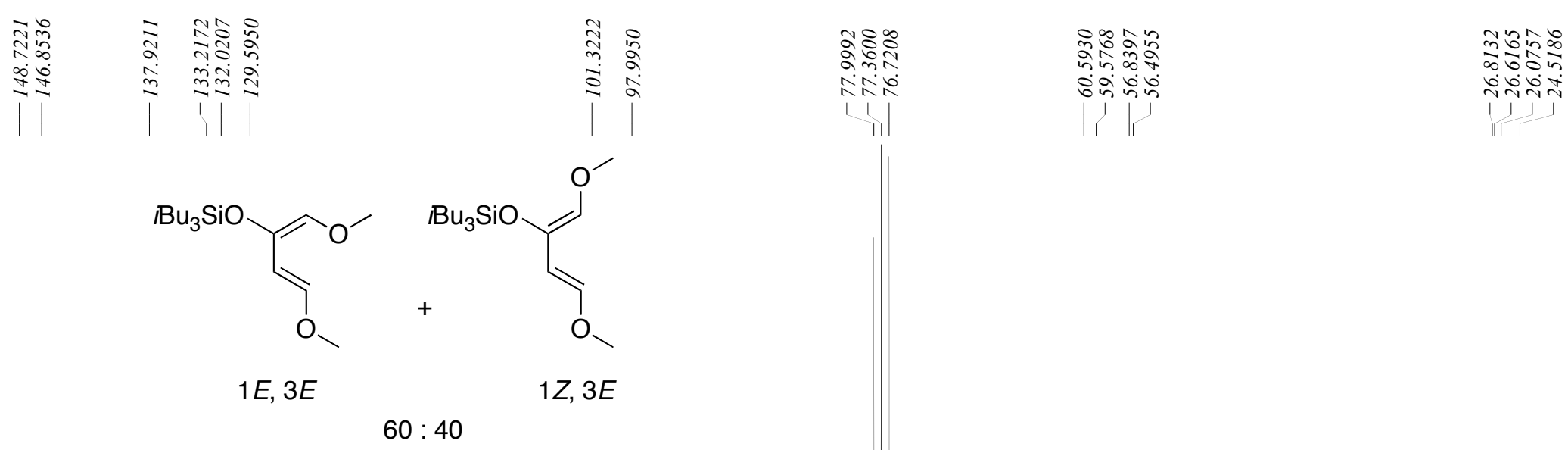

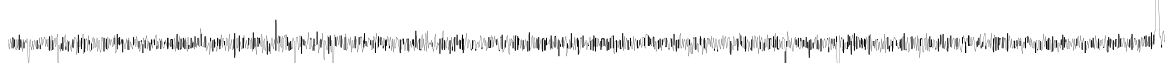
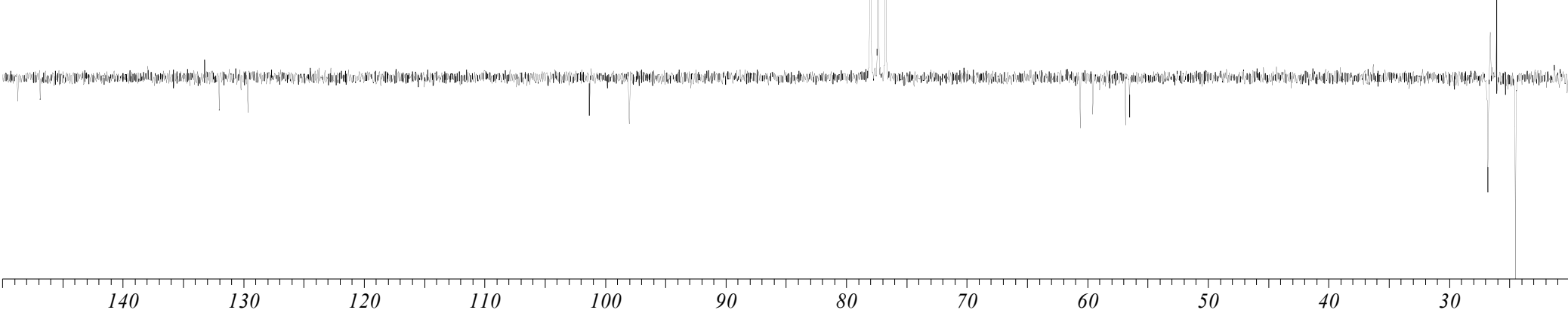
4-Ethoxy-1-methoxy-2-triethylsilyloxy-1,3-butadiene 13d

$\left(200 \mathrm{MHz}, \mathrm{CDCl}_{3}\right)$
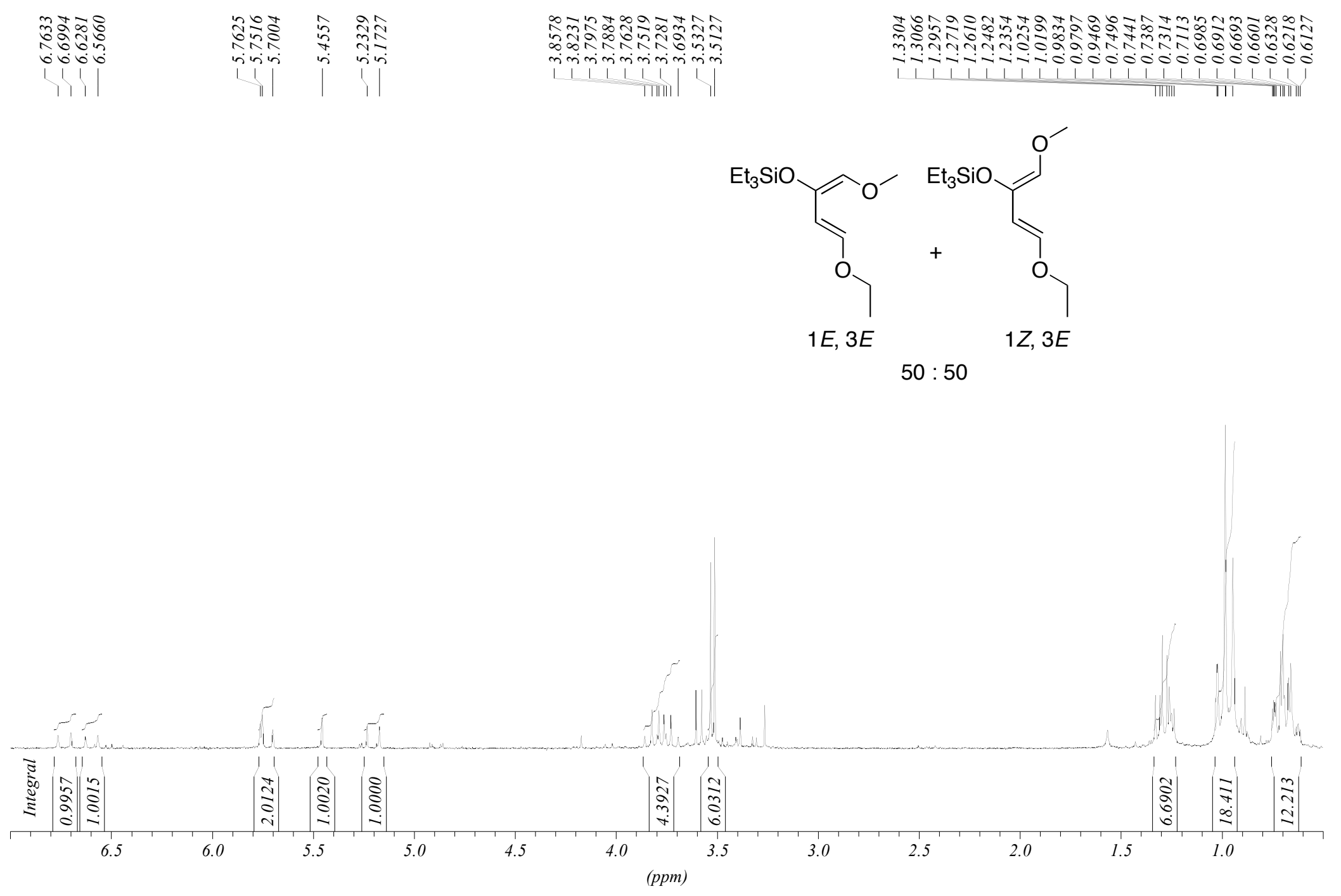
4-Ethoxy-1-methoxy-2-triethylsilyloxy-1,3-butadiene 13d

(75 MHz, $\mathrm{CDCl}_{3}$ )
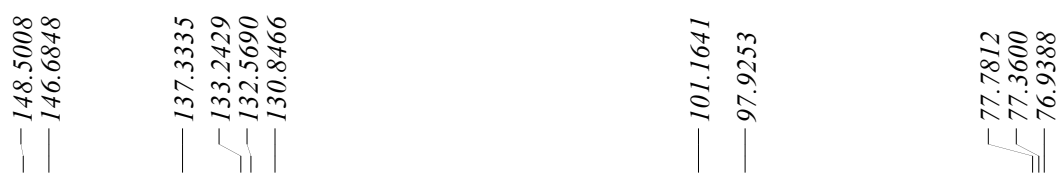

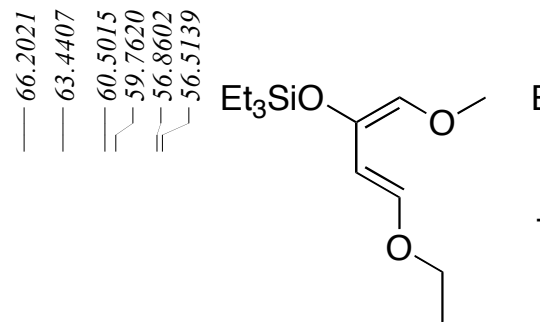

$1 E, 3 E$

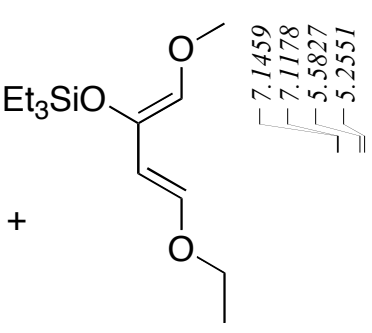

$1 Z, 3 E$

$50: 50$

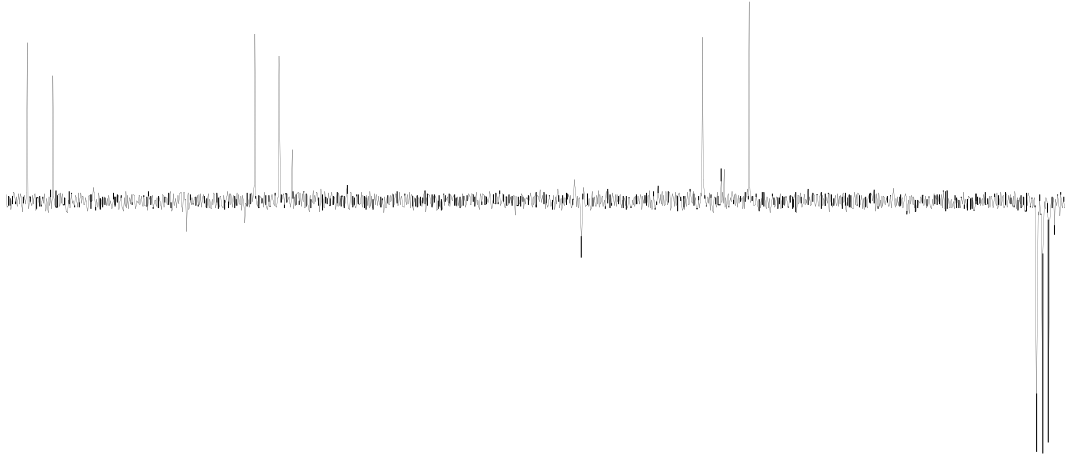


2-tertButyldimethylsilyloxy-4-ethoxy-1-methoxy-1,3-butadiene 13e (300 MHz, $\mathrm{CDCl}_{3}$ )

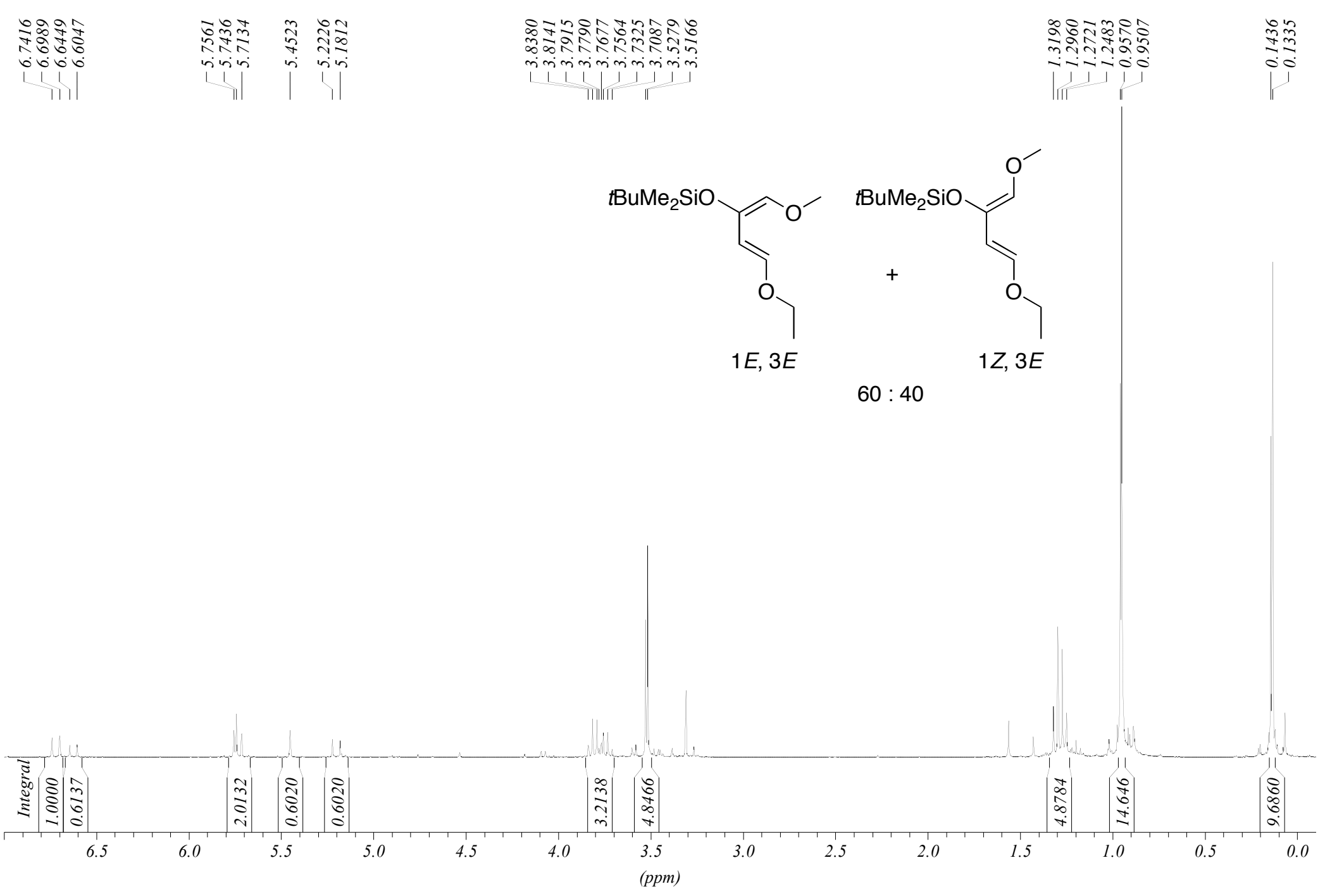


2-tertButyldimethylsilyloxy-4-ethoxy-1-methoxy-1,3-butadiene 13e (75 MHz, $\mathrm{CDCl}_{3}$ )
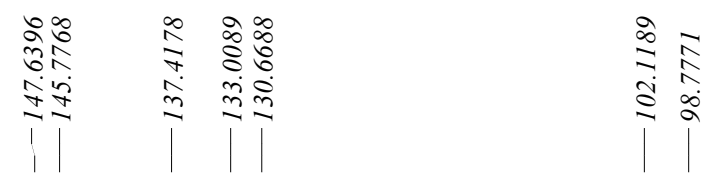

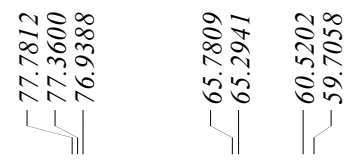
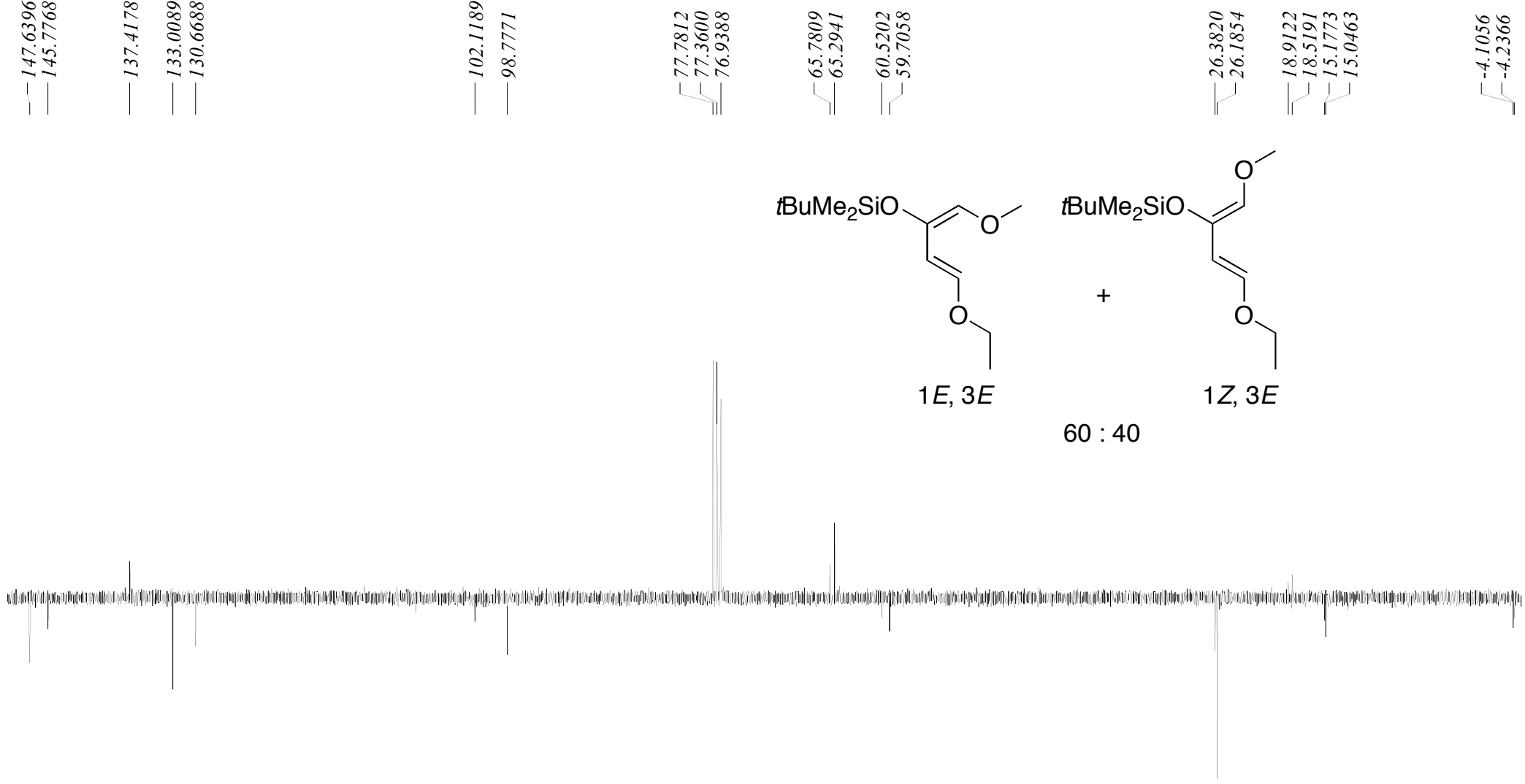

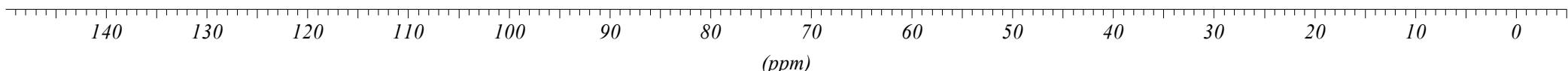


4-Ethoxy-1-methoxy-2-triisopropylsilyloxy-1,3-butadiene $\mathbf{1 3 f}$ $\left(200 \mathrm{MHz}, \mathrm{CDCl}_{3}\right)$

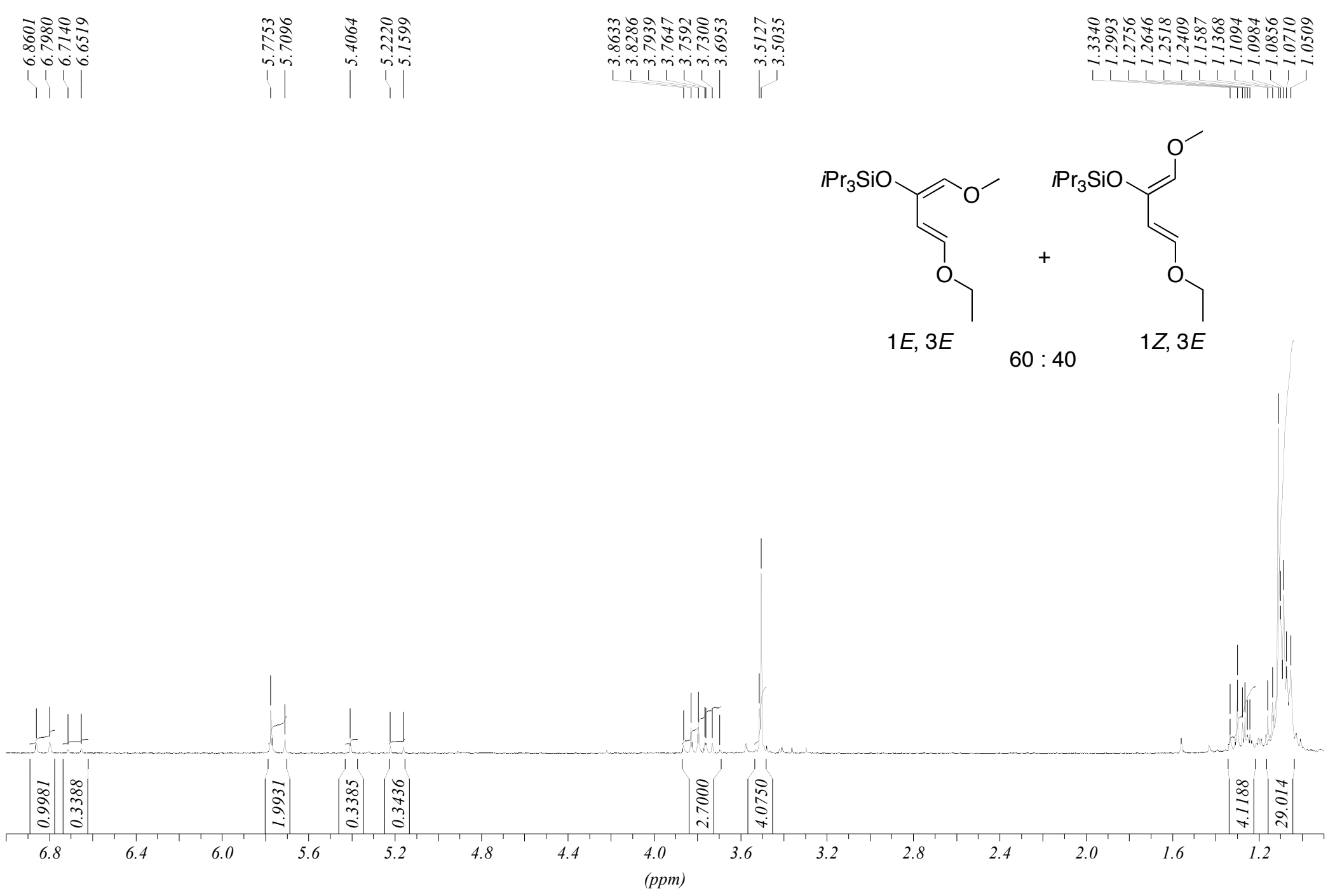


4-Ethoxy-1-methoxy-2-triisopropylsilyloxy-1,3-butadiene $\mathbf{1 3 f}$

(50 $\left.\mathrm{MHz}, \mathrm{CDCl}_{3}\right)$

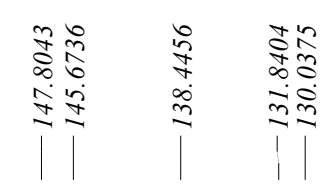
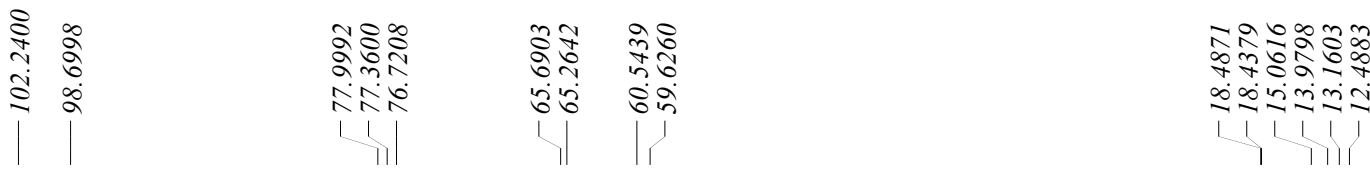

t.

-
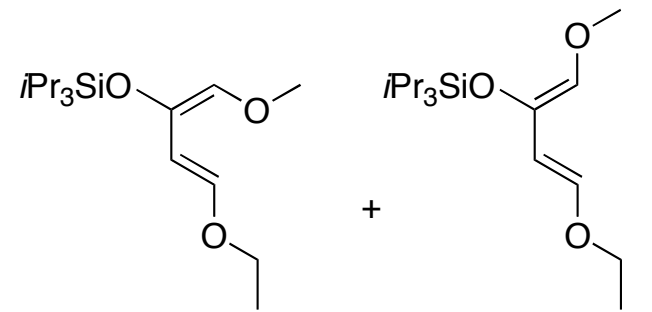

$1 E, 3 E$

$60: 40$

$1 Z, 3 E$

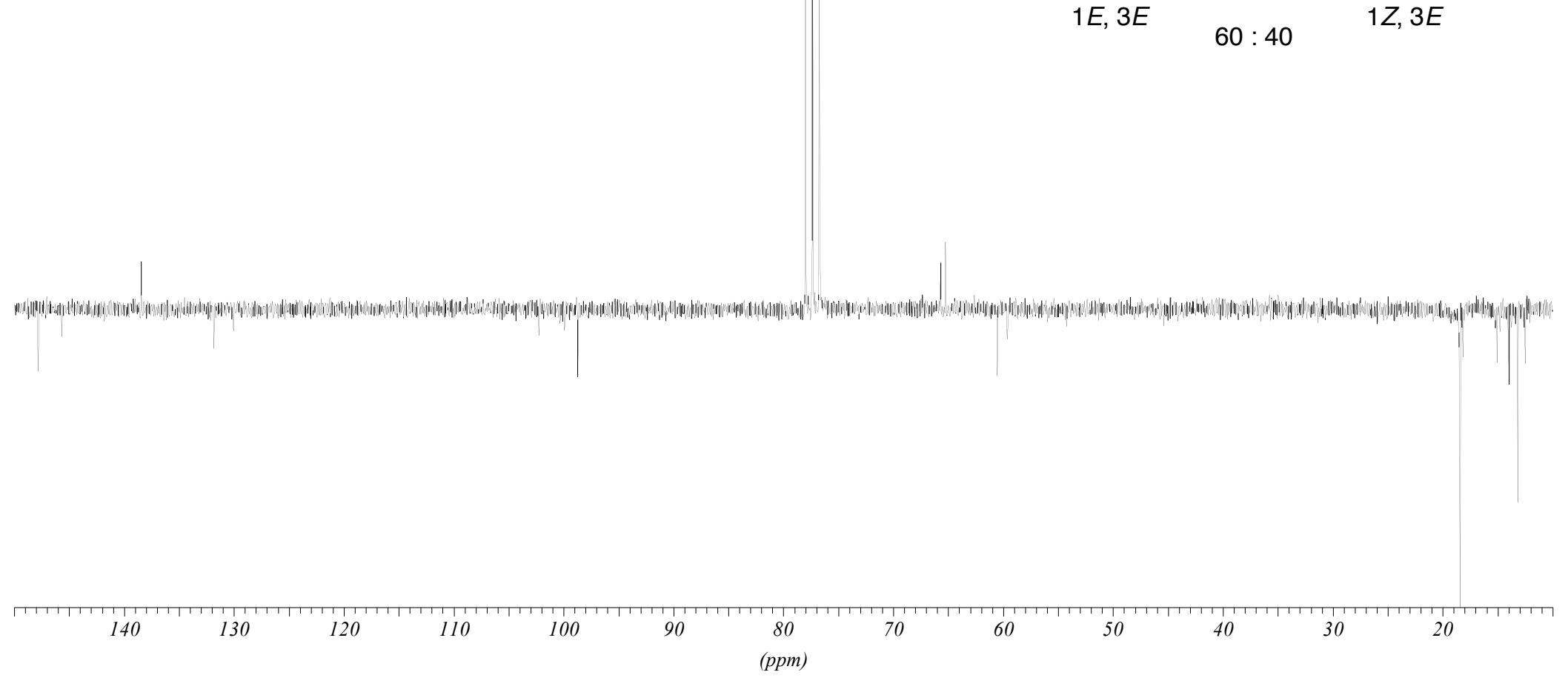


2-tertButyldiphenylsilyloxy-4-ethoxy-1-methoxy-1,3-butadiene 13g (300 MHz, $\mathrm{CDCl}_{3}$ )

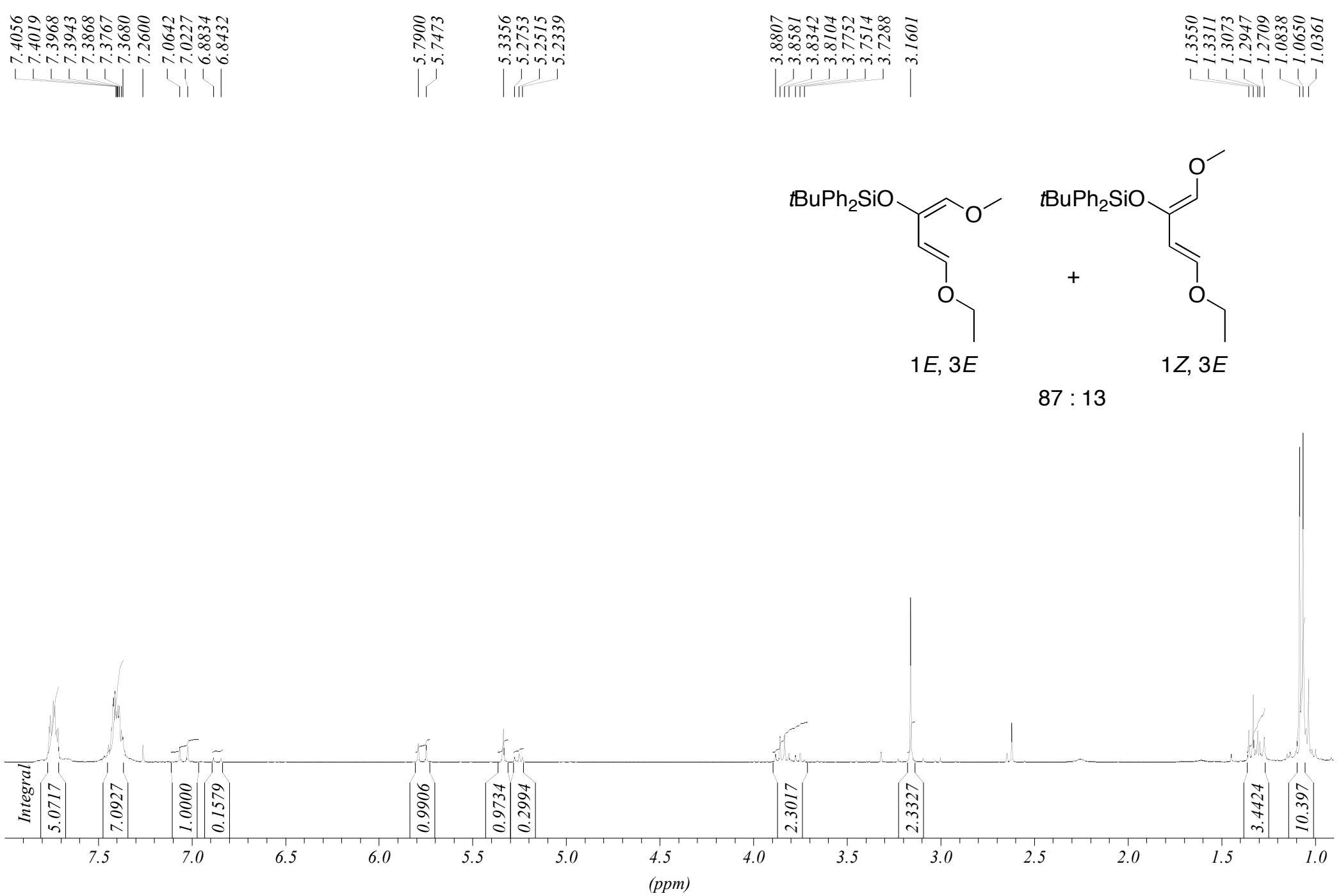


2-tertButyldiphenylsilyloxy-4-ethoxy-1-methoxy-1,3-butadiene 13g (75 MHz, $\mathrm{CDCl}_{3}$ )
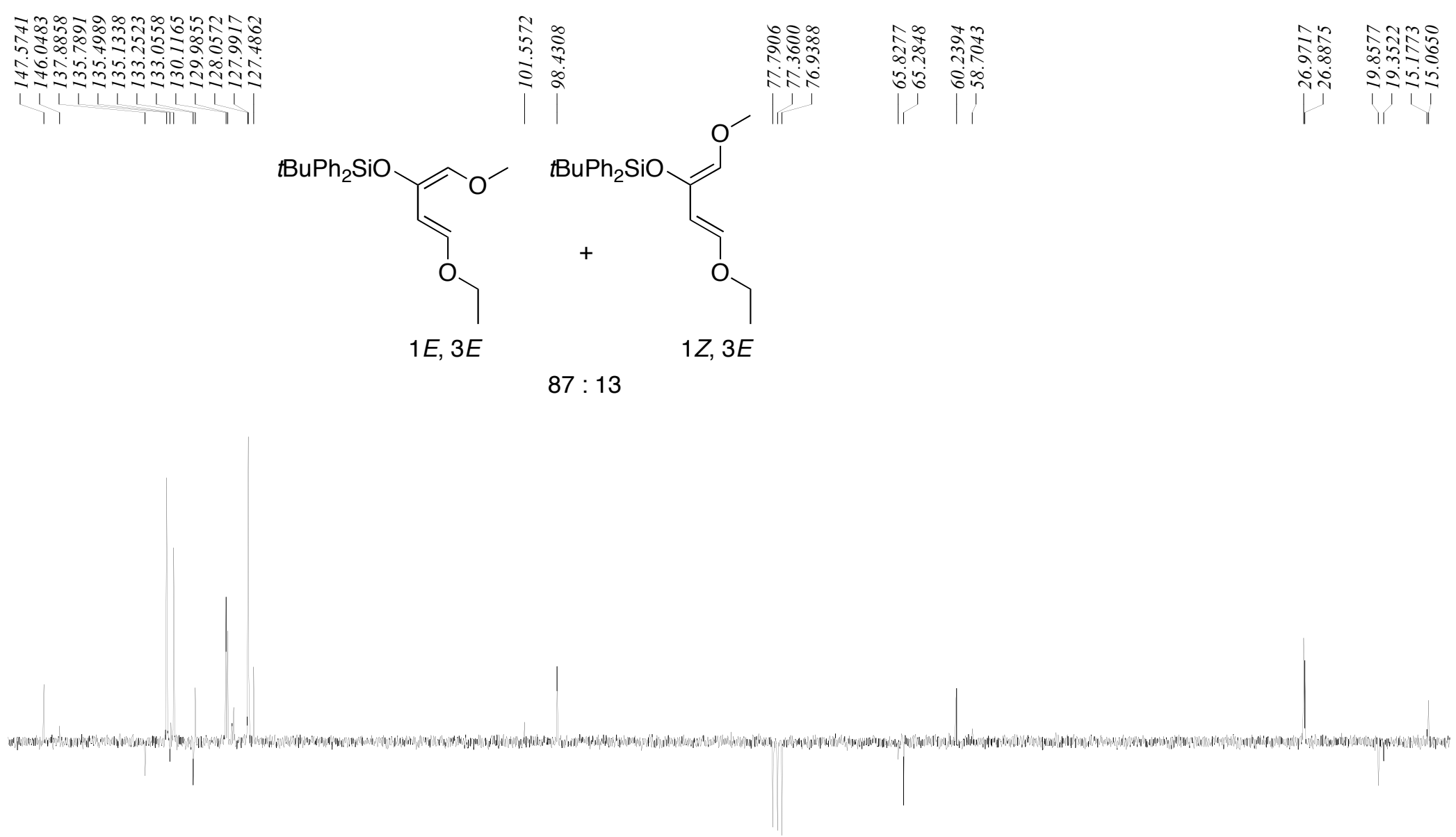

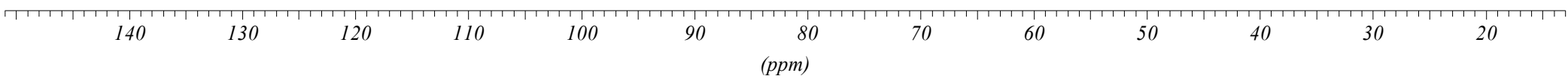


4-Ethoxy-1-(1-ethylpropyloxy)-2-triethylsilyloxy-1,3-butadiene $\mathbf{1 3 h}$

$\left(300 \mathrm{MHz}, \mathrm{CDCl}_{3}\right.$ )

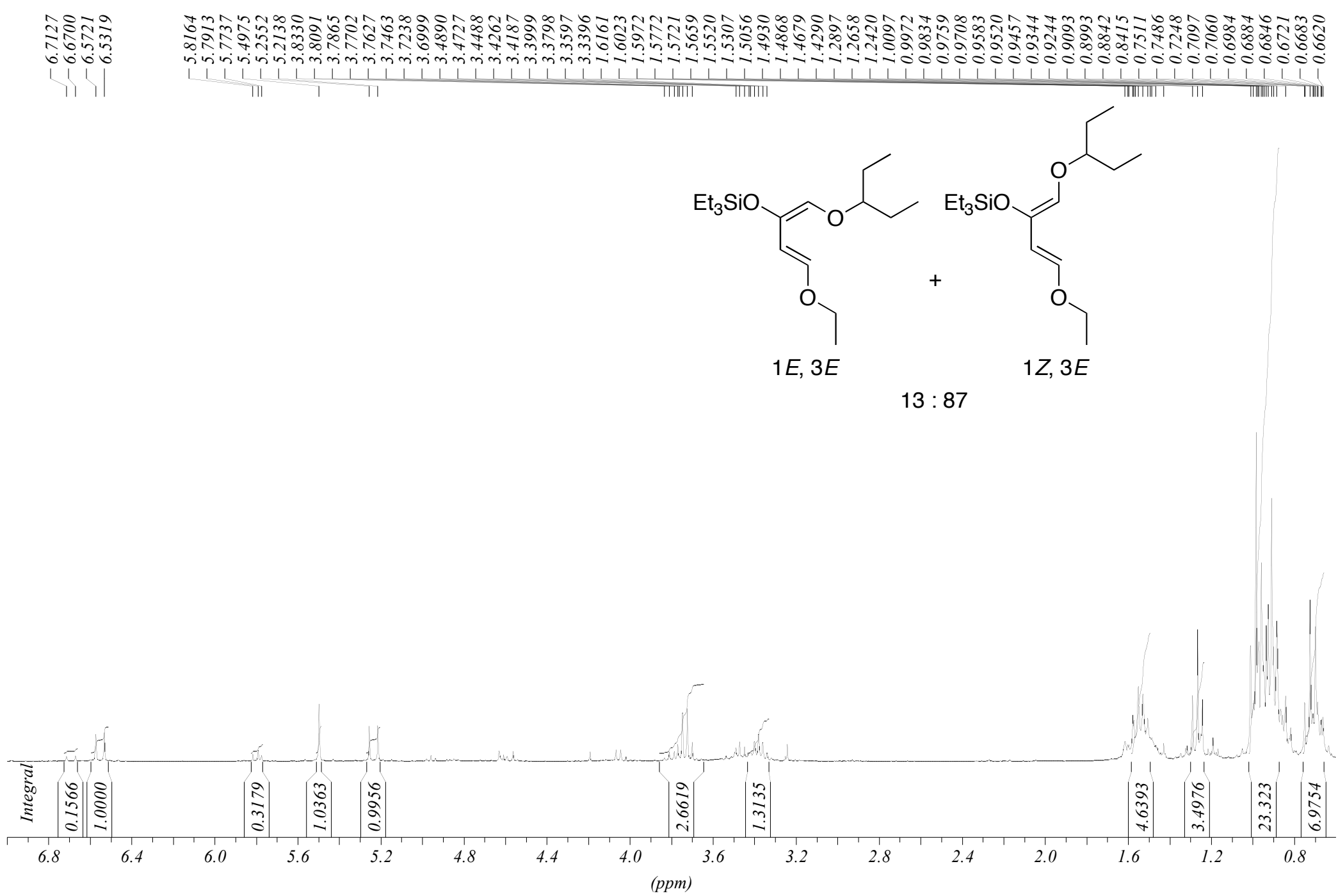


4-Ethoxy-1-(1-ethylpropyloxy)-2-triethylsilyloxy-1,3-butadiene 13h (75 MHz, $\mathrm{CDCl}_{3}$ )

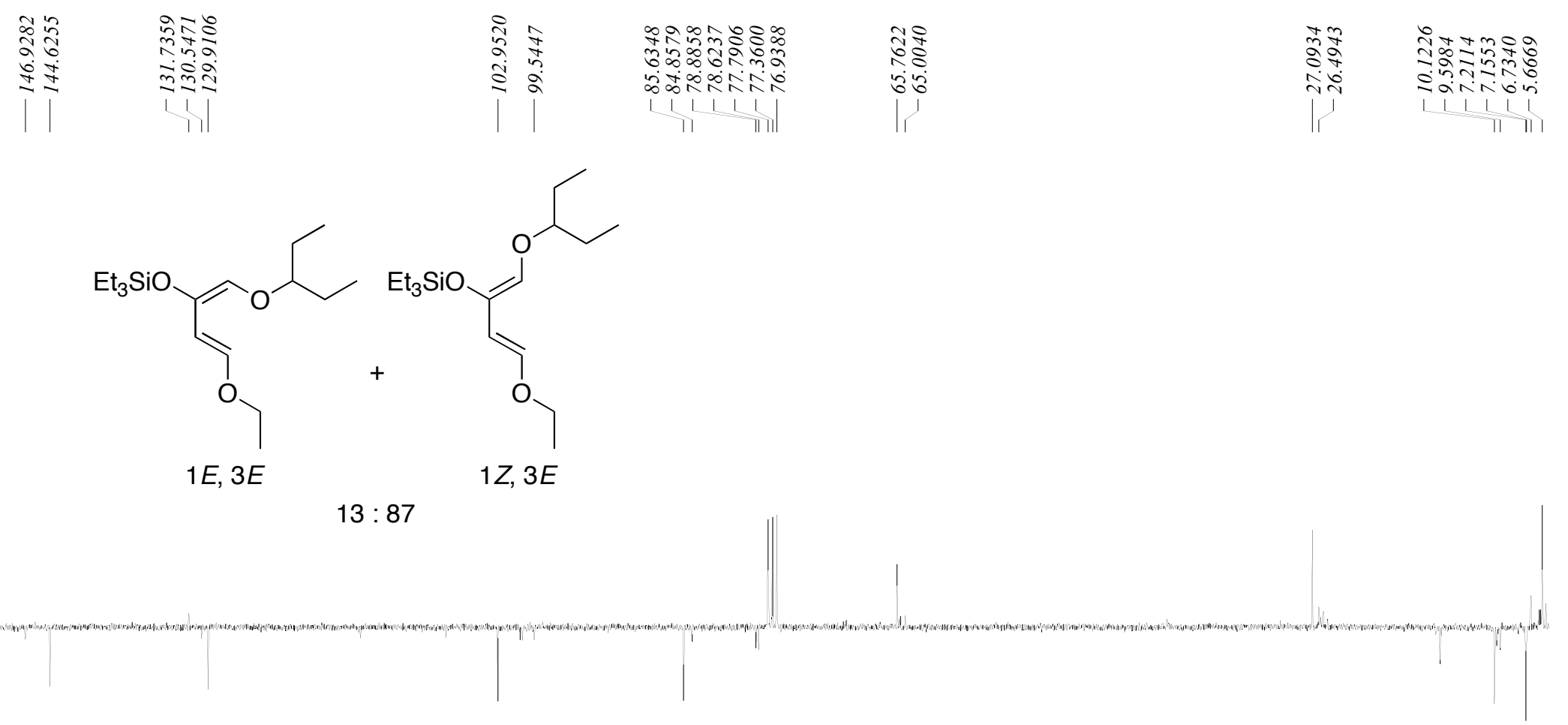

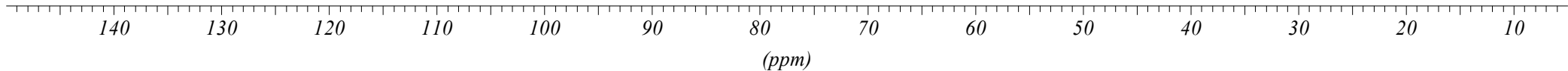


7-Ethoxy-4-methoxy-2-methyl-5-triethylsilyloxy-3a,4,7,7a-tetrahydroisoindole-1,3-dione 28 trans (300 MHz, $\mathrm{CDCl}_{3}$ )
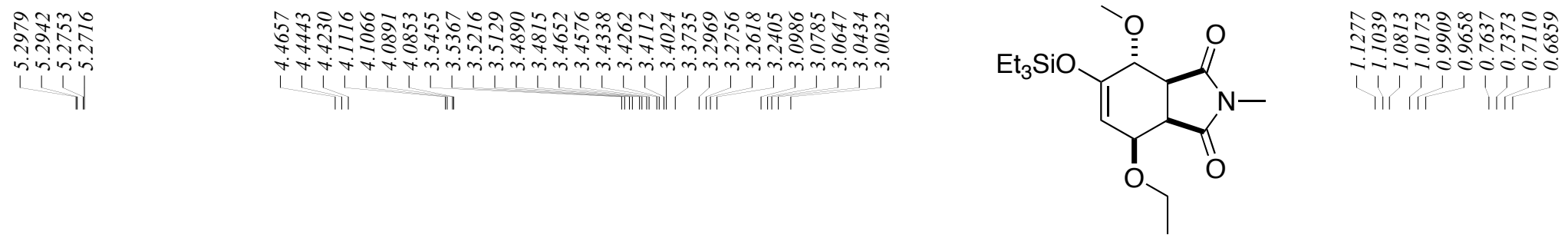

(1)

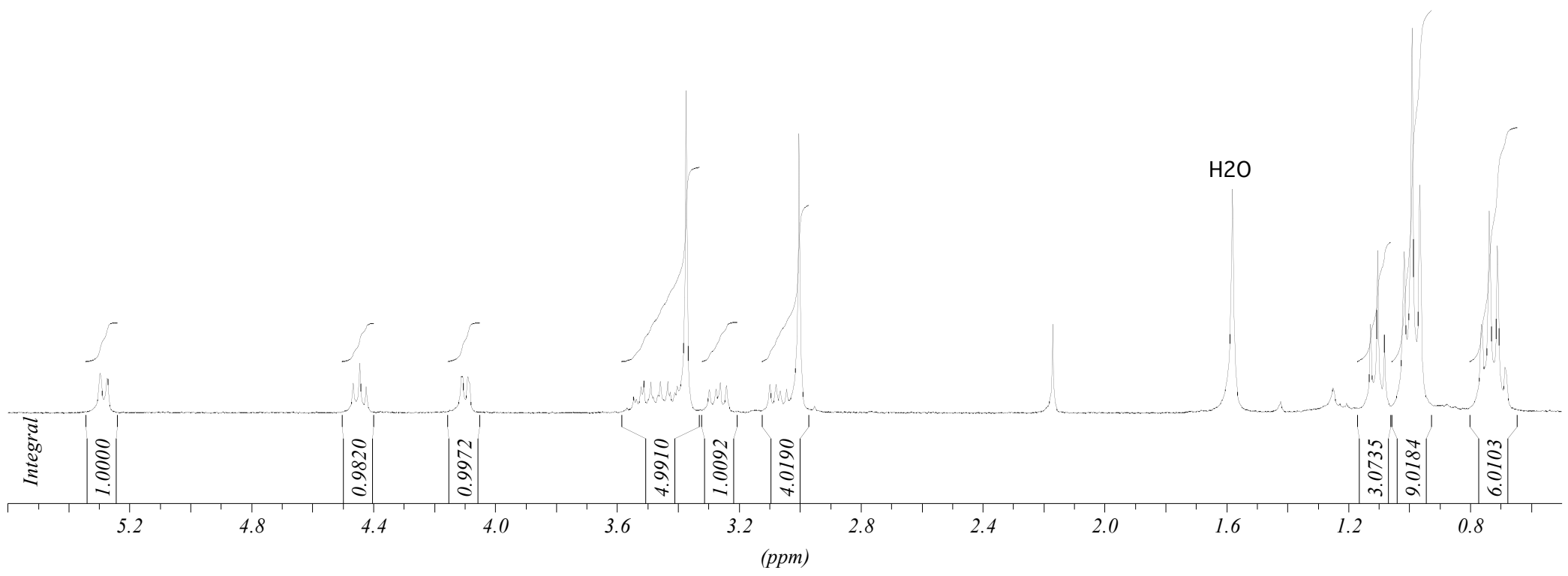


7-Ethoxy-4-methoxy-2-methyl-5-triethylsilyloxy-3a,4,7,7a-tetrahydroisoindole-1,3-dione 28 trans $\left(75 \mathrm{MHz}, \mathrm{CDCl}_{3}\right.$ )

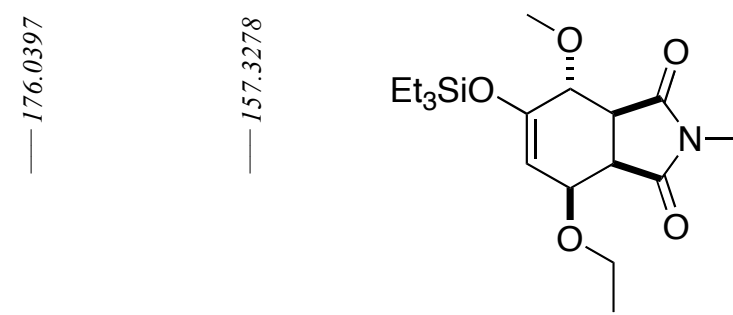

1
$\substack{2 \\ 0 \\ \vdots \\ 2}$

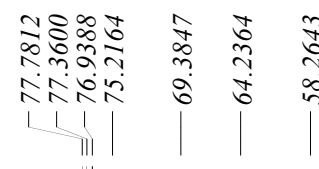

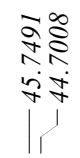

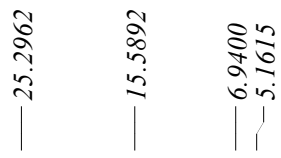

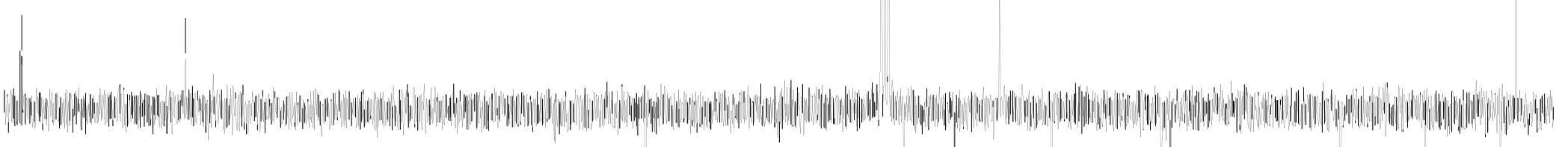

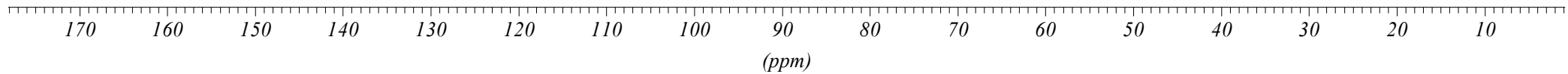


7-Ethoxy-4-methoxy-2-methyl-5-triethylsilyloxy-3a,4,7,7a-tetrahydroisoindole-1,3-dione 28 cis $\left(300 \mathrm{MHz}, \mathrm{CDCl}_{3}\right)$
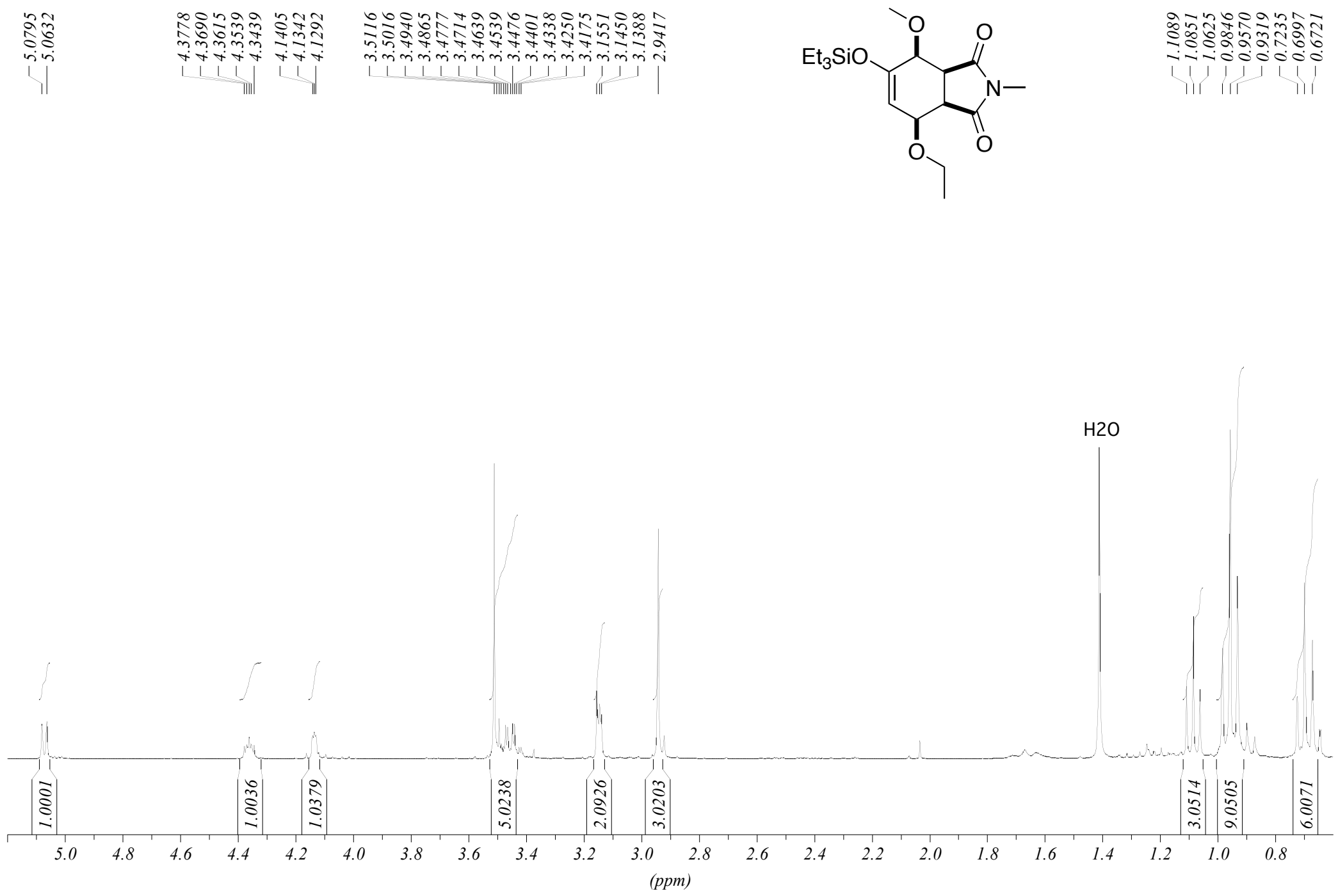
7-Ethoxy-4-methoxy-2-methyl-5-triethylsilyloxy-3a,4,7,7a-tetrahydroisoindole-1,3-dione 28 cis (75 MHz, $\mathrm{CDCl}_{3}$ )

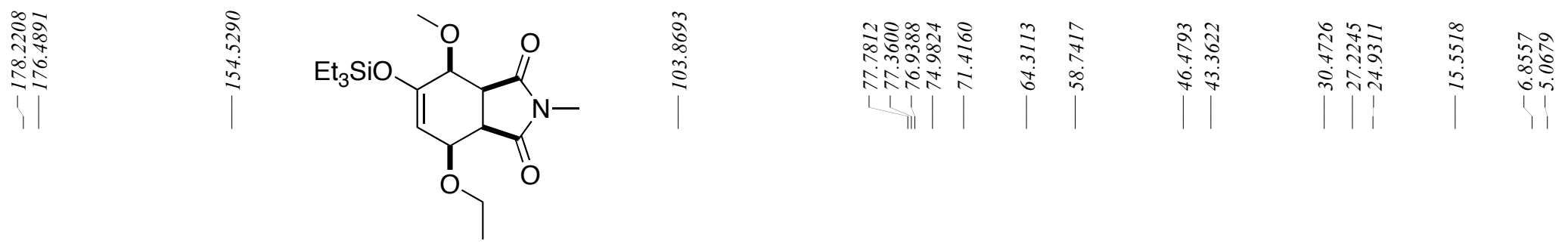

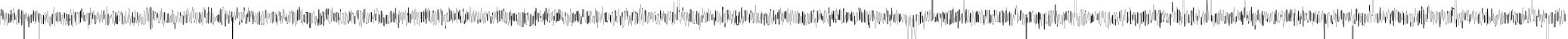
19

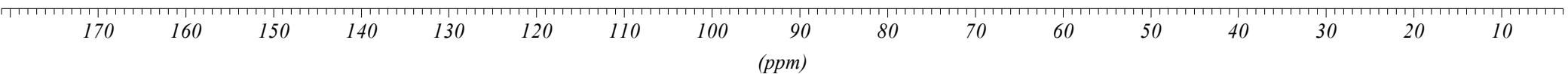


2-Ethoxy-5-methoxy-4-triethylsilyloxycyclohex-3-enecarboxylic acid phenyl ester 29 trans $\left(300 \mathrm{MHz}, \mathrm{CDCl}_{3}\right)$
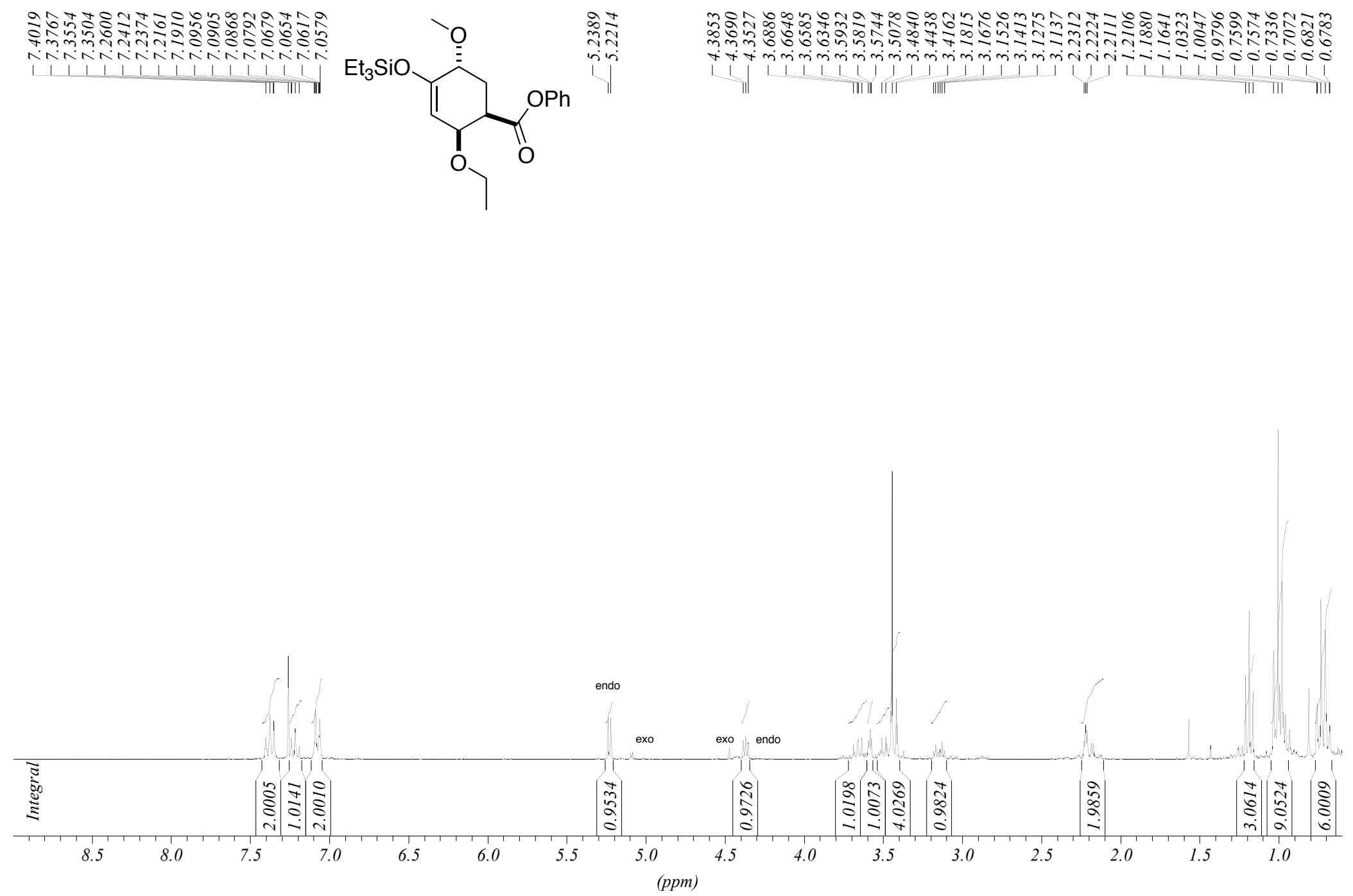
2-Ethoxy-5-methoxy-4-triethylsilyloxycyclohex-3-enecarboxylic acid phenyl ester 29 trans $\left(75 \mathrm{MHz}, \mathrm{CDCl}_{3}\right)$

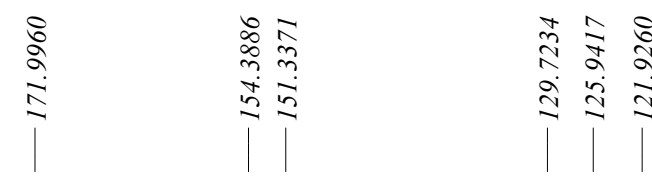

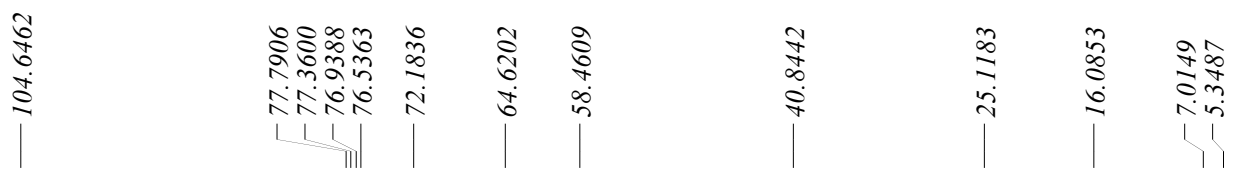

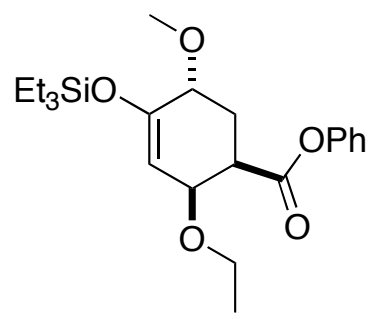

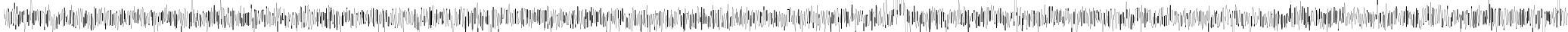

$\begin{array}{lllllllllllllllllll}170 & 160 & 150 & 140 & 130 & 120 & 110 & 100 & 90 & 80 & 70 & 60 & 50 & 40 & 30 & 20 & 10\end{array}$


2-Ethoxy-5-methoxy-4-triethylsilyloxycyclohex-3-enecarboxylic acid phenyl ester 29 cis

$\left(300 \mathrm{MHz}, \mathrm{CDCl}_{3}\right)$

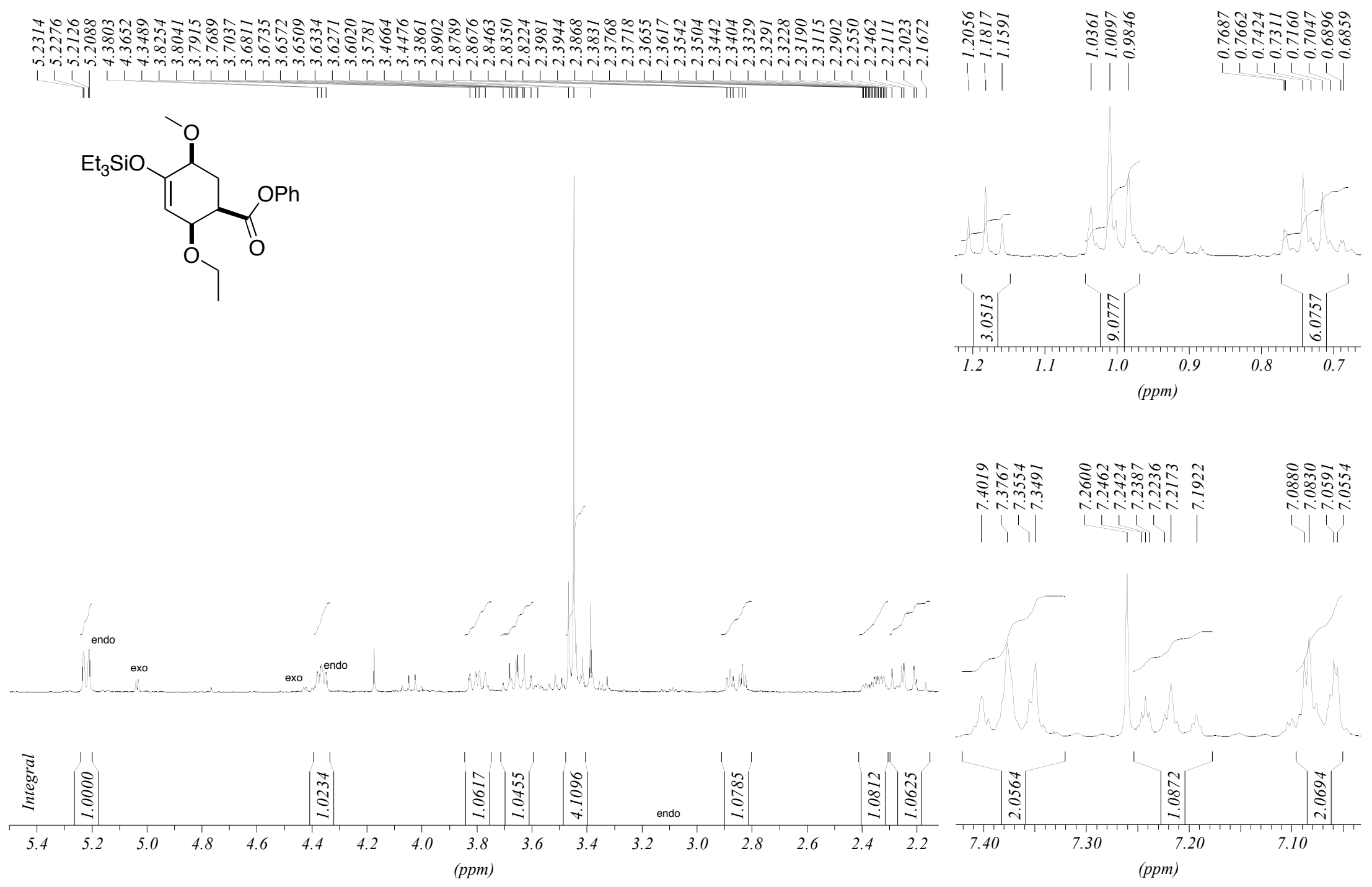


2-Ethoxy-5-methoxy-4-triethylsilyloxycyclohex-3-enecarboxylic acid phenyl ester 29 cis (75 MHz, $\mathrm{CDCl}_{3}$ )

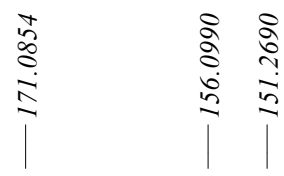
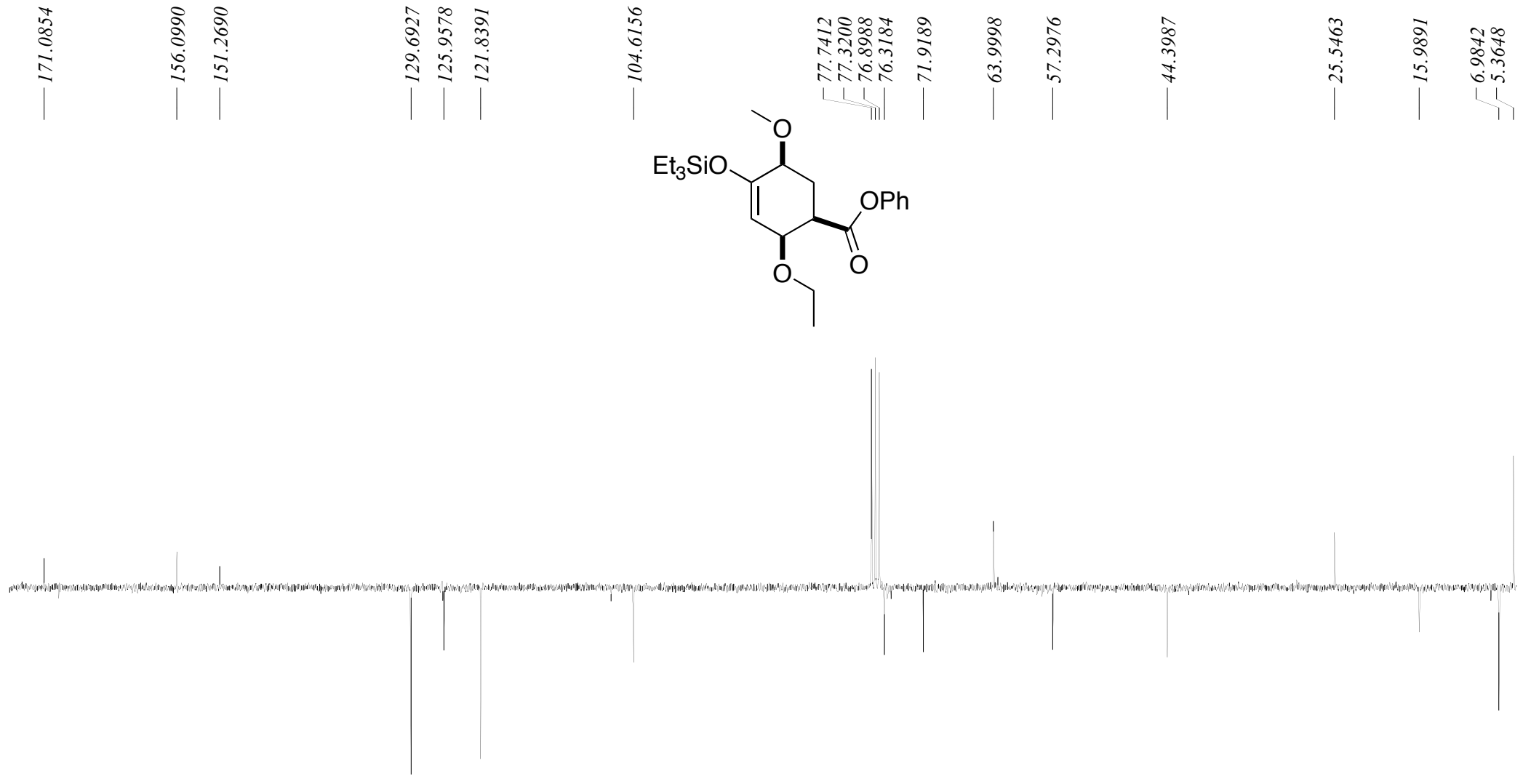

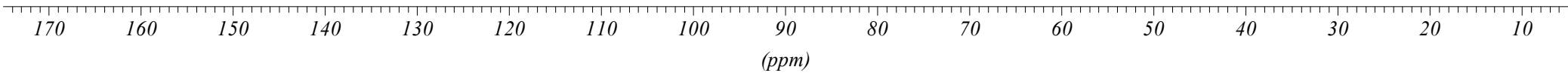


5-tertButyldiphenylsilyloxy-7-ethoxy-4-methoxy-2-methyl-3a,4,7,7a-tetrahydroisoindole-1,3-dione 30 trans $\left(300 \mathrm{MHz}, \mathrm{CDCl}_{3}\right)$
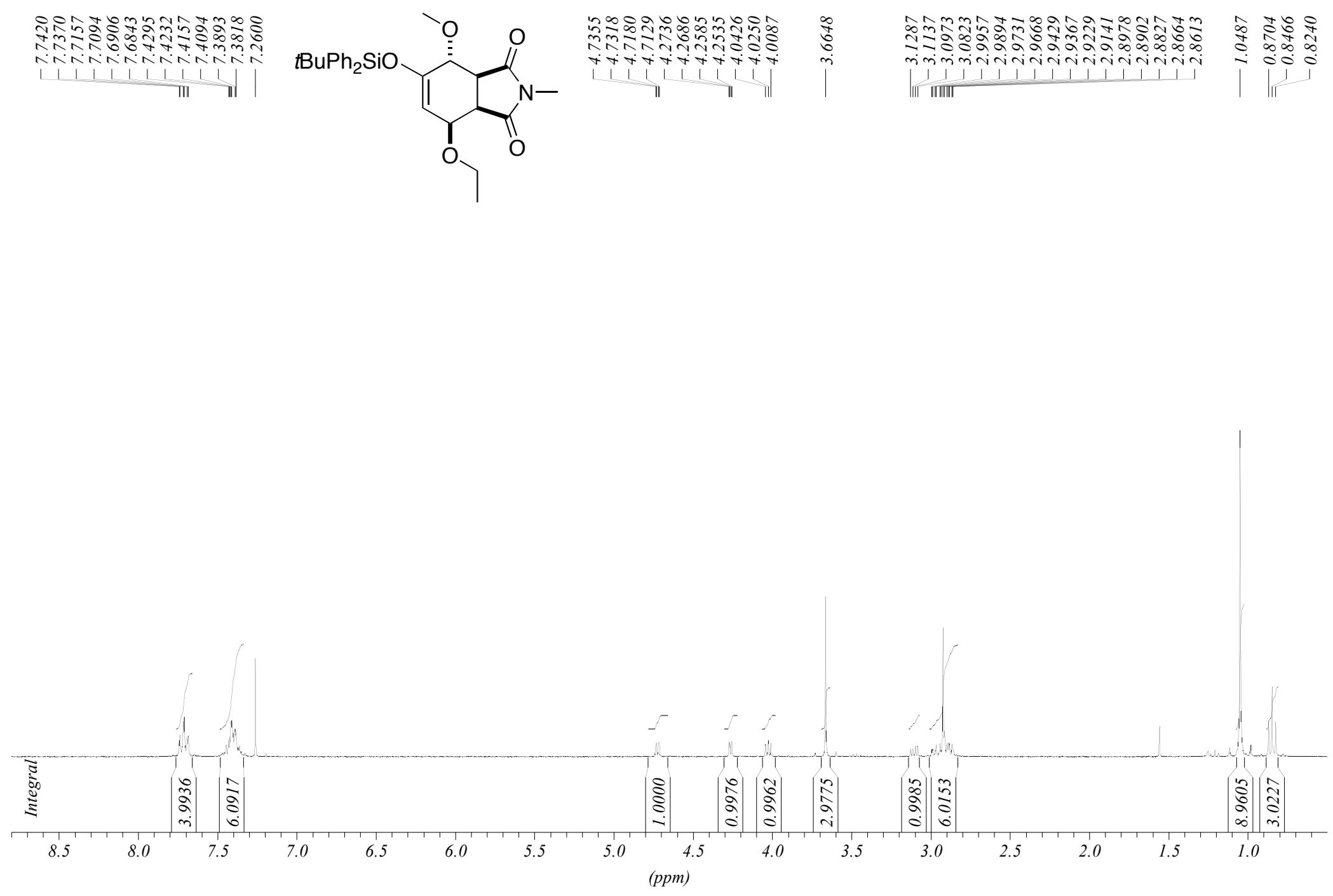
5-tertButyldiphenylsilyloxy-7-ethoxy-4-methoxy-2-methyl-3a,4,7,7a-tetrahydroisoindole-1,3-dione 30 trans (75 MHz, $\mathrm{CDCl}_{3}$ )

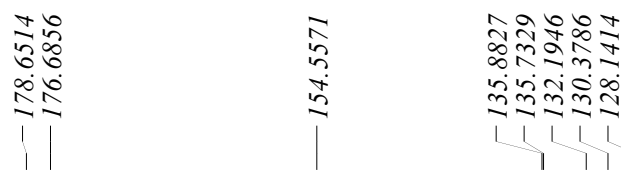

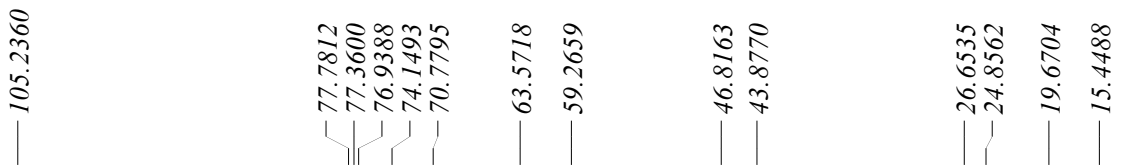

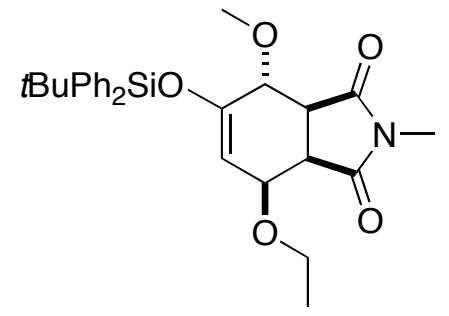

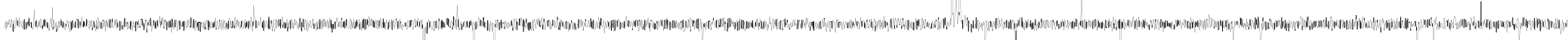


S58

5-tertButyldiphenylsilyloxy-7-ethoxy-4-methoxy-2-methyl-3a,4,7,7a-tetrahydroisoindole-1,3-dione 30 cis (300 MHz, $\mathrm{CDCl}_{3}$ )
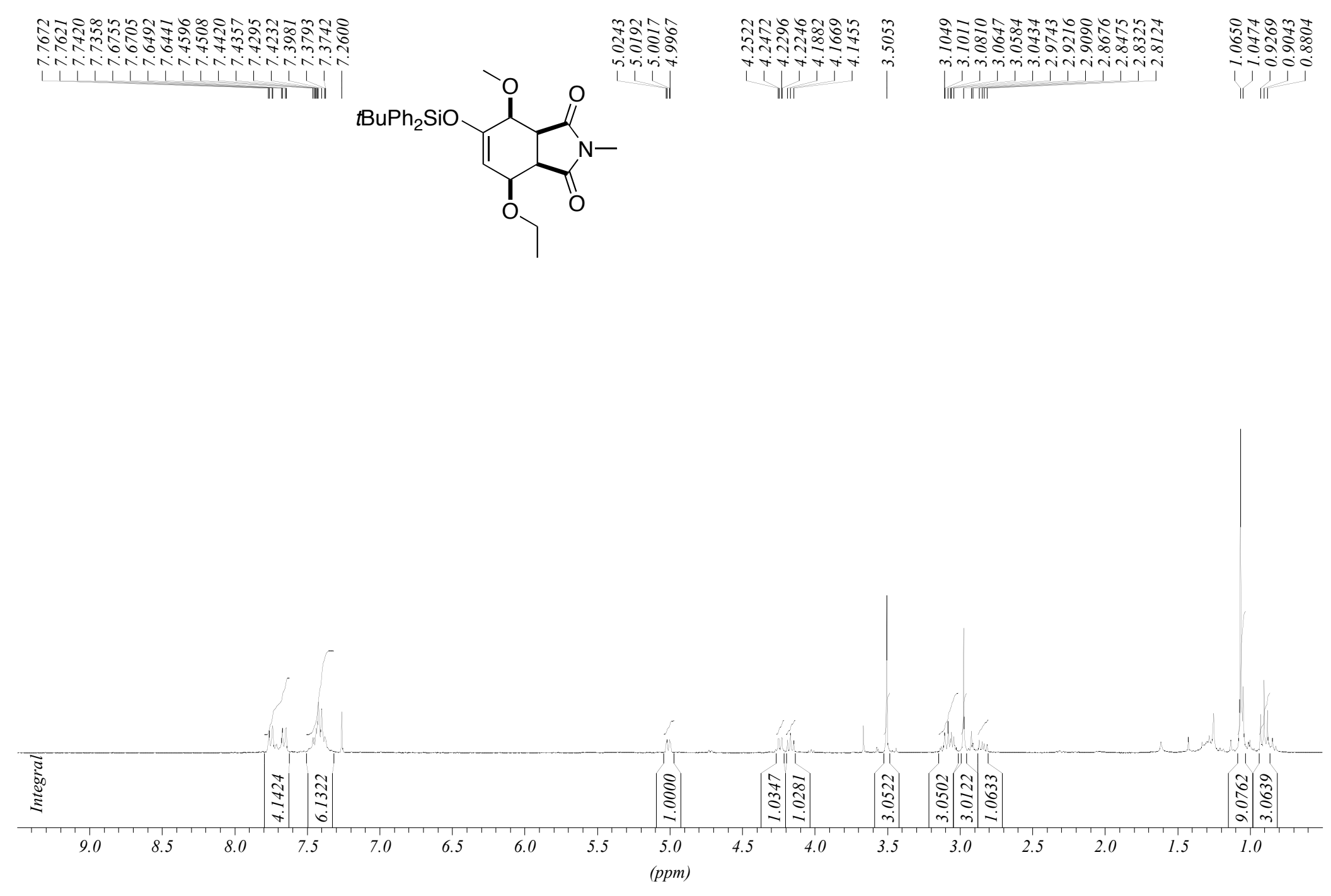
S59

5-tertButyldiphenylsilyloxy-7-ethoxy-4-methoxy-2-methyl-3a,4,7,7a-tetrahydroisoindole-1,3-dione 30 cis (75 MHz, $\mathrm{CDCl}_{3}$ )

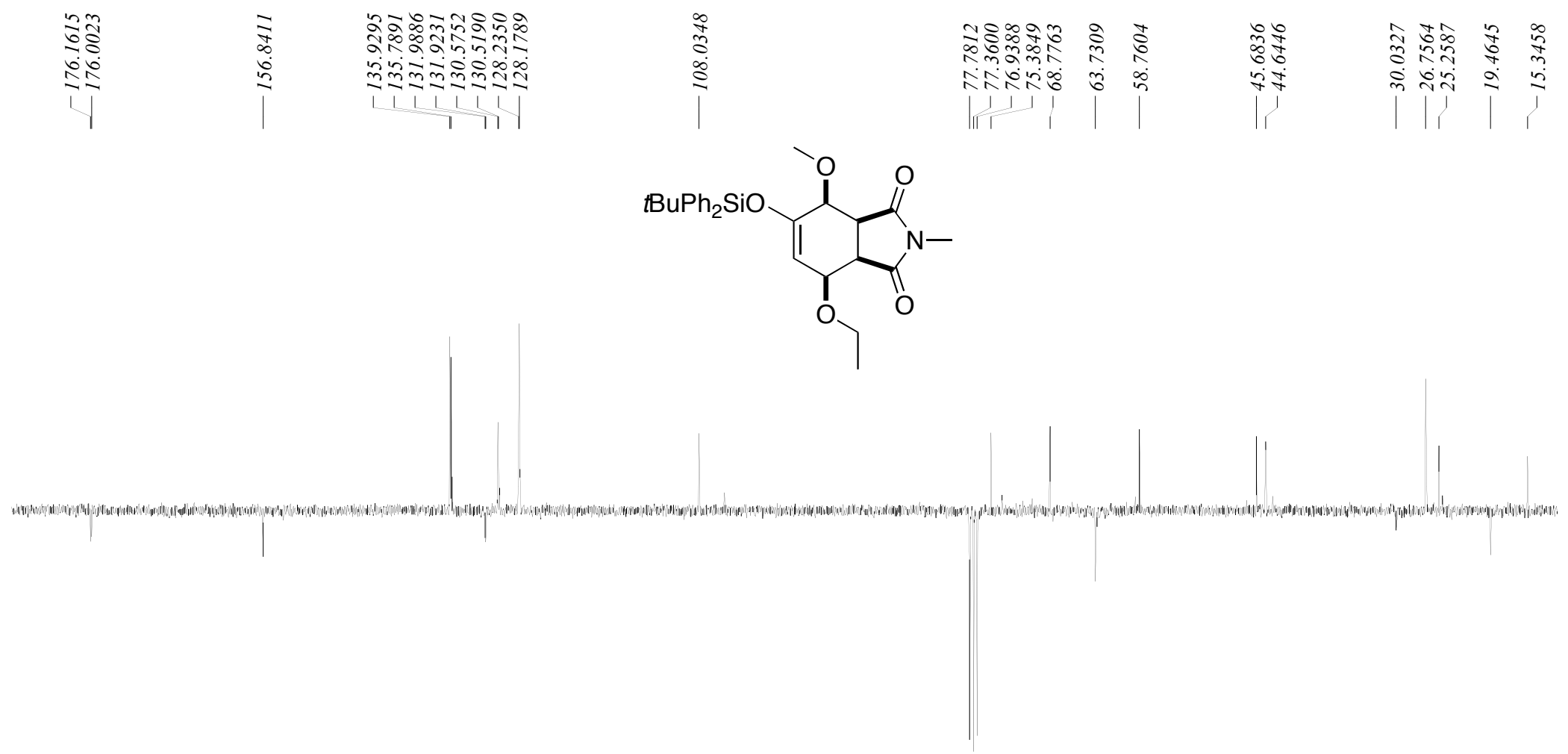

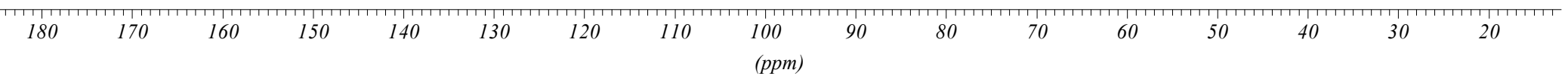


4-tert-Butyldiphenylsilyloxy-2,5-dimethoxycyclohex-3-enecarboxylic acid phenyl ester 31 trans (300 MHz, $\mathrm{CDCl}_{3}$ )
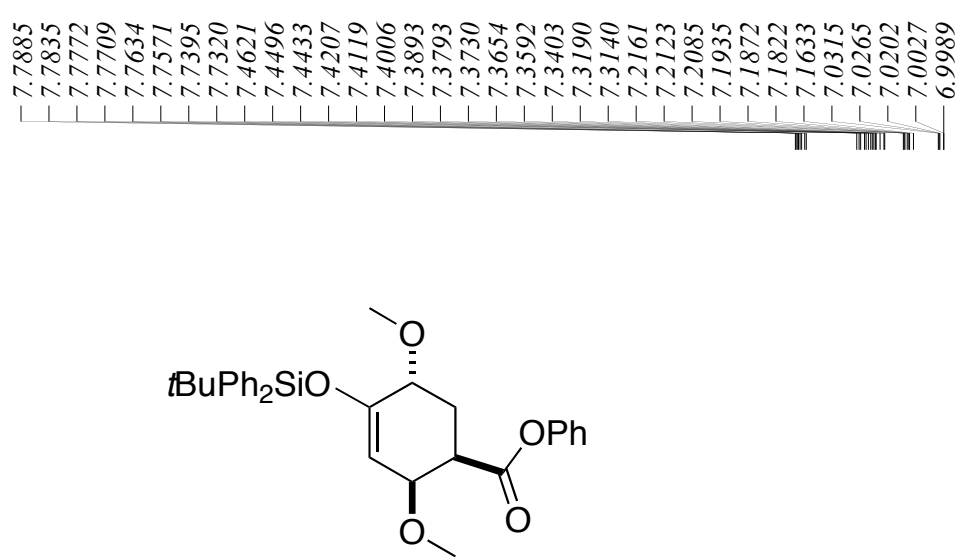

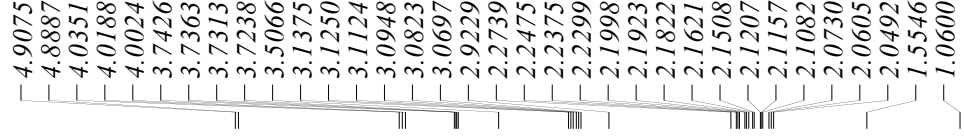

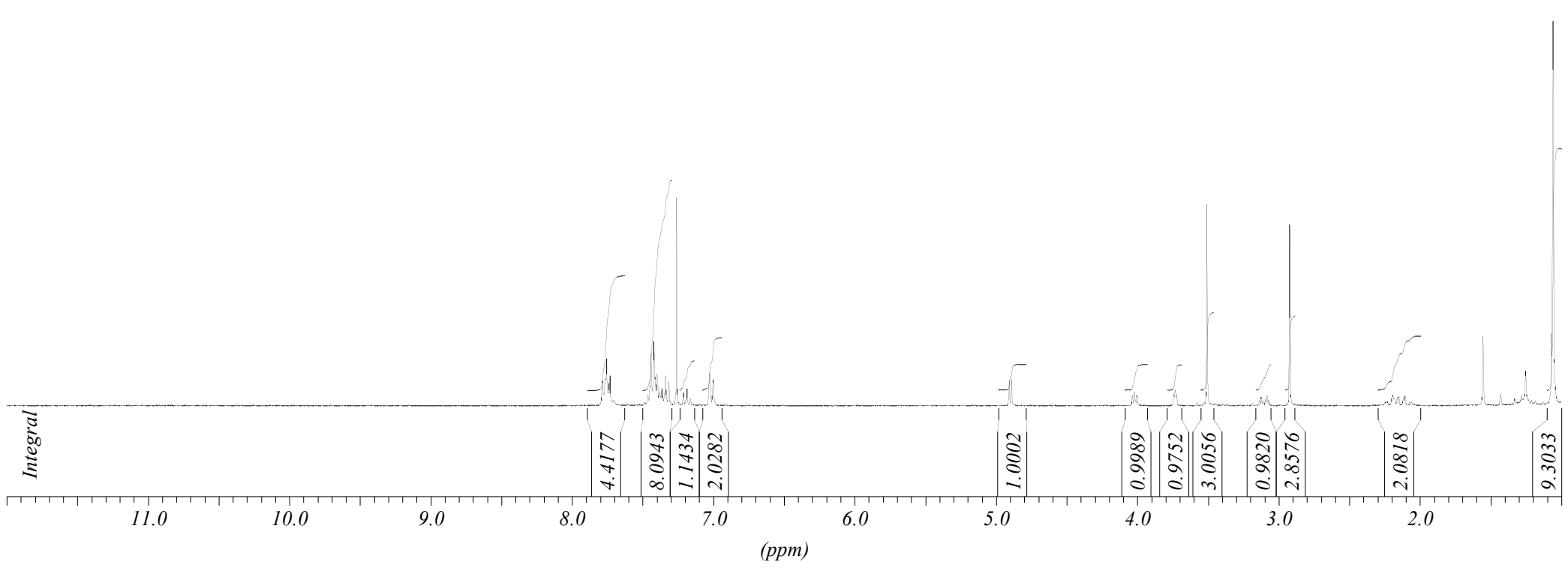


4-tert-Butyldiphenylsilyloxy-2,5-dimethoxycyclohex-3-enecarboxylic acid phenyl ester 31 trans (75 MHz, $\mathrm{CDCl}_{3}$ )
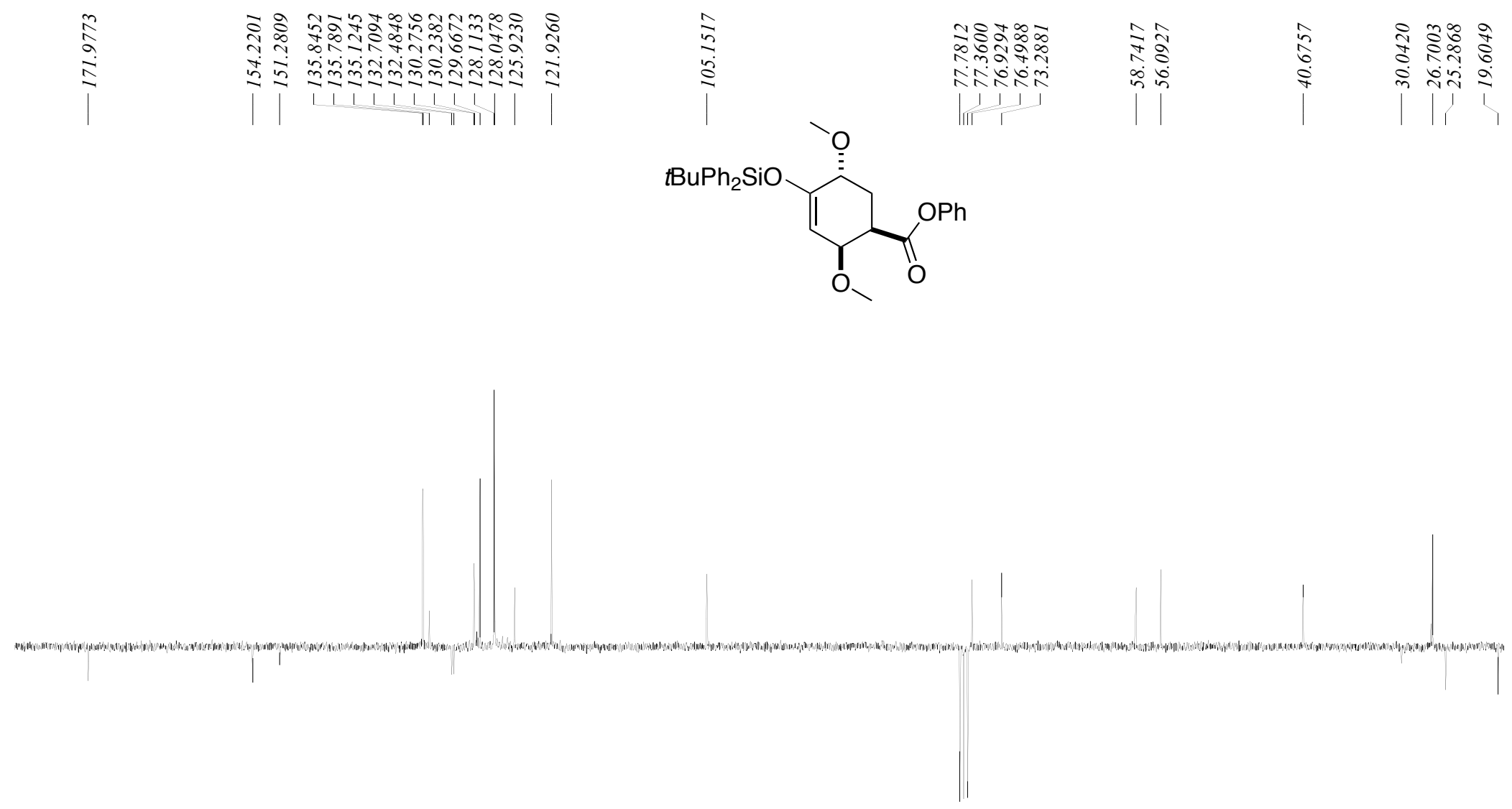

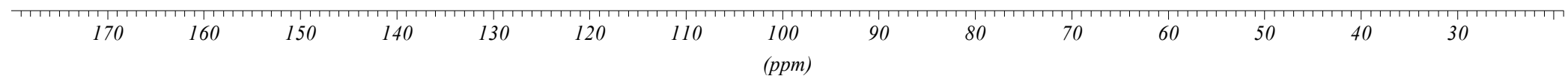

